\title{
Nitrile Biotransformations for Highly Enantioselective Synthesis of Oxiranecarboxamides with a Tertiary and a Quaternary Stereocenters, Efficient Chemoenzymatic Approaches to Enantiopure $\alpha$-Methylated Serine and Isoserine Derivatives
}

\author{
Mei-Xiang Wang, * Gang Deng, De-Xian Wang and Qi-Yu Zheng
}

Laboratory for Chemical Biology, Center for Molecular Science, Institute of Chemistry, Chinese Academy of Sciences, Beijing 100080, China

mxwang@iccas.ac.cn

Table of Contents

1. General Experimental Methods

S1-S2

2. Spectroscopic data of all compounds prepared

S2-S11

3. ${ }^{1} \mathrm{H}$ and ${ }^{13} \mathrm{C}$ NMR spectra of $\mathbf{2}, \mathbf{1 a}, \mathbf{4 a}, \mathbf{4 e}, \mathbf{5 a}, \mathbf{6 - 1 5}, 17$.

S12-S60

4. HPLC analysis of products.

S61-S62

\section{General Experimental Methods}

Both melting points and boiling points are uncorrected. Elemental analyses were performed at the Analytical Laboratory of the Institute. The starting racemic nitriles 1, 6 and $\mathbf{7}$ were 
prepared following a literature method, ${ }^{1}$ and trans- and cis-isomers were separated using column chromatography. The racemic amides were prepared from chemical hydrolysis of nitriles. $^{2}$ The enantiomeric excess values of all compounds were obtained from HPLC analyses.

References

1. Svoboda, J.; Kocfeldová, Z.; Paleček, J. Coll. Czech. Chem. Commun. 1988, 53, 822.

2. Cativela, C.; Diaz-de-Villegas, M. D.; Galvez, J. A. Tetrahedron: Asymmetry 1994, 5, 261.

\section{Spectroscopic data of all compounds prepared}

2R,3S-(-)-2-Methyl-3-phenyl-oxiranecarboxamide (2a): Biotransformation of racemic nitrile 1a for 7.5h gave 2R,3S-(-)-2-methyl-3-phenyl-oxiranecarboxamide 2a: yield 45\%; mp 141-142 $\square ;[\alpha]^{25}-13.6^{\circ}$ (c 3.0, $\mathrm{CH}_{3} \mathrm{OH}$ ); ee>99.5\% (chiral HPLC analysis); ${ }^{1} \mathrm{H}$ NMR (300MHz, $\left.\mathrm{CDCl}_{3}, \mathrm{TMS}\right) \delta$ 7.29-7.42 (m, 5H), 6.45 (s, br, 1H), 5.40 (s, br, 1H), $4.14(\mathrm{~s}, 1 \mathrm{H})$, $1.34(\mathrm{~s}, 3 \mathrm{H}) ;{ }^{13} \mathrm{C} \mathrm{NMR}\left(\mathrm{CDCl}_{3}\right) \delta 174.1,133.5,128.4,128.3,126.6,63.5,62.2,11.9 ; \mathrm{IR}$

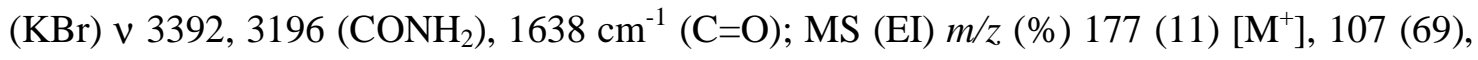
106 (100). Anal. Calcd for $\mathrm{C}_{10} \mathrm{H}_{11} \mathrm{NO}_{2}$ : C, 67.78; H, 6.26; N, 7.90. Found: C, 67.87; H, 6.17; N, 7.81. Kinetic resolution of racemic amide 2a yielded $2 R, 3 S$-(-)-2-methyl-3-phenyl-oxiranecarboxamide $\mathbf{2 a}$ in $44.5 \%$ yield with $81 \%$ ee. When acetone $(2.5 \mathrm{ml})$ was used as an additive, $2 R, 3 S$-(-)-2a was obtained in $41 \%$ yield with $>99.5 \%$ ee.

When the biotransformation of racemic nitrile 1a was quenched in $5 \mathrm{~min}, \mathbf{2 a}(50 \%$, ee $=$ $8 \%)$ was obtained. Racemic nitrile 1a was also recovered: $36 \%[\alpha]_{\mathrm{D}}^{25} 0^{\circ} ;{ }^{1} \mathrm{H}$ NMR $(300 \mathrm{MHz}$, $\left.\mathrm{CDCl}_{3}, \mathrm{TMS}\right) \delta$ 7.25-7.38 (m, 5H), $4.44(\mathrm{~s}, 1 \mathrm{H}), 1.33(\mathrm{~s}, 3 \mathrm{H}) ;{ }^{13} \mathrm{C} \mathrm{NMR}\left(\mathrm{CDCl}_{3}\right) \delta 131.5$, 
129.2, 128.6, 126.8, 119.3, 62.8, 50.1, 15.2; IR (KBr) v 2983, 2242, 1606, 1497, 1453, 1386, 1214, $754 \mathrm{~cm}^{-1}$; MS (EI) m/z (\%) 159 (34) [M+], 131 (39), 116 (41), 90 (100), 89 (51), 77 (14). Anal. Calcd for $\mathrm{C}_{10} \mathrm{H}_{9} \mathrm{NO}$ : C, 75.45; H, 5.70; N, 8.80. Found: C, 75.40; H, 5.68; N, 8.84.

In a multigram-scale biotransformation, racemic substrate 1a $(13.1 \mathrm{mmol}, 2.475 \mathrm{~g})$, which was added portion-wise during 4 days, was incubated with Rhodococcus sp. AJ270 (4 g wet weight cells) for 4 days to give, after workup, intact nitrile 1a (16\%, 0.394g) and enantiopure 2R,3S-(-)-2a (1.058g, 46\%) (based on consumed nitrile).

Incubation of a mixture of racemic trans-2-methyl-3-phenyl-oxiranecarbonitrile $\mathbf{1 a}$ and cis-2-methyl-3-phenyl-oxiranecarbonitrile $4 \mathbf{a}(2 \mathrm{mmol})$ with microbial cells for $5 \mathrm{~h}$ gave, after work-up, 2R,3S-(-)-2-methyl-3-phenyl-oxiranecarboxamide $2 \mathbf{a}$ in $23 \%$ yield with ee $>99.5 \%$ and racemic cis-2-methyl-3-phenyl-oxiranecarbonitrile $4 \mathbf{a}$ in $48 \%$ yield.

2R,3S-(-)-3-(4-Fluorophenyl)-2-methyl-oxiranecarboxamide (2b): 10h; yield 31\%; mp 170.5-172 $\square ;[\alpha]^{25}-6.5^{\circ}\left(c\right.$ 5.0, $\left.\mathrm{CH}_{3} \mathrm{OH}\right)$; ee>99.5\% (chiral HPLC analysis); ${ }^{1} \mathrm{H}$ NMR (300MHz, $\left.\mathrm{CDCl}_{3}, \mathrm{TMS}\right) \delta$ 7.06-7.33 (m, 5H), 6.46 (s, br, 1H), $5.62(\mathrm{~s}, \mathrm{br}, 1 \mathrm{H}), 4.13(\mathrm{~s}, 1 \mathrm{H})$, $1.35(\mathrm{~s}, 3 \mathrm{H}) ;{ }^{13} \mathrm{C} \mathrm{NMR}\left(\mathrm{CDCl}_{3}\right) \delta 174.0,164.4,161.1,129.3,128.4,128.3,115.6,115.3,63.0$, 62.2, 11.9; IR (KBr) v 3387, $3194\left(\mathrm{CONH}_{2}\right), 1640 \mathrm{~cm}^{-1}(\mathrm{C}=\mathrm{O})$; MS (EI) $\mathrm{m} / z(\%) 195$ (10) $\left[\mathrm{M}^{+}\right], 178(9), 161$ (100), 134 (62), 133 (83), 124 (62), 109 (74). Anal. Calcd for $\mathrm{C}_{10} \mathrm{H}_{10} \mathrm{FNO}_{2}$ : C, 61.53; H, 5.16; N, 7.18. Found: C, 61.49; H, 5.16; N, 7.21.

2R,3S-(-)-3-(4-Chlorophenyl)-2-methyl-oxiranecarboxamide (2c): 7.5h; yield 49\%; mp 193.5-195 $\square ;[\alpha]^{25}{ }_{D}-16^{\circ}\left(c\right.$ 3.0, $\left.\mathrm{CH}_{3} \mathrm{OH}\right)$; ee $>99.5 \%$ (chiral HPLC analysis); ${ }^{1} \mathrm{H}$ NMR (300MHz, $\left.\mathrm{CDCl}_{3}, \mathrm{TMS}\right) \delta 7.35(\mathrm{~d}, J=8.34 \mathrm{~Hz}, 2 \mathrm{H}), 7.23(\mathrm{~d}, J=8.25 \mathrm{~Hz}, 2 \mathrm{H}), 6.40$ (s, br, 1H), $5.49(\mathrm{~s}, \mathrm{br}, 1 \mathrm{H}), 4.09(\mathrm{~s}, 1 \mathrm{H}), 1.31(\mathrm{~s}, 3 \mathrm{H}) ;{ }^{13} \mathrm{C} \mathrm{NMR}\left(\mathrm{CDCl}_{3}\right) \delta$ 173.7, 134.4, 132.0, 128.6, 128.0, 62.9, 62.2, 11.9; IR (KBr) v 3384, $3201\left(\mathrm{CONH}_{2}\right), 1660 \mathrm{~cm}^{-1}(\mathrm{C}=\mathrm{O})$; MS (EI) 
$m / z(\%) 213(5), 211(15)\left[\mathrm{M}^{+}\right], 179$ (34), 177 (98), 142 (92), 141 (73), 140 (95), 127 (21), 125 (60), 115 (100), 89 (42). Anal. Calcd for $\mathrm{C}_{10} \mathrm{H}_{10} \mathrm{ClNO}_{2}$ : C, 56.75; H, 4.76; N, 6.62. Found: C, 56.52; H, 4.76; N, 6.50.

2R,3S-(-)-3-(3-Chlorophenyl)-2-methyl-oxiranecarboxamide (2d): 6d; yield 52\%; mp 143-145 $\square ;[\alpha]^{25}{ }_{\mathrm{D}}-4.5^{\circ}\left(c\right.$ 6.0, $\left.\mathrm{CH}_{3} \mathrm{OH}\right)$; ee 41\% (chiral HPLC analysis); ${ }^{1} \mathrm{H}$ NMR (300MHz, $\left.\mathrm{CDCl}_{3}, \mathrm{TMS}\right) \delta$ 7.21-7.35 (m, 4H), $6.43(\mathrm{~s}, \mathrm{br}, 1 \mathrm{H}), 5.44(\mathrm{~s}, \mathrm{br}, 1 \mathrm{H}), 4.12(\mathrm{~s}, 1 \mathrm{H}), 1.36(\mathrm{~s}, 3 \mathrm{H})$; ${ }^{13} \mathrm{C} \mathrm{NMR}\left(\mathrm{CDCl}_{3}\right) \delta 173.6,135.6,134.5,129.7,128.6,126.7,124.8,62.7,62.2,12.0 ; \mathrm{IR}$

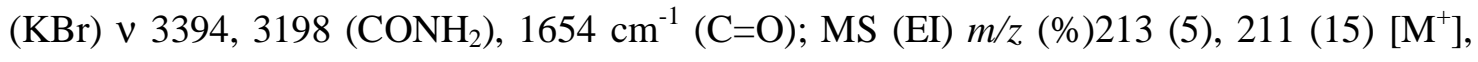
179 (22), 177 (78), 142 (93), 141 (54), 140 (72), 115 (100). Anal. Calcd for $\mathrm{C}_{10} \mathrm{H}_{10} \mathrm{ClNO}_{2}$ : C, 56.75; H, 4.76; N, 6.62. Found: C, 56.74; H, 4.75; N, 6.51.

trans-( \pm )-3-(2-Chlorophenyl)-2-methyl-oxiranecarboxamide (2e): A mixture of racemic trans- and cis-nitrile isomers $1 \mathbf{e}$ and $\mathbf{4 e}$ was incubated with biocatalyst for $7 \mathrm{~d}$ to give racemic trans-(土)-3-(2-Chlorophenyl)-2-methyl-oxiranecarboxamide 2e: yield 21\%; mp 162-163 $\square$; $[\alpha]^{25}{ }_{\mathrm{D}}^{\circ} 0^{\circ}{ }^{1} \mathrm{H}$ NMR $\left(300 \mathrm{MHz}, \mathrm{CDCl}_{3}, \mathrm{TMS}\right) \delta 7.31-7.42(\mathrm{~m}, 4 \mathrm{H}), 6.42(\mathrm{~s}, \mathrm{br}, 1 \mathrm{H}), 5.62(\mathrm{~s}, \mathrm{br}$, 1H), $4.26(\mathrm{~s}, 1 \mathrm{H}), 1.30(\mathrm{~s}, 3 \mathrm{H}) ;{ }^{13} \mathrm{C} \mathrm{NMR}\left(\mathrm{CDCl}_{3}\right) \delta 173.4,133.3,132.0,129.5,129.3,128.0$, 126.6, 62.1, 62.0, 12.2; IR (KBr) v 3423, $3193\left(\mathrm{CONH}_{2}\right), 1660 \mathrm{~cm}^{-1}(\mathrm{C}=\mathrm{O}) ; \mathrm{MS}(\mathrm{EI}) \mathrm{m} / z(\%)$ 213 (4), $211(13)\left[\mathrm{M}^{+}\right], 179$ (13), 177 (50), 160 (49), 142 (66), 141 (74), 140 (100), 115 (67), 89 (47). Anal. Calcd for $\mathrm{C}_{10} \mathrm{H}_{10} \mathrm{ClNO}_{2}$ : C, 56.75; H, 4.76; N, 6.62. Found: C, 56.36; H, 4.77; $\mathrm{N}, 6.51$. cis-( \pm )-3-(2-Chlorophenyl)-2-methyl-oxiranecarbonitrile 4e was recovered: yield $45 \% ;[\alpha]^{25}{ }_{\mathrm{D}}^{\circ} ;{ }^{1} \mathrm{H}$ NMR $\left(300 \mathrm{MHz}, \mathrm{CDCl}_{3}, \mathrm{TMS}\right) \delta 7.31-7.39(\mathrm{~m}, 4 \mathrm{H}), 4.23(\mathrm{~s}, 1 \mathrm{H}), 1.83(\mathrm{~s}$, $3 \mathrm{H}) ;{ }^{13} \mathrm{C} \mathrm{NMR}\left(\mathrm{CDCl}_{3}\right) \delta \quad 133.2,130.8,130.4,129.3,127.2,127.1,116.8,62.0,53.1,20.5$;

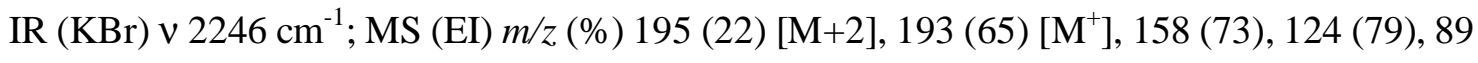


(100). Anal. Calcd for $\mathrm{C}_{10} \mathrm{H}_{8} \mathrm{ClNO}$ : C, 62.03; H, 4.16; N, 7.23. Found: C, 62.10; H, 4.04; N, 7.16 .

2R,3S-(-)-3-(4-Bromophenyl)-2-methyl-oxiranecarboxamide (2f): $8.5 \mathrm{~h}$; yield 48\%; mp 193-194 $\square ;[\alpha]^{25}{ }_{D}-18.2^{\circ}$ (c 3.0, $\mathrm{CH}_{3} \mathrm{OH}$ ); ee $>99.5 \%$ (chiral HPLC analysis); ${ }^{1} \mathrm{H}$ NMR $\left(300 \mathrm{MHz}, \mathrm{CDCl}_{3}, \mathrm{TMS}\right) \delta 7.55(\mathrm{~d}, J=8.27 \mathrm{~Hz}, 2 \mathrm{H}), 7.22(\mathrm{~d}, J=8.37 \mathrm{~Hz}, 2 \mathrm{H}), 6.45(\mathrm{~s}, \mathrm{br}$, 1H), 5.41 (s, br, 1H), $4.12(\mathrm{~s}, 1 \mathrm{H}), 1.35(\mathrm{~s}, 3 \mathrm{H}) ;{ }^{13} \mathrm{C}$ NMR $\left(\mathrm{CDCl}_{3}\right) \delta$ 173.6, 132.4, 131.4, 128.1, 122.3, 62.8, 62.0, 11.7; IR (KBr) v 3383, $3200\left(\mathrm{CONH}_{2}\right), 1659 \mathrm{~cm}^{-1}(\mathrm{C}=\mathrm{O})$; MS (EI) $m / z(\%) 257$ (12), $255(12)\left[\mathrm{M}^{+}\right], 223$ (78), 221 (81), 187 (33), $186(56), 185$ (63), 184 (61), 115 (100), 89 (46). Anal. Calcd for $\mathrm{C}_{10} \mathrm{H}_{10} \mathrm{BrNO}_{2}$ : C, 46.90; H, 3.94; N, 5.47. Found: C, 47.02; H, 4.01; N, 5.36.

2R,3S-(-)-2-Methyl-3-(4-methylphenyl)glycidamide (2g): 11.5h; yield 31\%; mp 168-170 $\square ;[\alpha]^{25}-5.9^{\circ}\left(c\right.$ 5.0, $\left.\mathrm{CH}_{3} \mathrm{OH}\right)$; ee>99.5\% (chiral HPLC analysis); ${ }^{1} \mathrm{H}$ NMR $(300 \mathrm{MHz}$, $\left.\mathrm{CDCl}_{3}, \mathrm{TMS}\right) \delta 7.18(\mathrm{~s}, 4 \mathrm{H}), 6.44(\mathrm{~s}, \mathrm{br}, 1 \mathrm{H}), 5.59$ (s, br, 1H), 4.09 (s, 1H), 2.35 (s, 3H), 1.32 $(\mathrm{s}, 3 \mathrm{H}) ;{ }^{13} \mathrm{C} \mathrm{NMR}\left(\mathrm{CDCl}_{3}\right) \delta 174.1,138.0,130.2,128.8,126.4,63.4,62.0,21.0,11.7$; IR $(\mathrm{KBr}) \vee 3384,3189\left(\mathrm{CONH}_{2}\right), 1661,1638 \mathrm{~cm}^{-1}(\mathrm{C}=\mathrm{O})$; MS (EI) $\mathrm{m} / z(\%) 191(24)\left[\mathrm{M}^{+}\right], 157$ (94), 156 (53), 120 (58), 119 (55), 105 (100), 91 (39). Anal. Calcd for $\mathrm{C}_{11} \mathrm{H}_{12} \mathrm{NO}_{2}$ : C, 69.09; H, 6.85; N, 7.32. Found: C, 68.84; H, 6.84; N, 7.30.

trans-( \pm )-2-Methyl-3-(2-methylphenyl)-oxiranecarboxamide (2h): 7d; yield 44\%; mp 165-166 $\square ;[\alpha]^{25}{ }_{\mathrm{D}}^{\circ}$; ${ }^{1} \mathrm{H}$ NMR $\left(300 \mathrm{MHz}, \mathrm{CDCl}_{3}, \mathrm{TMS}\right) \delta$ 7.18-7.26 (m, 5H), 6.49 (s, br, 1H), $5.84(\mathrm{~s}, \mathrm{br}, 1 \mathrm{H}), 4.10(\mathrm{~s}, 1 \mathrm{H}), 2.32(\mathrm{~s}, 3 \mathrm{H}), 1.26(\mathrm{~s}, 3 \mathrm{H}) ;{ }^{13} \mathrm{C} \mathrm{NMR}\left(\mathrm{CDCl}_{3}\right) \delta 173.8$, $136.0,132.1,129.9,128.2,125.9,125.8,62.7,61.8,18.7,12.2$; IR $(\mathrm{KBr}) \vee 3410,3183$

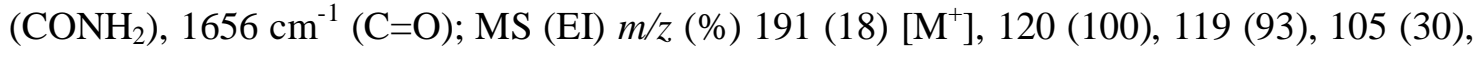


91 (62). Anal. Calcd for $\mathrm{C}_{10} \mathrm{H}_{13} \mathrm{NO}_{2}$ : C, 69.09; H, 6.85; N, 7.32. Found: C, 68.90; H, 6.84; N, 7.36 .

2R,3S-(-)-2-Methyl-3-(2,3-methylenedioxyphenyl)-oxiranecarboxamide (2i): 1d; yield 32\%; mp 123-125 $\square$; $[\alpha]^{25}-9.3^{\circ}\left(c\right.$ 6.0, $\left.\mathrm{CH}_{3} \mathrm{OH}\right)$; ee 50\% (chiral HPLC analysis); ${ }^{1} \mathrm{H}$ NMR (300MHz, $\left.\mathrm{CDCl}_{3}, \mathrm{TMS}\right) \delta$ 6.76-6.83 (m, 3H), 6.41 (s, br, 1H), $5.96(\mathrm{~s}, 2 \mathrm{H}), 5.61$ (s, br, 1H), $4.04(\mathrm{~s}, 1 \mathrm{H}), 1.34(\mathrm{~s}, 3 \mathrm{H}) ;{ }^{13} \mathrm{C}$ NMR $\left(\mathrm{CDCl}_{3}\right) \delta 174.2,147.7,127.3,120.3,108.3,107.0$, 101.3, 63.4, 62.3, 11.8; IR $(\mathrm{KBr}) \vee 3417,3369,3188\left(\mathrm{CONH}_{2}\right), 1657 \mathrm{~cm}^{-1}(\mathrm{C}=\mathrm{O})$; MS $(\mathrm{EI})$ $m / z(\%) 221(13)\left[\mathrm{M}^{+}\right], 187$ (35), $186(20), 178$ (21), 150 (42), 149 (25), 135 (100), 85 (34), 83 (52). Anal. Calcd for $\mathrm{C}_{11} \mathrm{H}_{11} \mathrm{NO}_{4}$ : C, 59.73; H, 5.01; N, 6.33. Found: C, 59.44; H, 5.01; N, 6.33 .

cis-(-)-2-Methyl-3-phenyl-oxiranecarboxamide cis-2-methyl-3-phenyl-oxiranecarboxamide

cis-2-methyl-3-phenyl-oxiranecarbonitrile

cis-(-)-2-methyl-3-phenyl-oxiranecarboxamide (4a): Biotransformation of racemic 4a $\quad$ in $\quad 7 d \quad$ afforded 5a and intact racemic $-10.6^{\circ}$ (c 2.9, $\left.\mathrm{CH}_{3} \mathrm{OH}\right)$; ee $28 \%$ (chiral HPLC analysis); ${ }^{1} \mathrm{H}$ NMR $\left(300 \mathrm{MHz}, \mathrm{CDCl}_{3}, \mathrm{TMS}\right) \delta$ $7.31(\mathrm{~s}, 5 \mathrm{H}), 5.96(\mathrm{~s}, \mathrm{br}, 1 \mathrm{H}), 5.17(\mathrm{~s}, \mathrm{br}, 1 \mathrm{H}), 4.13(\mathrm{~s}, 1 \mathrm{H}), 1.74(\mathrm{~s}, 3 \mathrm{H}) ;{ }^{13} \mathrm{C} \mathrm{NMR}\left(\mathrm{CDCl}_{3}\right) \delta$ 171.1, 133.7, 128.3, 126.3, 64.8, 63.3, 18.9; IR (KBr) v 3390, $3202\left(\mathrm{CONH}_{2}\right), 1655 \mathrm{~cm}^{-1}$ $(\mathrm{C}=\mathrm{O}) ; \mathrm{MS}(\mathrm{EI}) \mathrm{m} / z(\%) 177$ (25) [M+1, 107 (62), 106 (100), 105 (41), 90 (39), 89 (35). Anal. Calcd for $\mathrm{C}_{10} \mathrm{H}_{11} \mathrm{NO}_{2}$ : C, 67.78; H, 6.26; N, 7.90. Found: C, 67.69; H, 6.51; N, 7.77. ( \pm )-4a: yield 66\%; $[\alpha]^{25}{ }_{\mathrm{D}} 0^{\circ} ;{ }^{1} \mathrm{H}$ NMR $\left(300 \mathrm{MHz}, \mathrm{CDCl}_{3}\right.$, TMS) $\delta 7.35(\mathrm{~s}, 5 \mathrm{H}), 3.92(\mathrm{~s}, 1 \mathrm{H}), 1.64(\mathrm{~s}$, $3 \mathrm{H}) ;{ }^{13} \mathrm{C} \mathrm{NMR}\left(\mathrm{CDCl}_{3}\right) \delta 132.3,129.5,128.6,126.4,117.3,64.1,53.6,20.8$; IR $(\mathrm{KBr}) \vee 2244$ 
$\mathrm{cm}^{-1}$; MS (EI) m/z (\%) 159 (48) [M+1, 131 (34), 116 (51), 90 (100), 89 (52), 77 (15). Anal.

Calcd for $\mathrm{C}_{10} \mathrm{H}_{9} \mathrm{NO}: \mathrm{C}, 75.45 ; \mathrm{H}, 5.70 ; \mathrm{N}, 8.80$. Found: C, 75.21; H, 5.69; N, 8.93.

trans-(-)-2,3-Dimethyl-3-phenyl-oxiranecarbonitrile nitrile (6) and trans-(+)-2,3-dimethyl-3-phenyl-oxiranecarboxamide (8): Biotransformation of racemic trans-2,3-dimethyl-3-phenyl-oxiranecarbonitrile $\quad 6 \quad$ in $8 \quad \min$ yielded trans-(-)-2,3-dimethyl-3-phenyl-oxiranecarbonitrile (-)-6 in $48 \%$ yield and trans-(+)-2,3-dimethyl-3-phenyl-oxiranecarboxamide (+)-8 in $44 \%$ yield. A week's incubation afforded (+)-8 in 64\% yield. (-)-6: $8 \mathrm{~min}$; yield 48.2\%; $[\alpha]^{25}-9.6^{\circ}$ (c 8.0, Acetone); ee 14\% (chiral HPLC analysis); ${ }^{1} \mathrm{H} \mathrm{NMR}\left(300 \mathrm{MHz}, \mathrm{CDCl}_{3}\right.$, TMS) $\delta$ 7.27-7.38 (m, 5H), $1.91(\mathrm{~s}, 3 \mathrm{H}), 1.28(\mathrm{~s}, 3 \mathrm{H}) ;{ }^{13} \mathrm{C} \mathrm{NMR}\left(\mathrm{CDCl}_{3}\right) \delta 136.5,128.6,128.5,126.3,118.6,67.4$, 55.0, 22.7, 17.7; IR (KBr) v $2241 \mathrm{~cm}^{-1}(\mathrm{CN})$; MS (EI) $\mathrm{m} / z(\%) 173(40)\left[\mathrm{M}^{+}\right], 131$ (53), 104 (100), 103 (49), 78 (38). Anal. Calcd for $\mathrm{C}_{11} \mathrm{H}_{11} \mathrm{NO}$ : C, 76.28; H, 6.40; N, 8.09. Found: C, 76.07; H, 6.24; N, 8.33. (+)-8: 7d; yield 64\%; mp 165-168ロ; $[\alpha]^{25}{ }_{\mathrm{D}}+8.1^{\circ}\left(\mathrm{c} 0.8, \mathrm{CHCl}_{3}\right)$; ee $35 \%$ (chiral HPLC analysis); ${ }^{1} \mathrm{H}$ NMR $\left(300 \mathrm{MHz}, \mathrm{CDCl}_{3}, \mathrm{TMS}\right) \delta 7.28-7.40(\mathrm{~m}, 5 \mathrm{H}), 6.45$ (s, br, $1 \mathrm{H}), 6.01(\mathrm{~s}, \mathrm{br}, 1 \mathrm{H}), 1.70(\mathrm{~s}, 3 \mathrm{H}), 1.21(\mathrm{~s}, 3 \mathrm{H}) ;{ }^{13} \mathrm{C} \mathrm{NMR}\left(\mathrm{CDCl}_{3}\right) \delta 173.3,139.1,128.3$, 127.7, 126.1, 67.9, 67.0, 20.6, 15.9; IR (KBr) v 3379, $3185\left(\mathrm{CONH}_{2}\right), 1657 \mathrm{~cm}^{-1}(\mathrm{C}=\mathrm{O})$; MS (EI) $m / z(\%) 191(5)\left[\mathrm{M}^{+}\right], 146$ (30), 120 (55), 104 (100), 91(18), 78 (27). Anal. Calcd for $\mathrm{C}_{10} \mathrm{H}_{13} \mathrm{NO}_{2}$ : C, 69.09; H, 6.85; N, 7.32. Found: C, 69.06; H, 6.83; N, 7.45.

cis-(+)-2,3-Dimethyl-3-phenyl-oxiranecarbonitrile

(7)

and cis-(-)-2,3-dimethyl-3-phenyl-oxiranecarboxamide (9): Biotransformation of racemic cis-2,3-dimethyl-3-phenyl-oxiranecarbonitrile $\quad 7 \quad(0.5 \quad \mathrm{mmol})$ in 5 days yielded cis-(-)-2,3-dimethyl-3-phenyl-oxiranecarbonitrile $\quad(+)-7 \quad$ in $35 \%$ yield and cis-(-)-2,3-dimethyl-3-phenyl-oxiranecarboxamide (-)-9 in 63\% yield. (+)-7: $[\alpha]^{25}+11.3^{\circ}(c$ 
1.5, acetone); ee 35\% (chiral HPLC analysis); ${ }^{1} \mathrm{H}$ NMR $\left(300 \mathrm{MHz}, \mathrm{CDCl}_{3}, \mathrm{TMS}\right) \delta 7.40-7.42$ (m, 5H), $1.78(\mathrm{~s}, 3 \mathrm{H}), 1.73(\mathrm{~s}, 3 \mathrm{H}) ;{ }^{13} \mathrm{C} \mathrm{NMR}\left(\mathrm{CDCl}_{3}\right) \delta 138.1,128.7,128.5,125.7,118.2$, 66.8, 56.0, 18.9, 17.9; IR (KBr) v $2238 \mathrm{~cm}^{-1}(\mathrm{CN})$; MS (EI) $\mathrm{m} / z(\%) 173(54)\left[\mathrm{M}^{+}\right], 131$ (61), 104 (100), 103 (74), 78 (43). Anal. Calcd for $\mathrm{C}_{11} \mathrm{H}_{11} \mathrm{NO}$ : C, 76.28; H, 6.40; N, 8.09. Found: C,

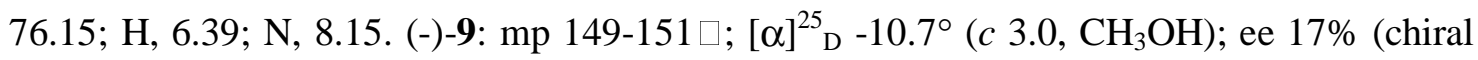
HPLC analysis); ${ }^{1} \mathrm{H}$ NMR (300MHz, $\mathrm{CDCl}_{3}$, TMS) $\delta$ 7.25-7.34 (m,5H), 5.84 (s, br, 1H), 5.18 (s, br, 1H), $1.71(\mathrm{~s}, 3 \mathrm{H}), 1.65(\mathrm{~s}, 3 \mathrm{H}) ;{ }^{13} \mathrm{C} \mathrm{NMR}\left(\mathrm{CDCl}_{3}\right) \delta 172.6,139.5,128.3,127.8,125.9$, 67.9, 65.9, 21.6, 15.4; IR (KBr) v 3428, $3201\left(\mathrm{CONH}_{2}\right), 1661 \mathrm{~cm}^{-1}(\mathrm{C}=\mathrm{O}) ; \mathrm{MS}(\mathrm{EI}) \mathrm{m} / z(\%)$ $191(6)\left[\mathrm{M}^{+}\right], 146$ (50), 131 (28), 103 (100), 77(30). Anal. Calcd for $\mathrm{C}_{10} \mathrm{H}_{13} \mathrm{NO}_{2}: \mathrm{C}, 69.09 ; \mathrm{H}$, 6.85; N, 7.32. Found: C, 69.26; H, 7.17; N, 7.25.

Synthesis of $\boldsymbol{R}$-(+)-2-hydroxy-2-methyl-3-phenylpropionic acid (11). Under hydrogen atmosphere, a mixture of 2R,3S-(-)-2-methyl-3-phenyl-oxiranecarboxamide $2 \mathbf{a}(1 \mathrm{mmol})$, $\mathrm{Pd} / \mathrm{C}(5 \%, 52.3 \mathrm{mg})$ and molecular sieve $(4 \AA, 1 \mathrm{~g})$ in anhydrous THF (10 ml) and methanol (5 $\mathrm{ml}$ ) was stirred at room temperature for $20 \mathrm{~min}$. After filtration with a Celite pad, and removal of the solvents under reduced pressure, the residue was chromatographed on a short silica gel column using a mixture of petroleum ether and ethyl acetate (1:2) as an eluent to give $R$-(+)-2-methyl-2-hydroxy-3-phenylpropionamide 10: yield 99.5\%; $[\alpha]^{25}{ }_{\mathrm{D}}+38^{\circ}$ (c 1.0 , acetone); ee $>99.5 \%$ (chiral HPLC analysis); ${ }^{1} \mathrm{H}$ NMR $\left(300 \mathrm{MHz}, \mathrm{CDCl}_{3}, \mathrm{TMS}\right) \delta$ 7.22-7.35 (m, 5H), $6.56(\mathrm{~s}, \mathrm{br}, 1 \mathrm{H}), 5.70(\mathrm{~s}, \mathrm{br}, 1 \mathrm{H}), 3.28(\mathrm{~d}, 1 \mathrm{H}, J=13.5 \mathrm{~Hz}), 2.84(\mathrm{~d}, 1 \mathrm{H}, J=13.5 \mathrm{~Hz})$, $2.51(\mathrm{~s}, 1 \mathrm{H}), 1.47(\mathrm{~s}, 3 \mathrm{H}) ;{ }^{13} \mathrm{C} \mathrm{NMR}\left(\mathrm{CDCl}_{3}\right) \delta 178.5,135.7,130.4,128.6,127.2,76.0,45.4$, 26.3; IR (KBr) v 3471, 3340, $1669 \mathrm{~cm}^{-1}$; MS (ESI) $\mathrm{m} / \mathrm{z} 180(\mathrm{M}+1)$; Anal. Calcd for $\mathrm{C}_{10} \mathrm{H}_{13} \mathrm{NO}_{2}$ : C, 67.02; H, 7.31; N, 7.82. Found: C, 66.89; H, 7.30; N, 7.93. $R$-(+)-2-Methyl-2-hydroxy-3-phenylpropionamide $\mathbf{1 0}(1 \mathrm{mmol}, 179 \mathrm{mg})$ was refluxed in 
hydrochloric acid $(6 \mathrm{~N}, 29 \mathrm{ml})$ for $3 \mathrm{~h}$ to give, after extraction with ethyl acetate and column chromatography using a silica gel column with a mixture of petroleum ether and ethyl acatate (1:2) as an eluent, $R$-(+)-2-methyl-2-hydroxy-3-phenylpropionic acid as white solid: yield $92 \% ; \mathrm{mp} 112-114 \square ;[\alpha]_{\mathrm{D}}^{25}+17.2^{\circ}$ (c 1.51, dioxane) $\left[\mathrm{lit},{ }^{19} \mathrm{mp} 115-117 \square,[\alpha]^{25}+13.2^{\circ}(\mathrm{c}\right.$ 1.51 , dioxane $)^{26}$; ee $>99.5 \%$ (chiral HPLC analysis); ${ }^{1} \mathrm{H}$ NMR $\left(300 \mathrm{MHz}, \mathrm{CDCl}_{3}, \mathrm{TMS}\right) \delta$ 7.21-7.30 (m, 5H), $3.15(\mathrm{~d}, 1 \mathrm{H}, J=13.5 \mathrm{~Hz}), 2.94(\mathrm{~d}, 1 \mathrm{H}, J=13.5 \mathrm{~Hz}), 1.54(\mathrm{~s}, 3 \mathrm{H}) ;{ }^{13} \mathrm{C}$ $\operatorname{NMR}\left(\mathrm{CDCl}_{3}\right) \delta 180.9,135.3,130.1,128.4,127.2,75.2,46.0,25.7$; IR (KBr) v 3452, 3424, 2611-3029, 1736, 1721, 1712, $1195 \mathrm{~cm}^{-1}$.

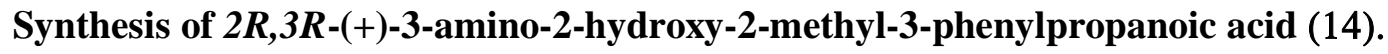

\section{Ring opening reaction of $2 R, 3 S$-(-)-2-methyl-3-phenylglycidamide 2 a with sodium}

azide: A mixture of $2 R, 3 S$-(-)-2-methyl-3-phenyl-oxiranecarboxamide $2 \mathbf{a}$ (1 mmol, ee $>99.5 \%), \mathrm{NaN}_{3}(2.09 \mathrm{mmol})$ and $\mathrm{MgSO}_{4}(1.03 \mathrm{mmol})$ in methanol $(29 \mathrm{ml})$ was refluxed for 24h. After removal of the solvent, the residue was refluxed in ethyl acetate. After filtration and concentration, the organic residue was chromatographed on a silica gel column with a mixture of petroleum ether and ethyl acetate (1:1) as an eluent to give 2R,3R-(-)-3-azido-2-hydroxy-2-methyl-3-phenylpropionamide $\mathbf{1 2}$ as white solid: yield 90\%; mp 180-181 $\square ;[\alpha]^{25}{ }_{\mathrm{D}}-152^{\circ}\left(c\right.$ 1.0, $\left.\mathrm{CH}_{3} \mathrm{OH}\right)$; ee $>99.5 \%$ (chiral HPLC analysis); ${ }^{1} \mathrm{H}$ NMR (300MHz, $\left.\mathrm{CDCl}_{3}, \mathrm{TMS}\right) \delta$ 7.34-7.41 (m, 5H), 6.32 (s, br, 1H), 5.11 (s, br, 1H), $4.94(\mathrm{~s}, 1 \mathrm{H})$, $2.68(\mathrm{~s}, 1 \mathrm{H}), 1.63(\mathrm{~s}, 3 \mathrm{H}) ;{ }^{13} \mathrm{C} \mathrm{NMR}\left(\mathrm{CDCl}_{3}\right) \delta 175.4,135.9,129.0,128.0,127.8,77.1,69.8$, 24.4; IR (KBr) v 3482, 3427, 3224, $2105\left(-\mathrm{N}_{3}\right), 1664,1632 \mathrm{~cm}^{-1}(\mathrm{C}=\mathrm{O})$; MS (FAB) $\mathrm{m} / z .221$ $(\mathrm{M}+1)$; Anal. Calcd for $\mathrm{C}_{10} \mathrm{H}_{12} \mathrm{~N}_{4} \mathrm{O}_{2}$ : C, 54.54; H, 5.49 N, 25.44. Found: C, 54.59; H, 5.51; N, 25.52 . 


\section{Hydrogenation of $2 R, 3 R$-(-)-3-azido-2-hydroxy-2-methyl-3-phenylpropionamide 12 :}

Under hydrogen atmosphere, a mixture of 2R,3R-(-)-3-azido-2-hydroxy-2-methyl-3-phenylpropionamide $(1 \mathrm{mmol})$ and $\mathrm{Pd} / \mathrm{C}(10 \%$, $106.4 \mathrm{mg})$ in methanol $(20 \mathrm{ml})$ was stirred at room temperature for $2 \mathrm{~h}$. After filtration and concentration, the residue was recrystallized in methanol to give 2R,3R-(+)-3-amino-2-hydroxy-2-methyl-3-phenylpropionamide 13 as colorless crystals: yield 100\%; mp 156-157.5 $\square ;[\alpha]^{25}+44^{\circ}$ (c 1.0, DMSO); ee >99.5\% (chiral HPLC analysis); ${ }^{1} \mathrm{H}$ NMR (300MHz, DMSO-d $d_{6}$ TMS) $\delta 7.14-7.30(\mathrm{~m}, 5 \mathrm{H}), 3.91(\mathrm{~s}, 1 \mathrm{H}), 1.33(\mathrm{~s}, 3 \mathrm{H}) ;{ }^{13} \mathrm{C} \mathrm{NMR}$ $\left(\mathrm{DMSO}-d_{6}\right) \delta 177.3,143.0,128.2,127.1,126.3,76.9,60.5,23.7$; IR $(\mathrm{KBr}) \vee 3442$, 2984-3358, $1632 \mathrm{~cm}^{-1}(\mathrm{C}=\mathrm{O})$; MS (ESI) $\mathrm{m} / z 195(\mathrm{M}+1)$. Anal. Calcd for $\mathrm{C}_{10} \mathrm{H}_{14} \mathrm{~N}_{2} \mathrm{O}_{2}$ : C, 61.84; H, 7.27 N, 14.42. Found: C, 61.67; H, 7.16; N, 14.46.

Hydrolysis of $2 R, 3 R-(+)-3$-amino-2-hydroxy-2-methyl-3-phenylpropionamide 13 : $2 R, 3 R$-(+)-3-amino-2-hydroxy-2-methyl-3-phenylpropionamide $(0.7 \mathrm{mmol})$ was refluxed in hydrochloric acid $(6 \mathrm{~N}, 20 \mathrm{ml})$ for $10 \mathrm{~h}$. The reaction mixture was diluted with a small amount of water and extracted with ethyl acetate. The aqueous phase was concentrated and the residue was purified using a ion exchange resin column to afford pure 2R,3R-(+)-3-amino-2-hydroxy-2-methyl-3-phenylpropionic acid 14 as white solid: yield 92\%; mp 198-200 $\square ;[\alpha]^{25}{ }_{\mathrm{D}}+24^{\circ}\left(c\right.$ 1.0, $\left.\mathrm{H}_{2} \mathrm{O}\right)$; ee $>99.5 \%$ (chiral HPLC analysis); ${ }^{1} \mathrm{H}$ NMR (300MHz, DMSO- $\left.d_{6}, \mathrm{TMS}\right) \delta 8.91(\mathrm{~s}, \mathrm{br}, 1 \mathrm{H}), 7.32-7.44(\mathrm{~m} \square 5 \mathrm{H}), 4.13(\mathrm{~s}, 1 \mathrm{H}), 1.10(\mathrm{~s}, 3 \mathrm{H})$; ${ }^{13} \mathrm{C}$ NMR (DMSO- $\left.d_{6}\right) \delta 176.2,135.4,128.6,127.8,127.6,72.2,59.9,21.6$; IR (KBr) v 3448, 2932-3056, $1592 \mathrm{~cm}^{-1}$; MS (FAB) $\mathrm{m} / z .196(\mathrm{M}+1)$. Anal. Calcd for $\mathrm{C}_{10} \mathrm{H}_{13} \mathrm{NO}_{3}$ : C, 61.53; $\mathrm{H}$, 6.71; N, 7.18. Found: C, 61.28; H, 6.75; N, 6.87.

Synthesis of 2S,3S-(+)-2-amino-3-hydroxy-2-methyl-3-phenylpropionic acid (17). 
A mixture of 2R,3R-(-)-3-azido-2-hydroxy-2-methyl-3-phenylpropionamide 12 (2 mmol) and $\mathrm{Ph}_{3} \mathrm{P}(2.1 \mathrm{mmol})$ in $\mathrm{DMF}(40 \mathrm{ml})$ was stirred at room temperature until no evolution of nitrogen was observed. The mixture was then heated at $100{ }^{\circ} \mathrm{C}$ for $6 \mathrm{~h}$. After removal of solvent under vacuum, the residue was subjected to a silica gel column using a mixture of petroleum ether and ethyl acetate $(1: 1)$ as an eluent. 2S,3R-(+)-2-Methyl-3-phenylaziridine-2-carboxamide 15 was obtained as white solid: yield $65 \% ;[\alpha]^{25}+121^{\circ}\left(c 2.0, \mathrm{CH}_{3} \mathrm{OH}\right) ;{ }^{1} \mathrm{H}$ NMR $\left(300 \mathrm{MHz}, \mathrm{DMSO}-d_{6}\right) \delta 7.58(\mathrm{~s}, 1 \mathrm{H}), 7.45(\mathrm{~s}$, 1H), 7.23-7.35 (m, 5H), $3.13(\mathrm{~d}, 1 \mathrm{H}, J=9.6 \mathrm{~Hz}), 2.26(\mathrm{~d}, 1 \mathrm{H}, J=9.6 \mathrm{~Hz}), 1.02(\mathrm{~s}, 3 \mathrm{H}) ;{ }^{13} \mathrm{C}$ NMR (DMSO- $\left.d_{6}\right) \delta 174.1,136.9,127.9,127.7,126.9,43.1,40.9,13.6$; IR $(\mathrm{KBr}) \vee 3496$, 3328, 3292, 3257, 1673, $1662 \mathrm{~cm}^{-1}$; MS (ESI) $\mathrm{m} / \mathrm{z} .177(\mathrm{M}+1)$; Anal. Calcd for $\mathrm{C}_{10} \mathrm{H}_{12} \mathrm{~N}_{2} \mathrm{O}: \mathrm{C}$, 68.18; H, 6.86; N, 15.90. Found: C, 67.79; H, 6.85; N, 15.83. Aziridine 15 (1 mmol) was then heated with $\mathrm{TsOH}(1.1 \mathrm{mmol})$ at $100{ }^{\circ} \mathrm{C}$ for $8 \mathrm{~h}$ in a mixture of $\mathrm{THF}(7 \mathrm{ml})$ and water $(7 \mathrm{ml})$. After reaction, the solvent was removed and the solid residue was dissolved in water $(20 \mathrm{ml})$ and washed with ethyl acetate $(3 \times 8 \mathrm{ml})$. The aqueous solution was concentrated to dryness, and hydrochloric acid $(6 \mathrm{~N}, 20 \mathrm{ml})$ was added. After refluxing for $4 \mathrm{~h}$, the solvent was removed under vacuum. The residue was purified with an ion exchange column to give pure $2 S, 3 S$-(+)-2-amino-3-hydroxy-2-methyl-3-phenylpropionic acid 17: yield 87\%; $[\alpha]^{25}+13.9^{\circ}$ $\left(c 0.36, \mathrm{H}_{2} \mathrm{O}\right)\left[\right.$ lit. $\left.^{25}[\alpha]^{25}{ }_{\mathrm{D}}+12.6^{\circ}\left(c 0.36, \mathrm{H}_{2} \mathrm{O}\right)\right]$; ee $>99.5 \%$ (chiral HPLC analysis); ${ }^{1} \mathrm{H}$ NMR $\left(300 \mathrm{MHz}, \mathrm{H}_{2} \mathrm{O}\right) \delta$ 7.29-7.31 (m, 5H), $4.96(\mathrm{~s}, 1 \mathrm{H}), 1.52(\mathrm{~s}, 3 \mathrm{H}) ;{ }^{13} \mathrm{C} \mathrm{NMR}\left(\mathrm{H}_{2} \mathrm{O}\right) \delta 174.3$, 137.5, 128.8, 128.6, 126.7, 74.6, 65.9, 19.9; IR (KBr) v 3257, 2495-3110, 1636, $1592 \mathrm{~cm}^{-1}$. An overall yield of $79.5 \%$ was obtained for $\mathbf{1 7}$ when aziridine intermediate $\mathbf{1 5}$ was not isolated and purified. 
2. ${ }^{1} \mathrm{H}$ and ${ }^{13} \mathrm{C}$ NMR spectra of $2,1 \mathrm{a}, 4 \mathrm{a}, 4 \mathrm{e}, 5 \mathrm{a}, 6-15,17$. 

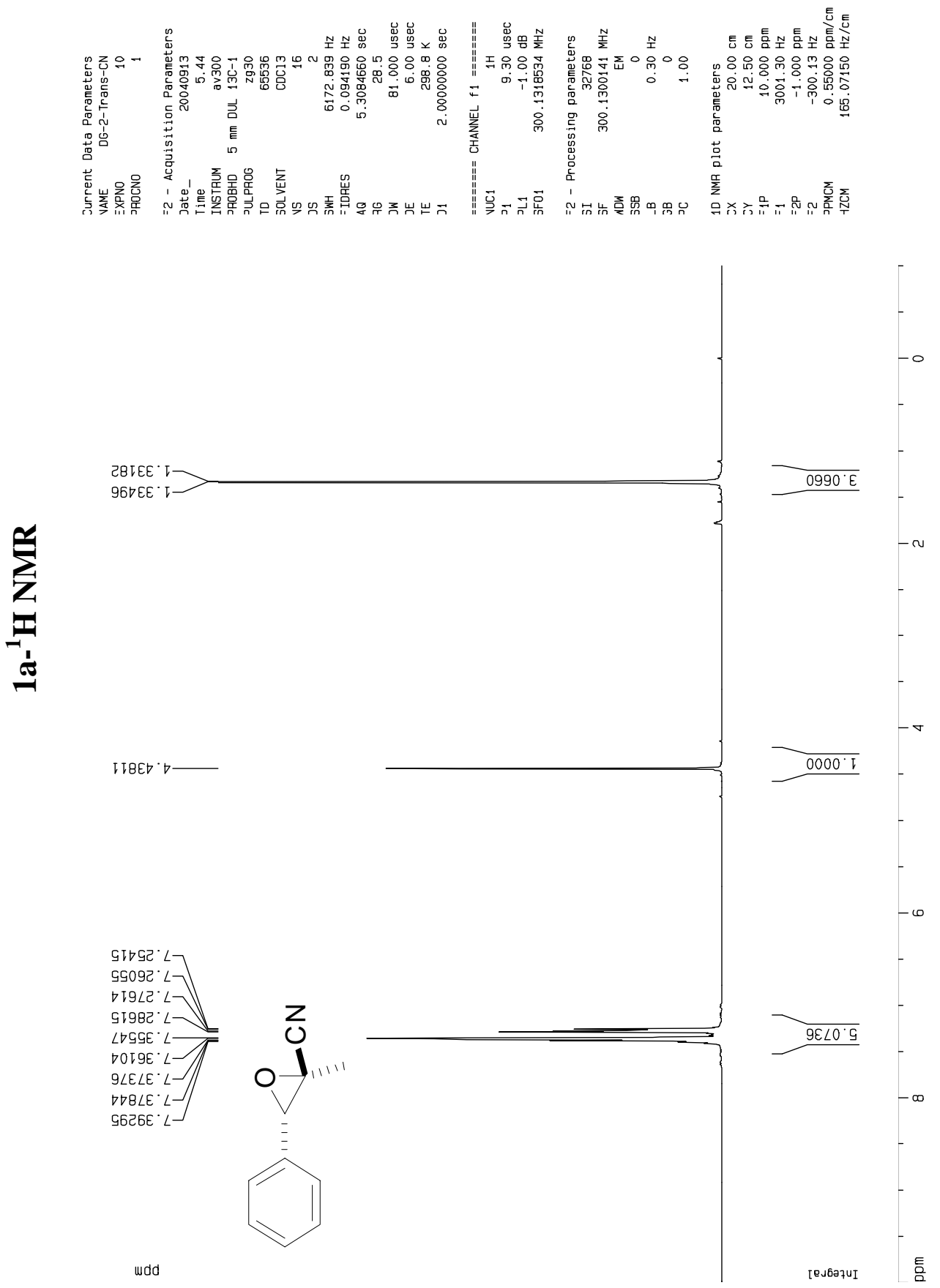


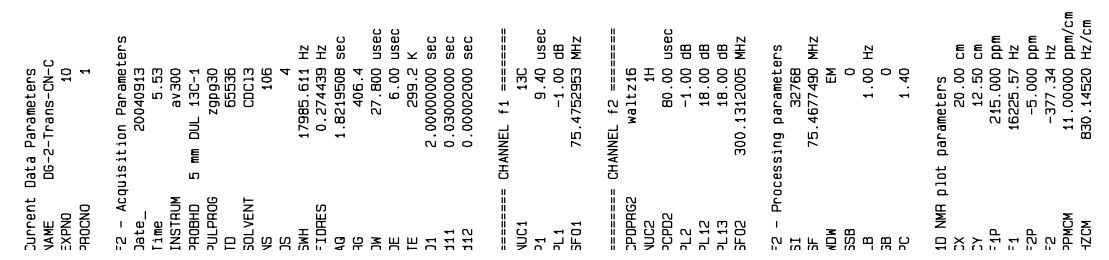

OGI'GI
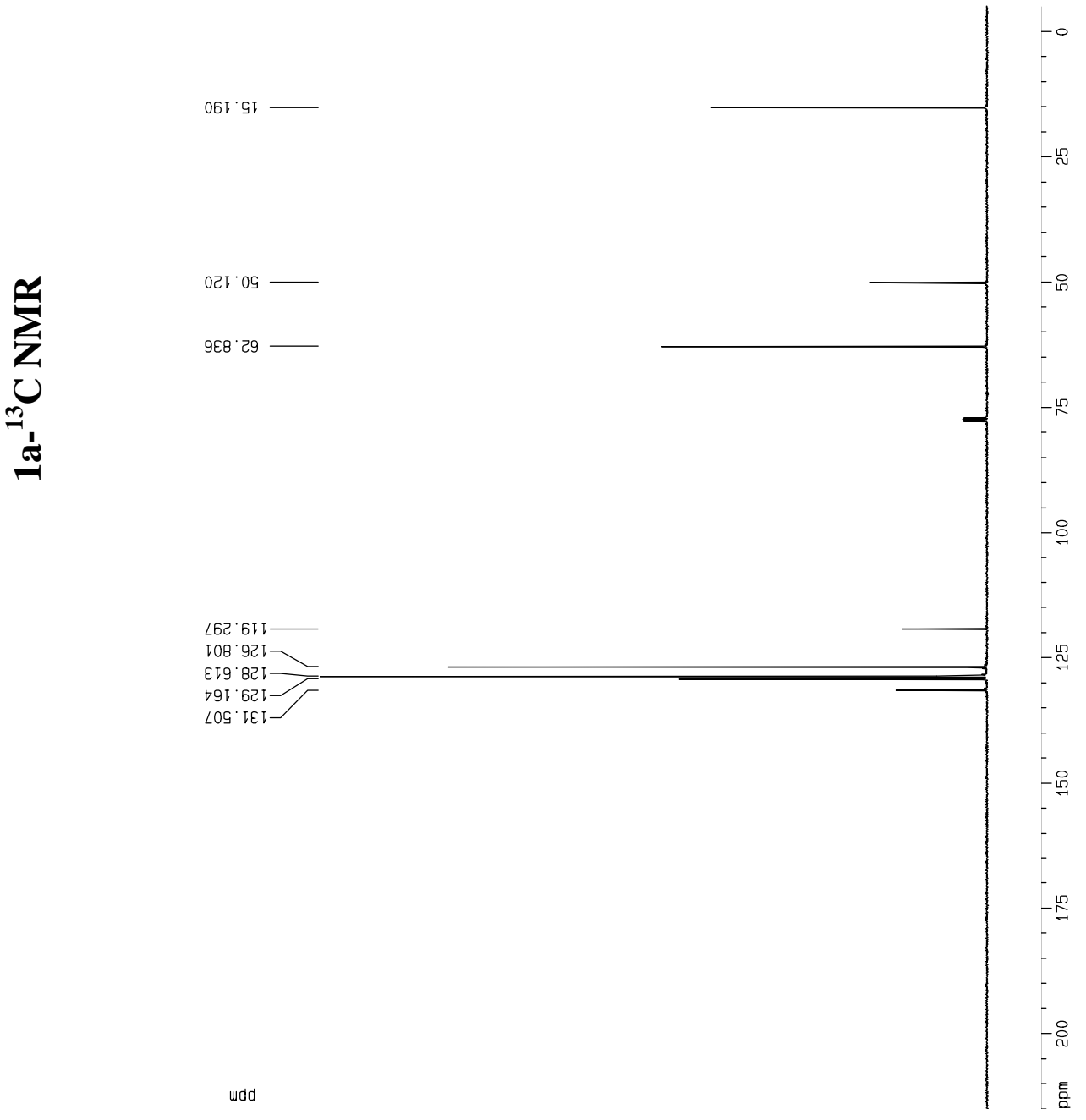

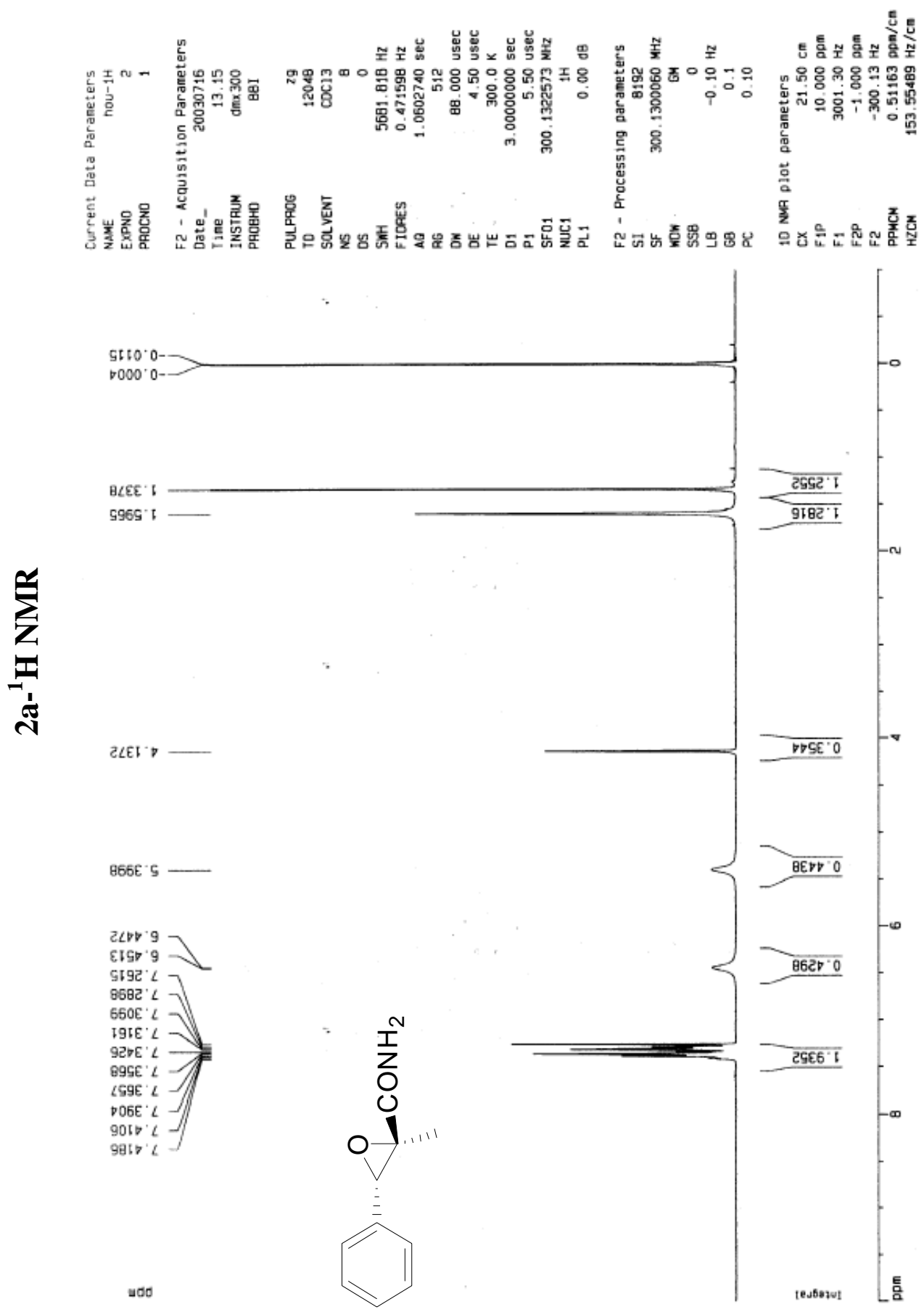


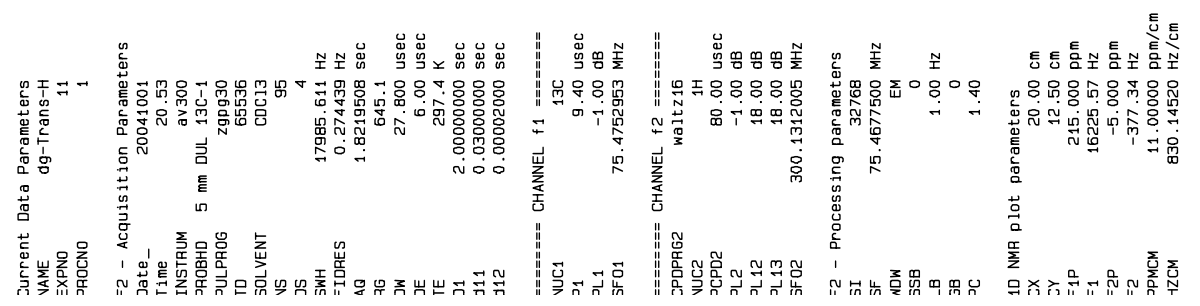

268' I

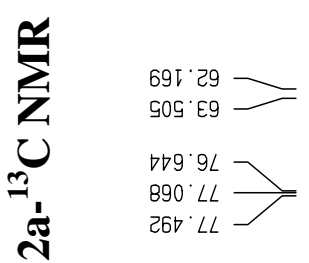

689.95 ז

ITE BटT

B8E $\mathrm{BCL} \longrightarrow$

BSt EEI-

GDI $\forall \angle L$

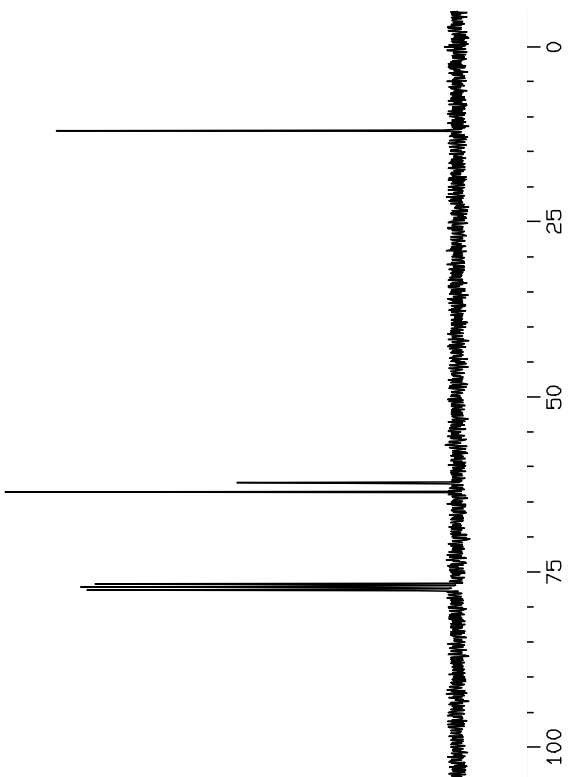

$-\stackrel{-1}{-1}$

$\stackrel{-9}{-1}$

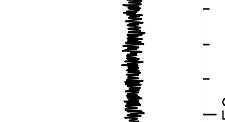



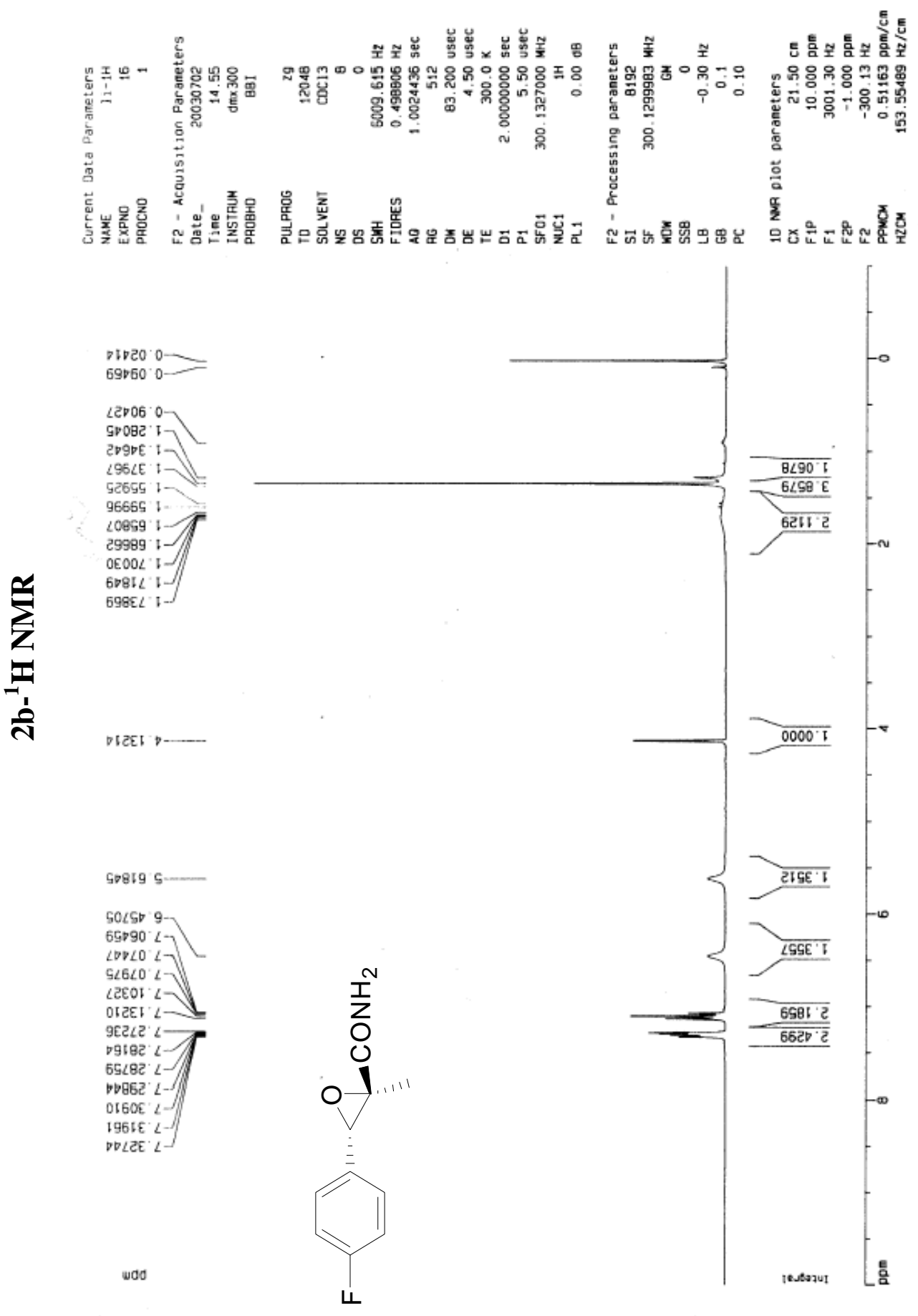


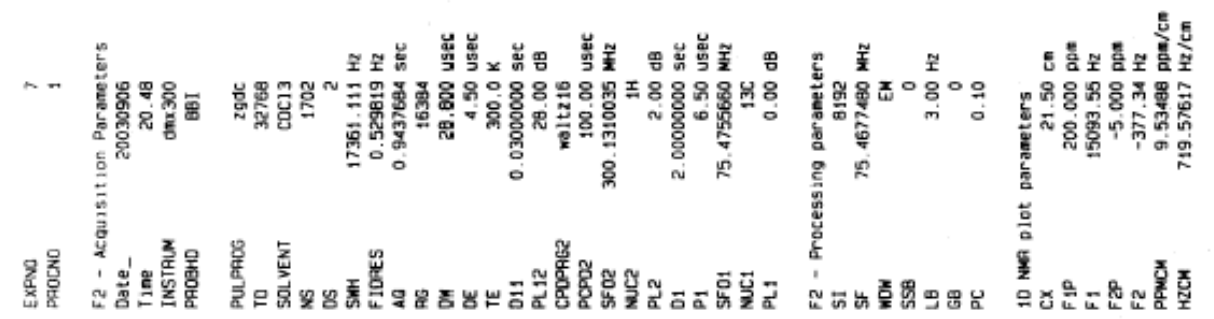

6. 11
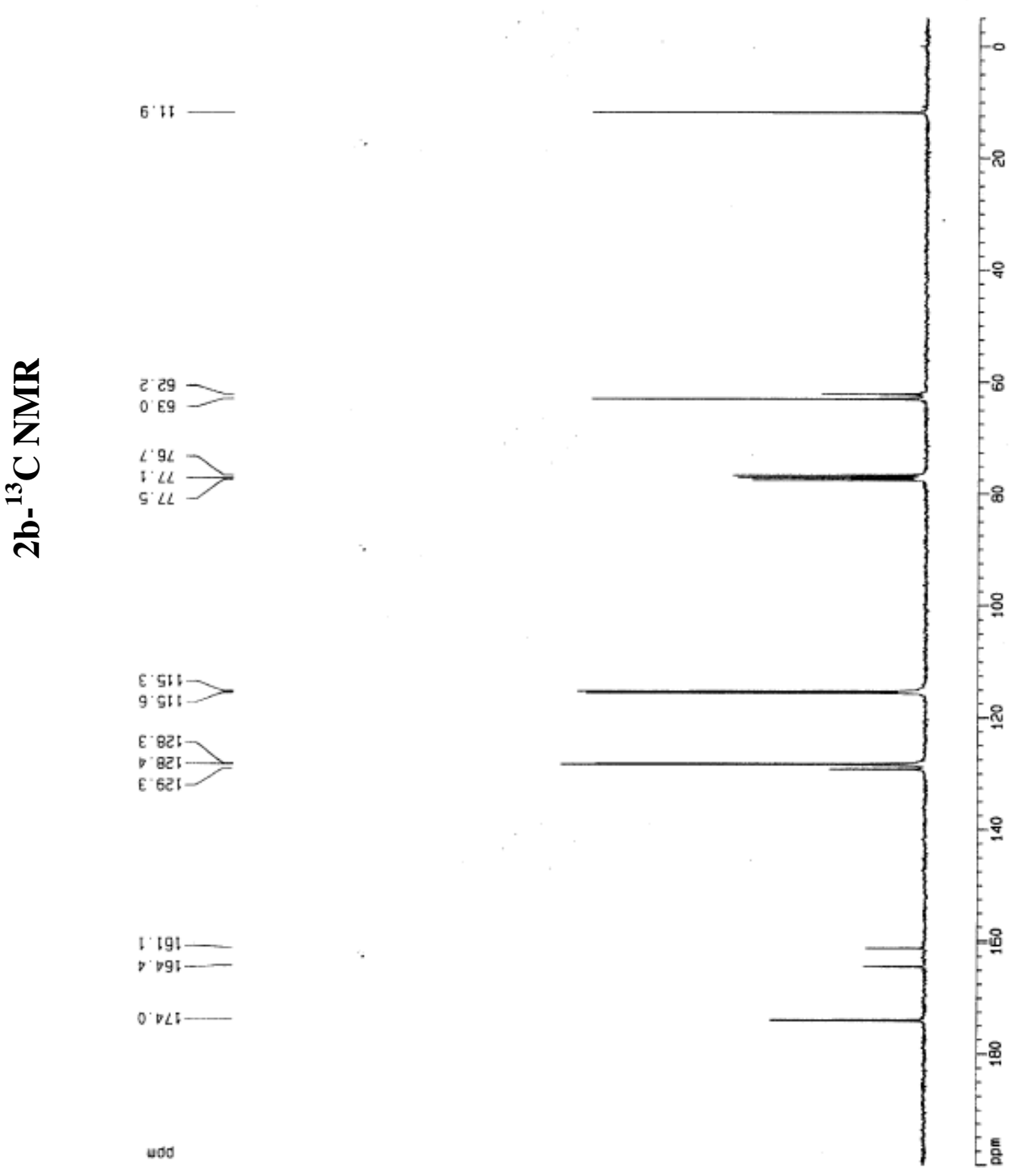

udo

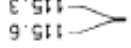

E. 825

$\therefore-851>$

E.621

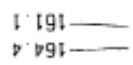

$00 \angle 5$ 

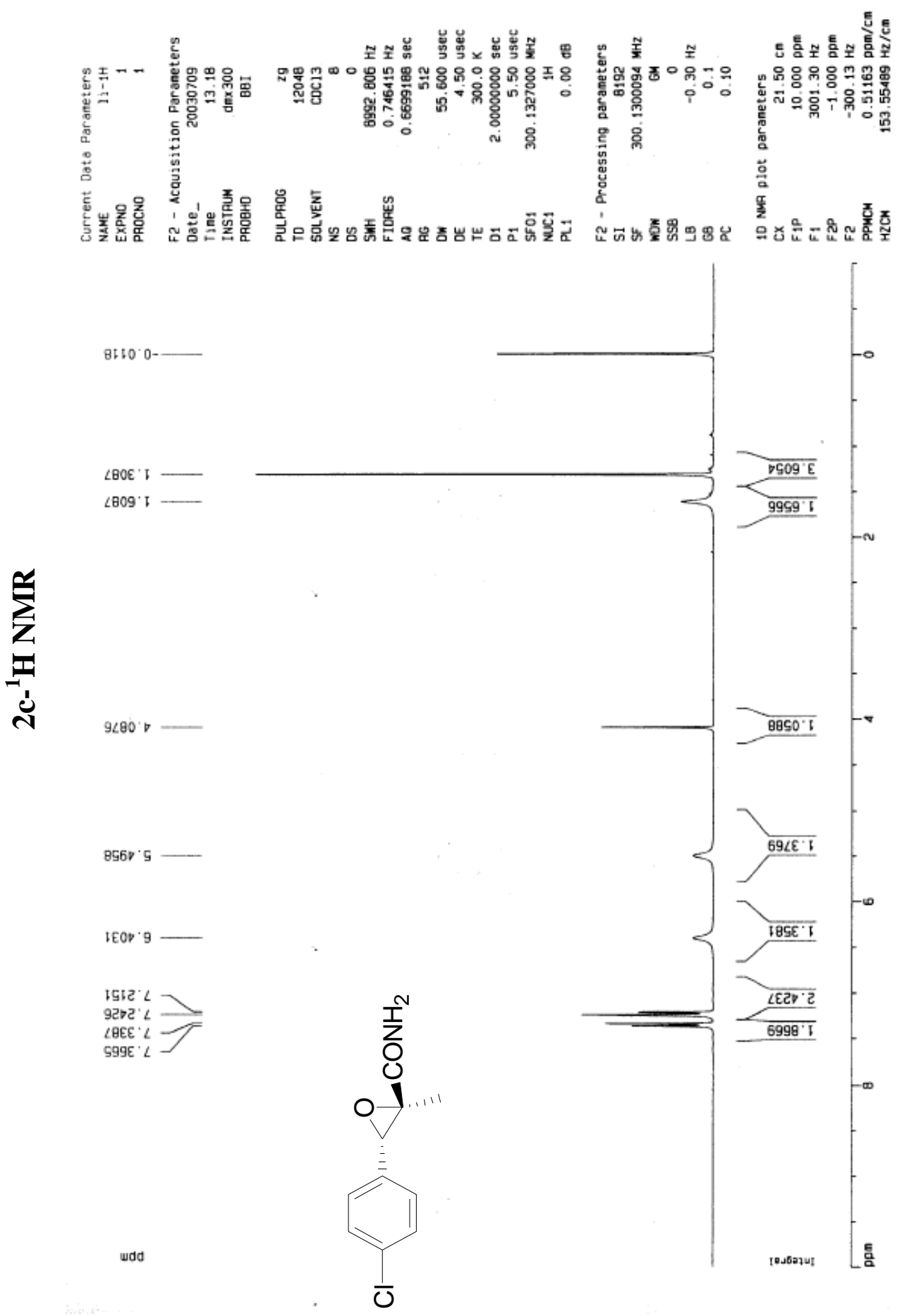


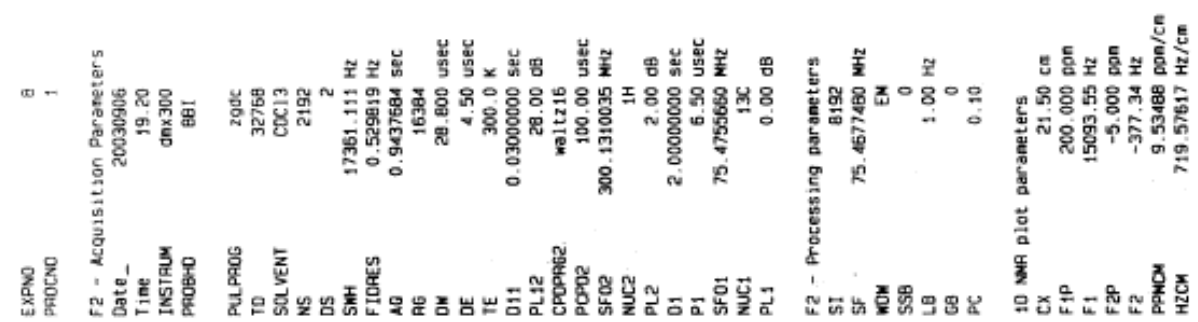

6. 11
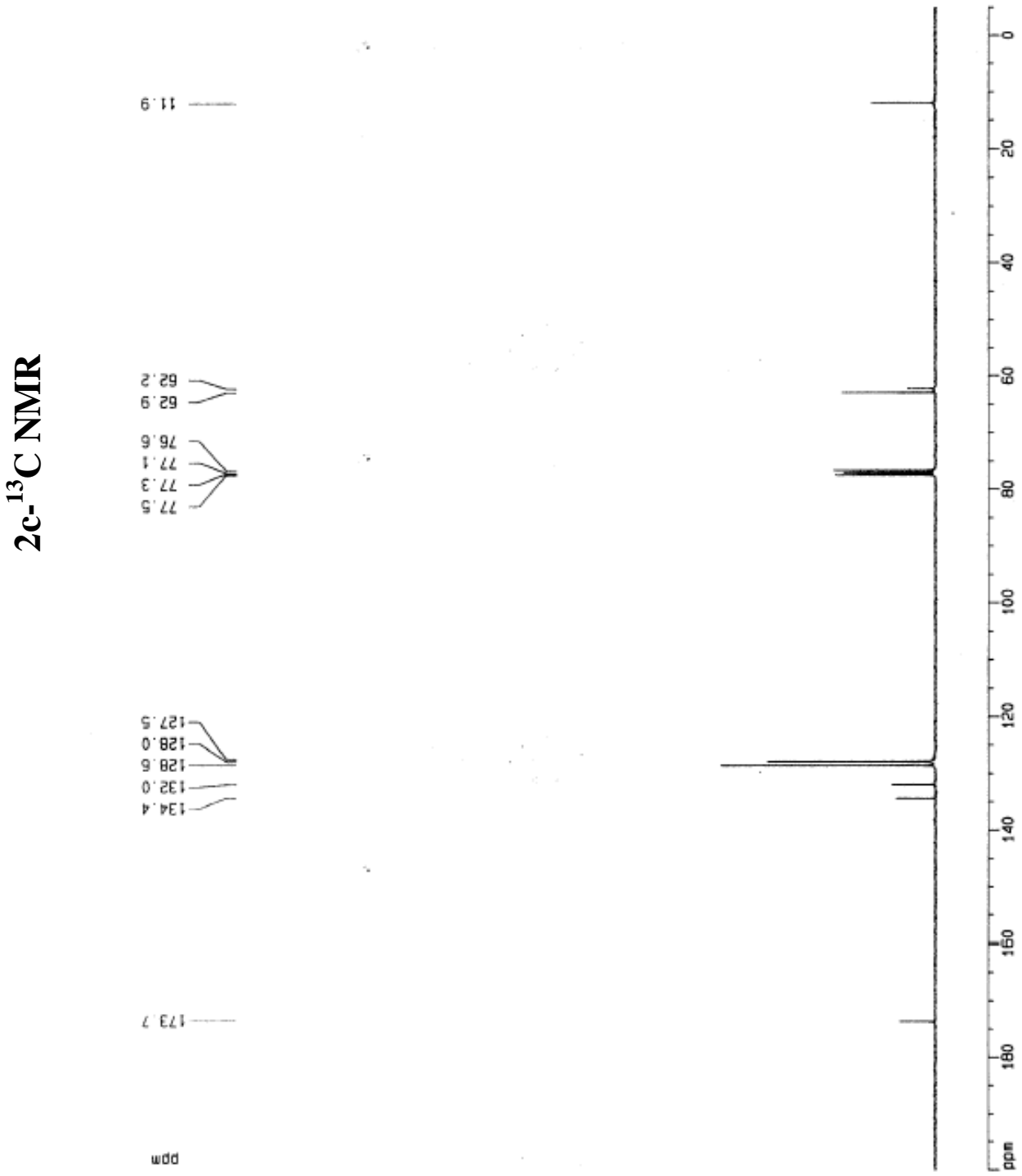

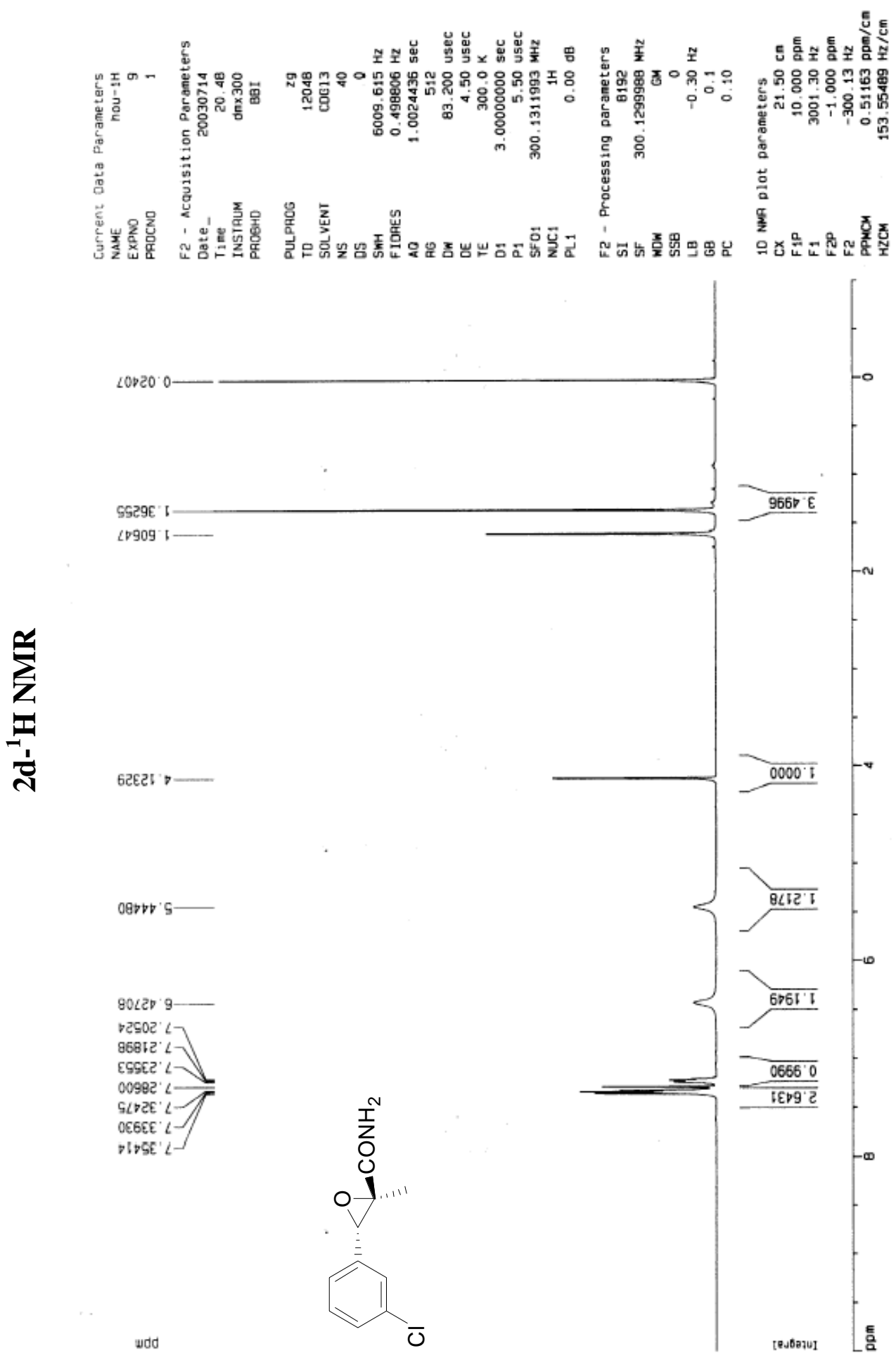

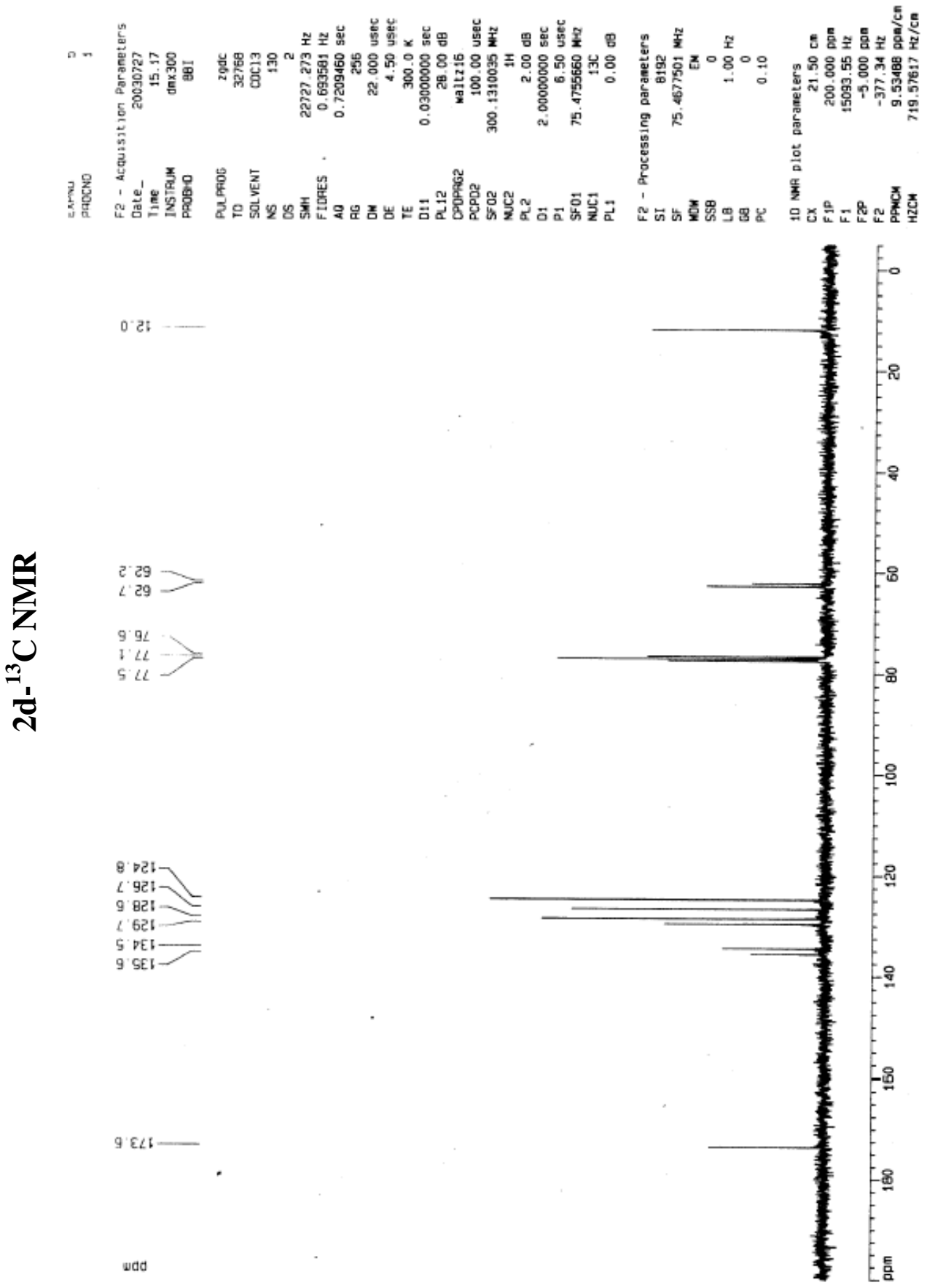

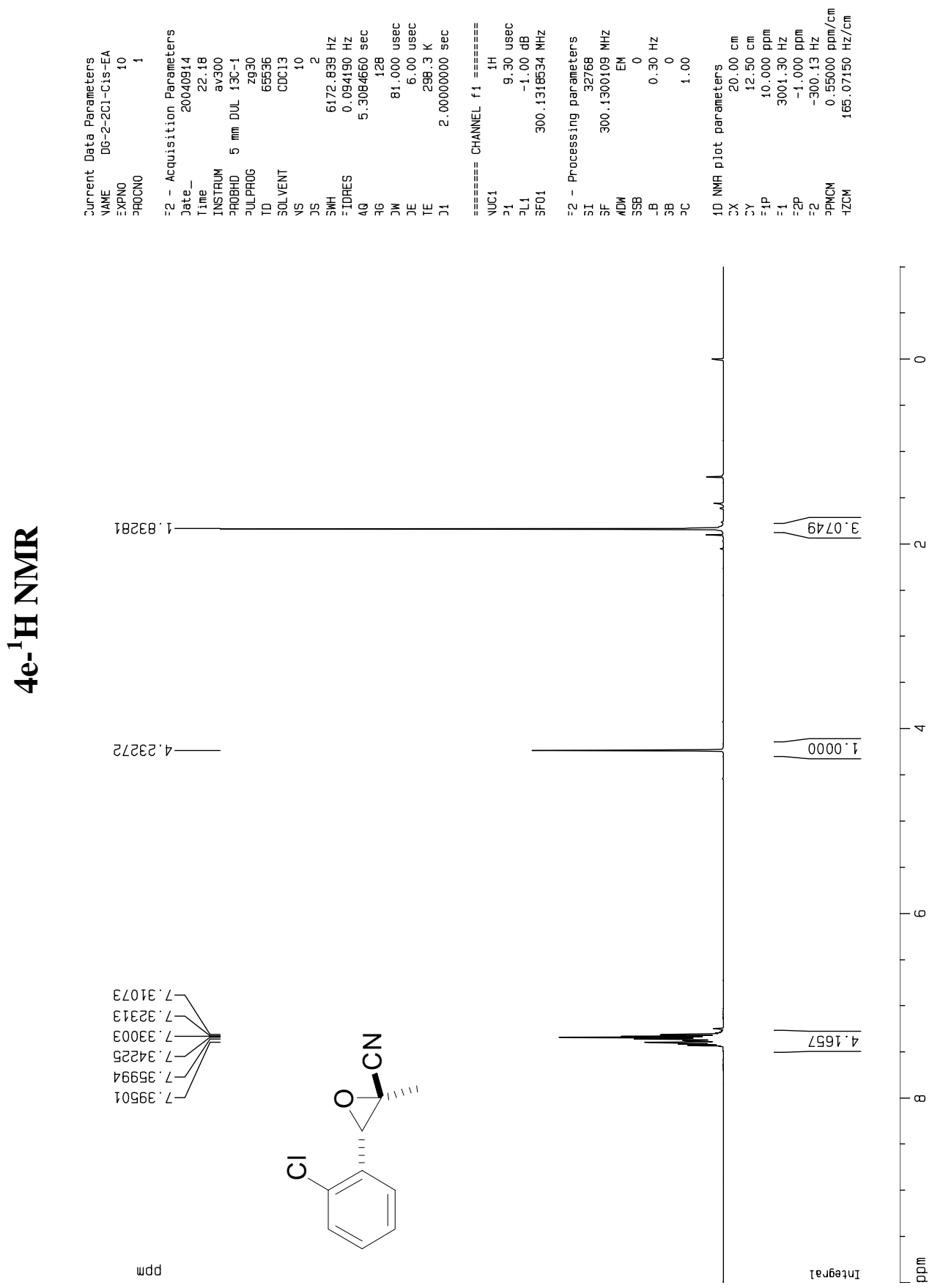


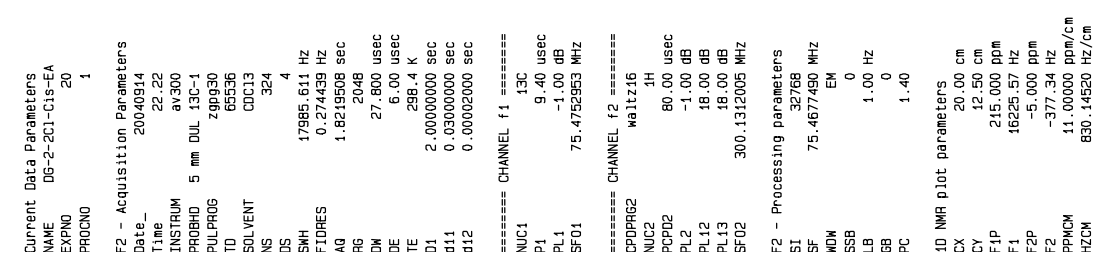

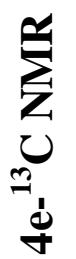
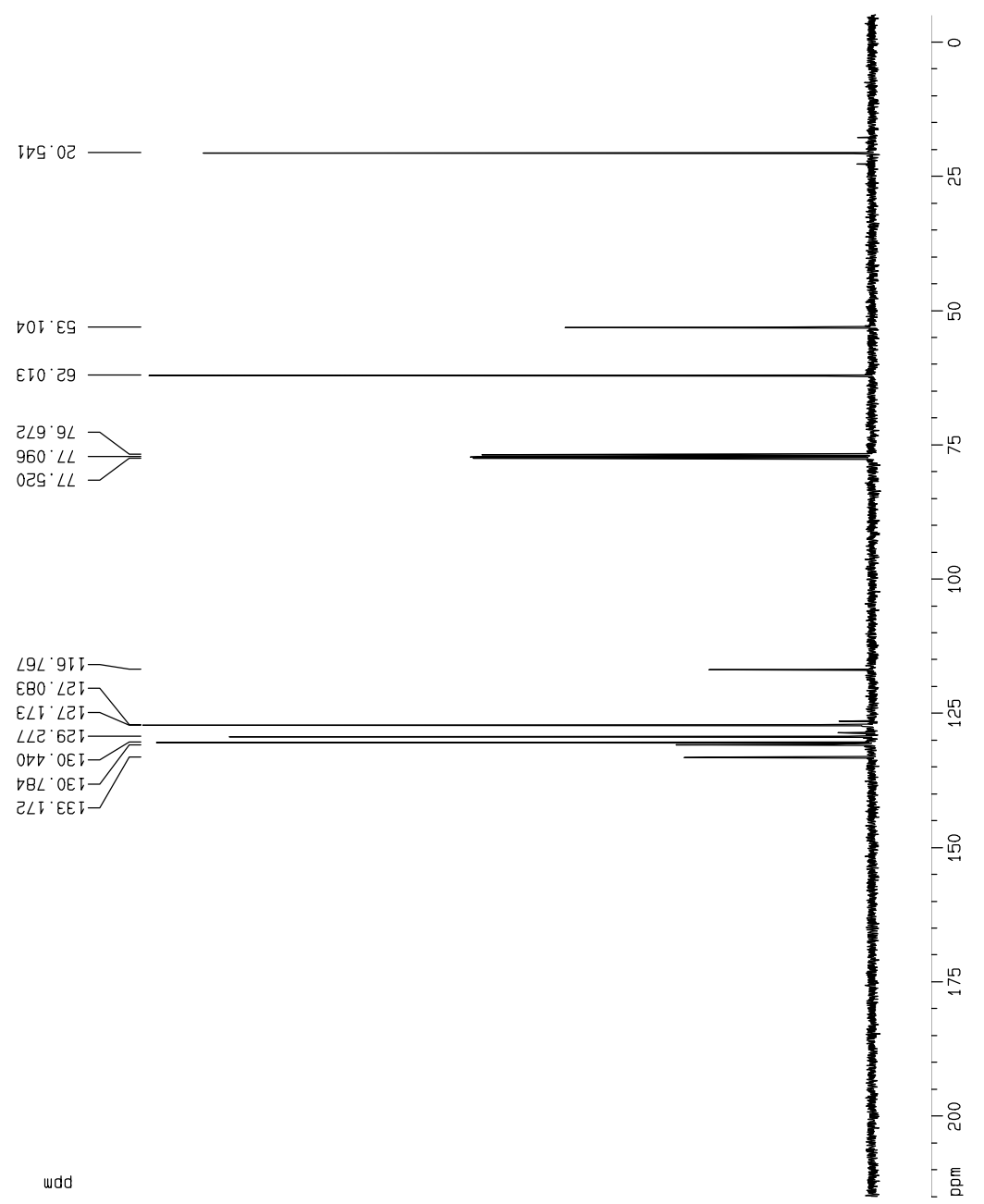

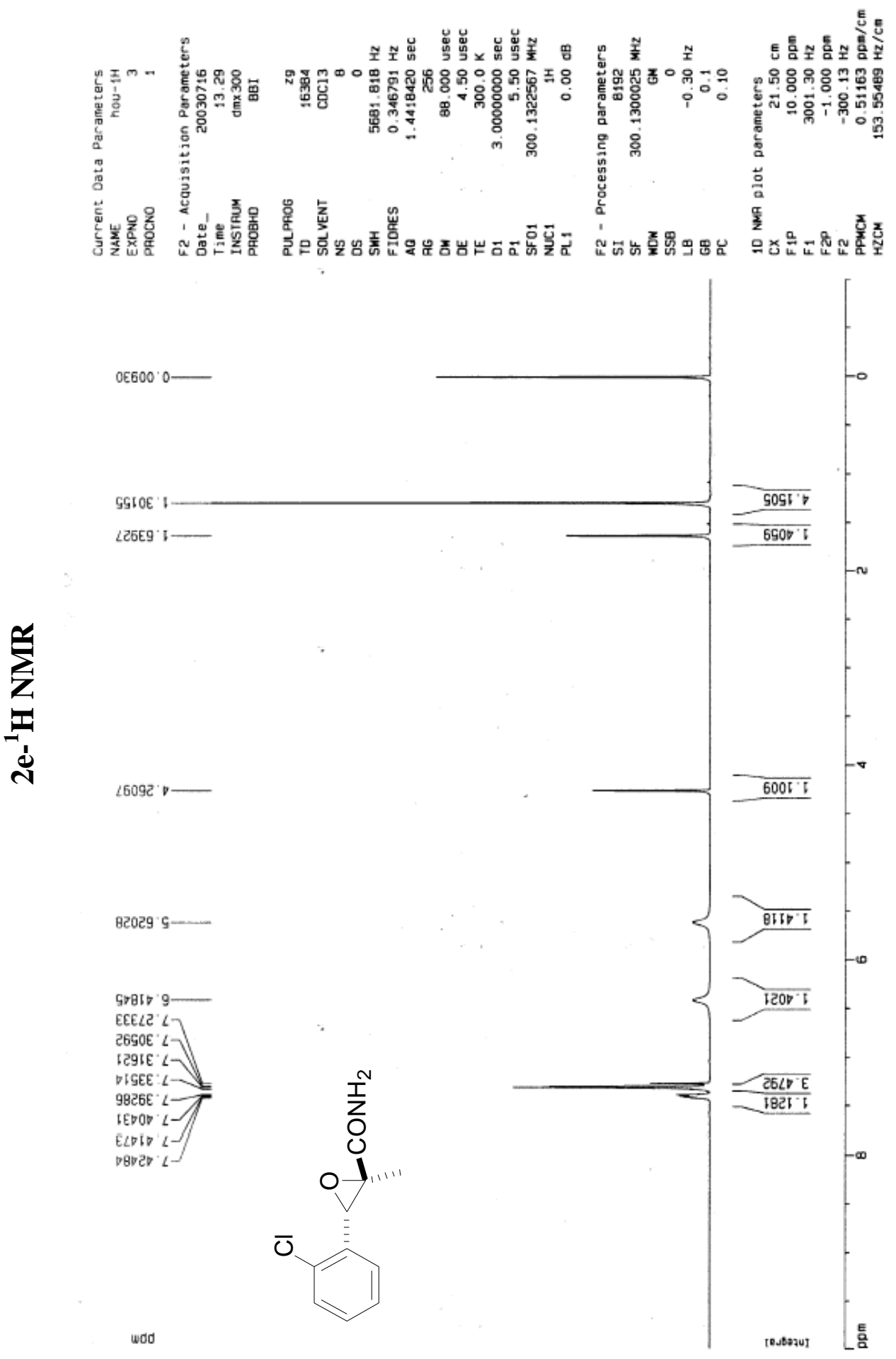

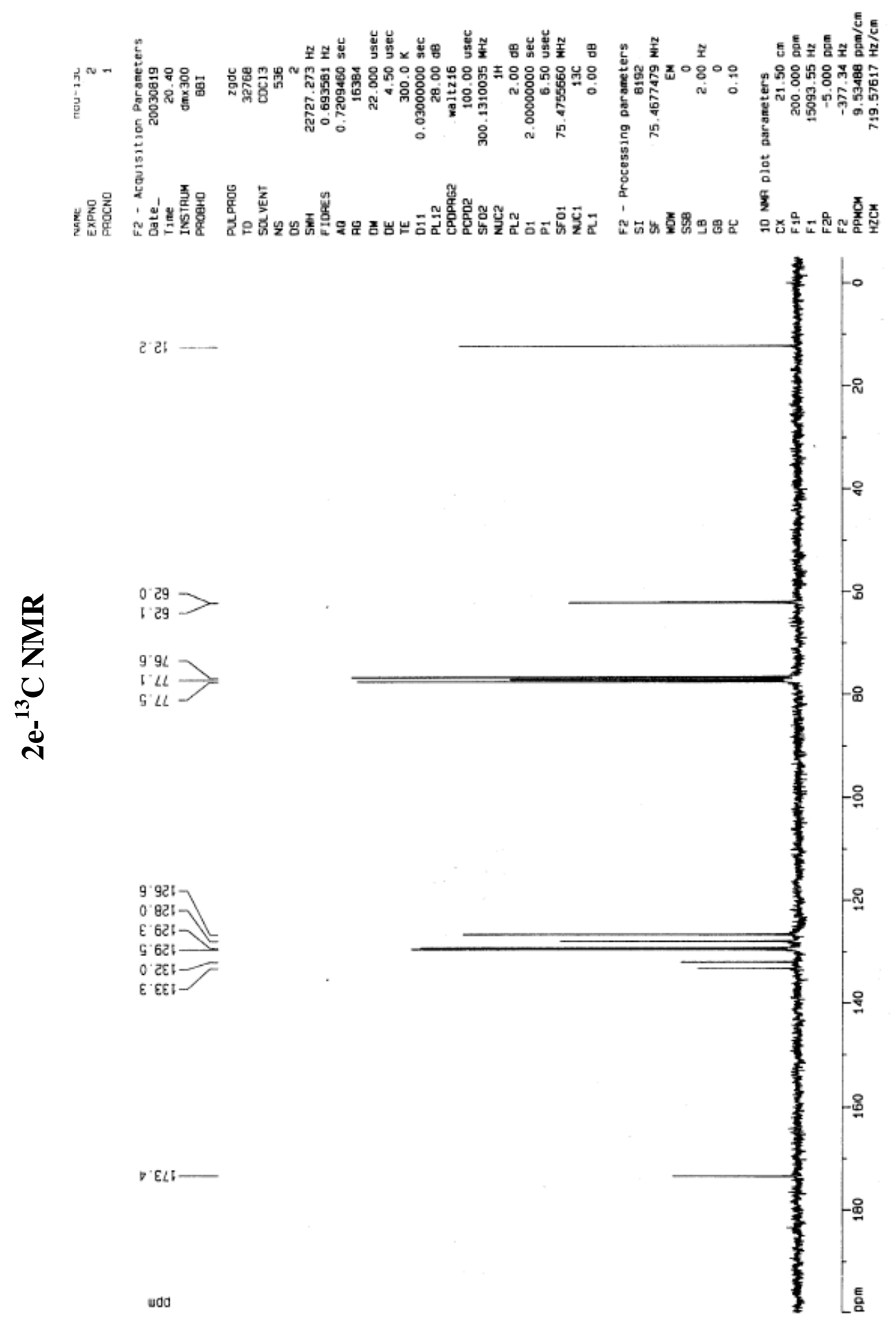

udd 

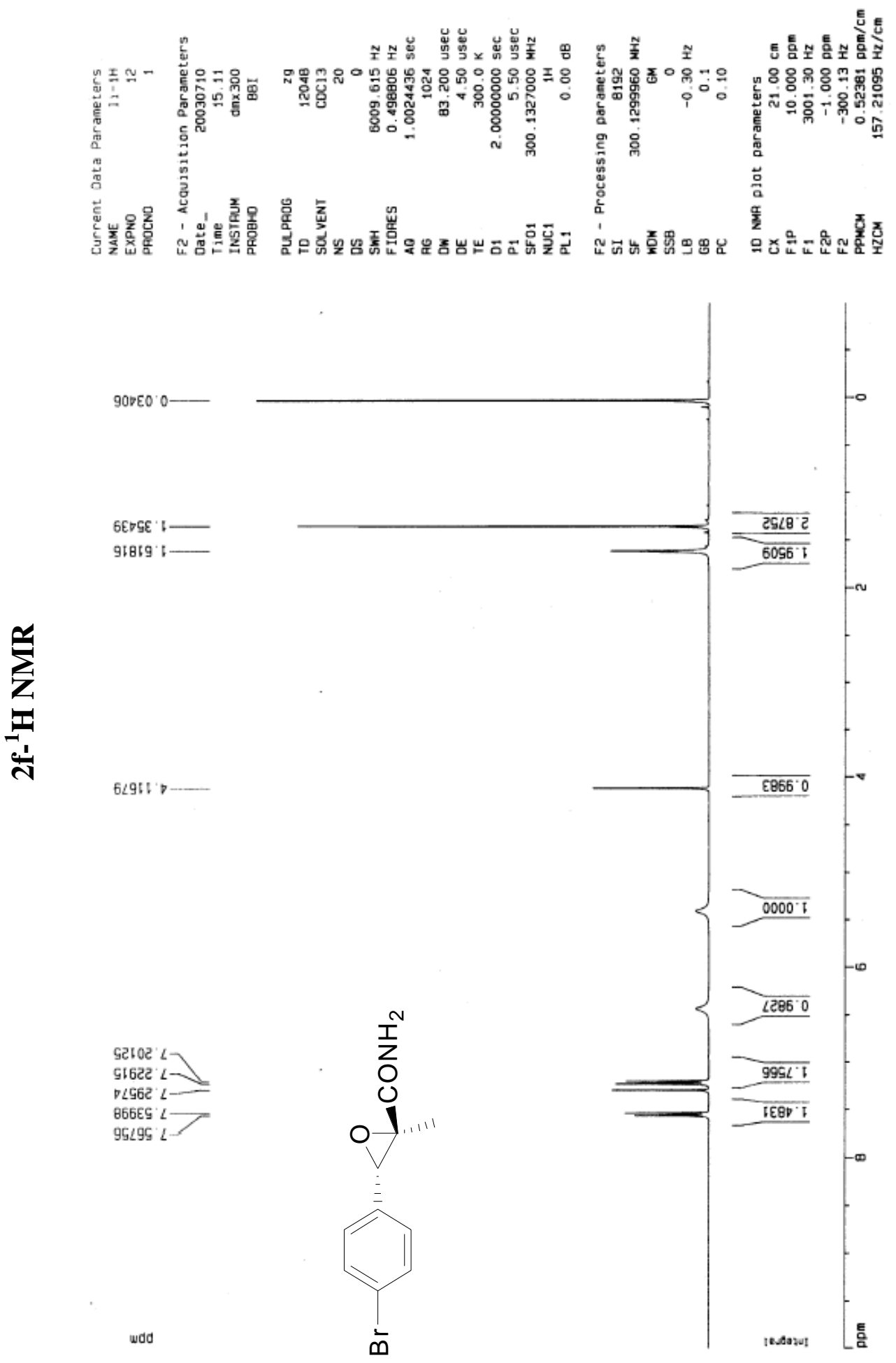

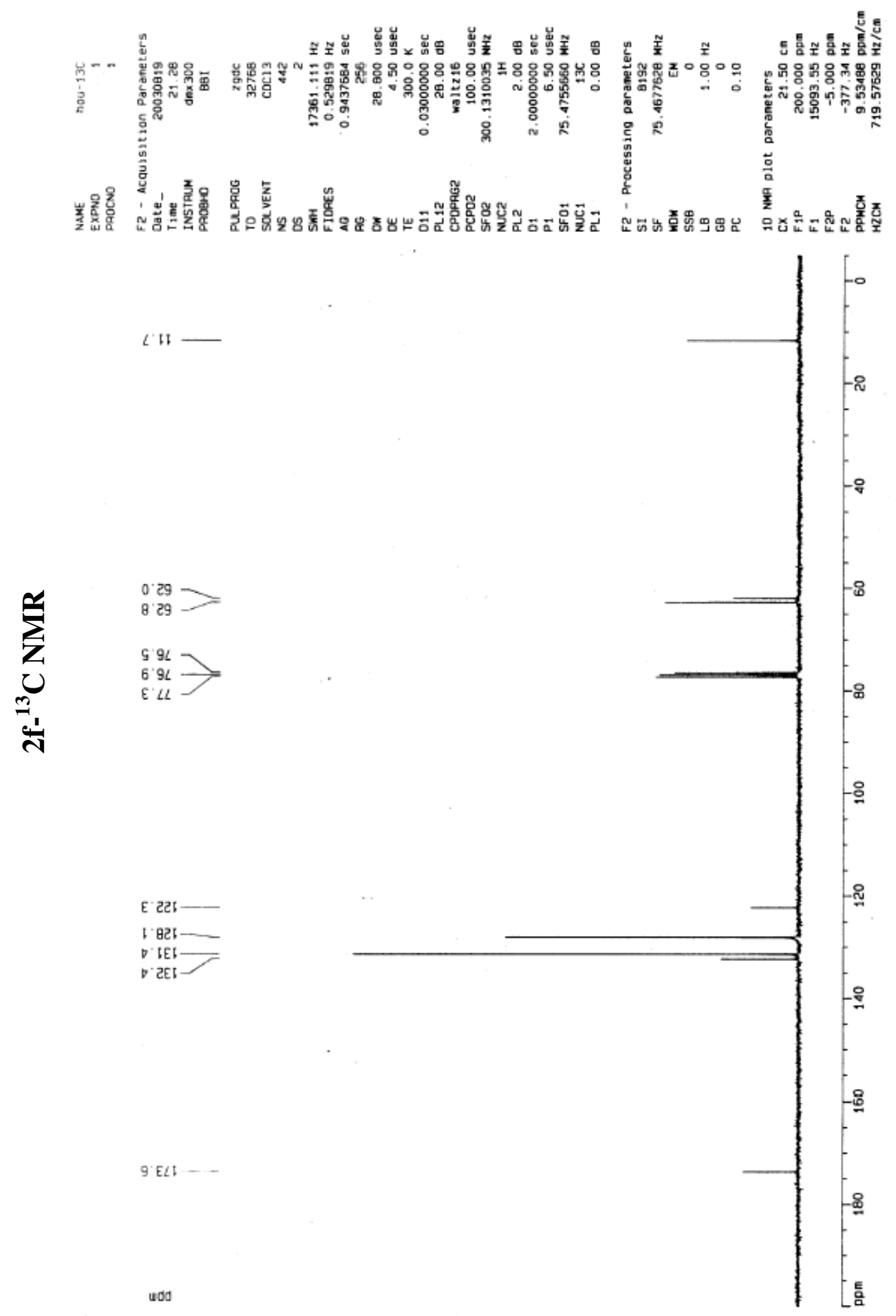

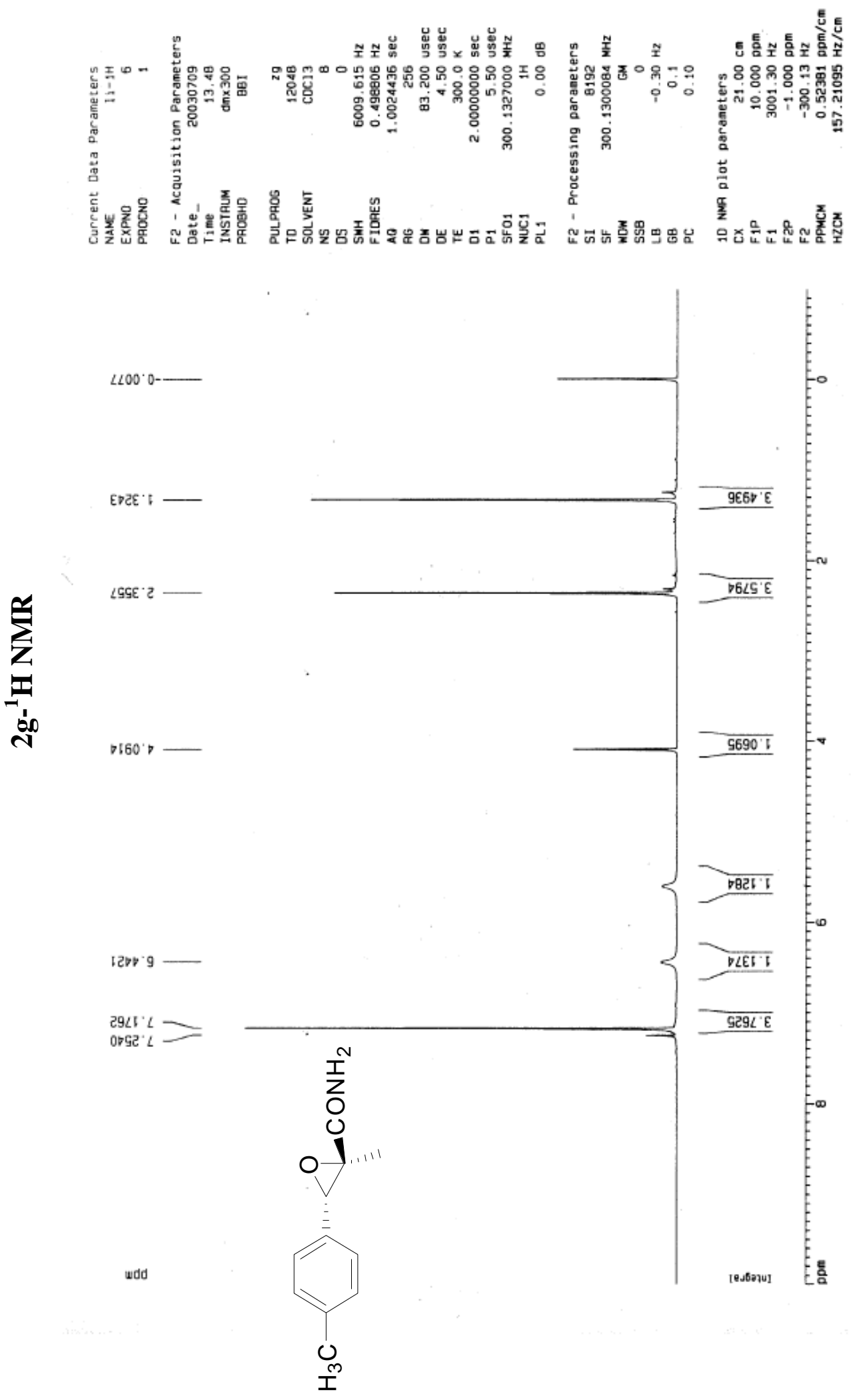

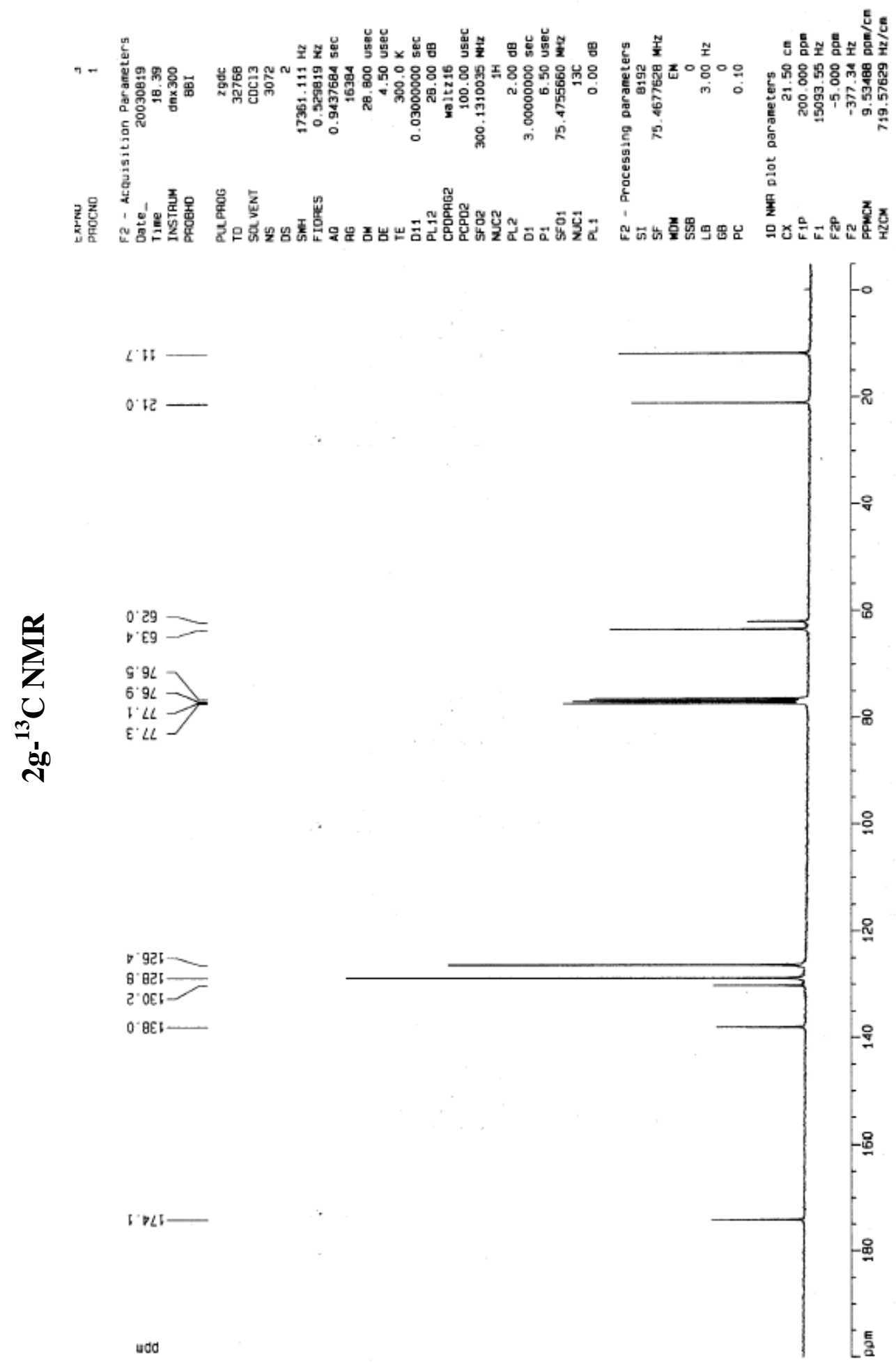

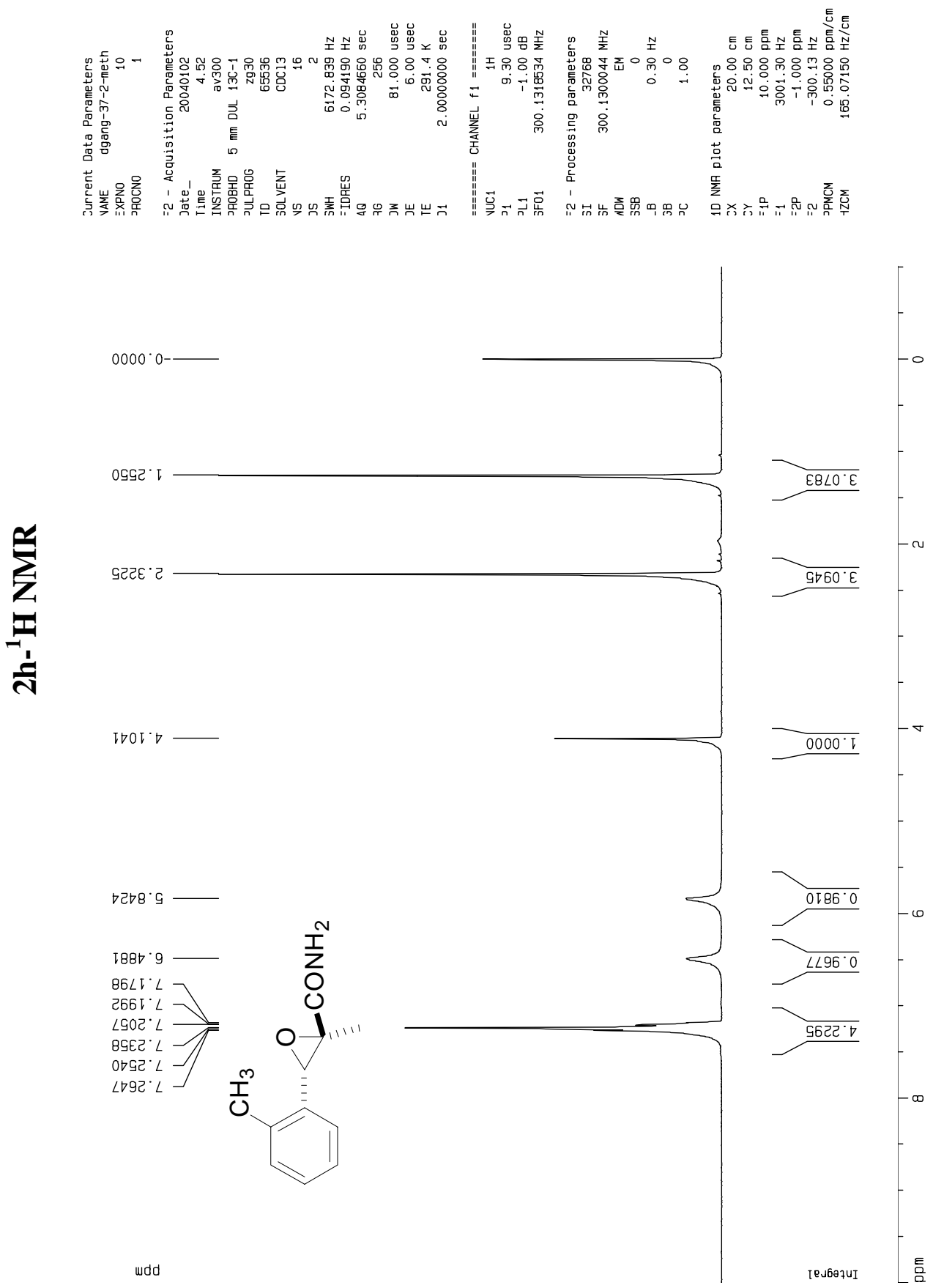


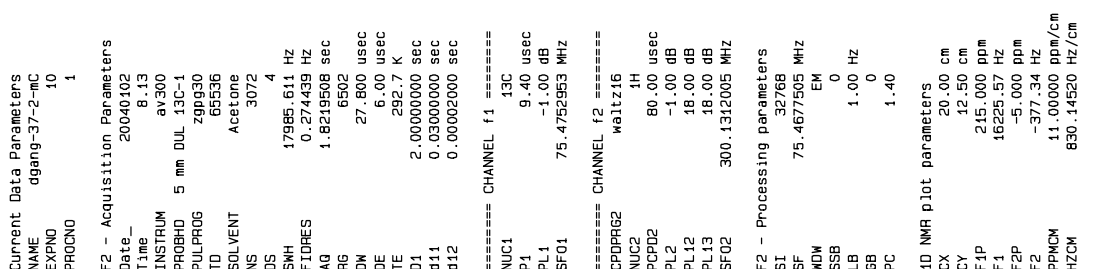

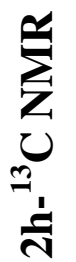
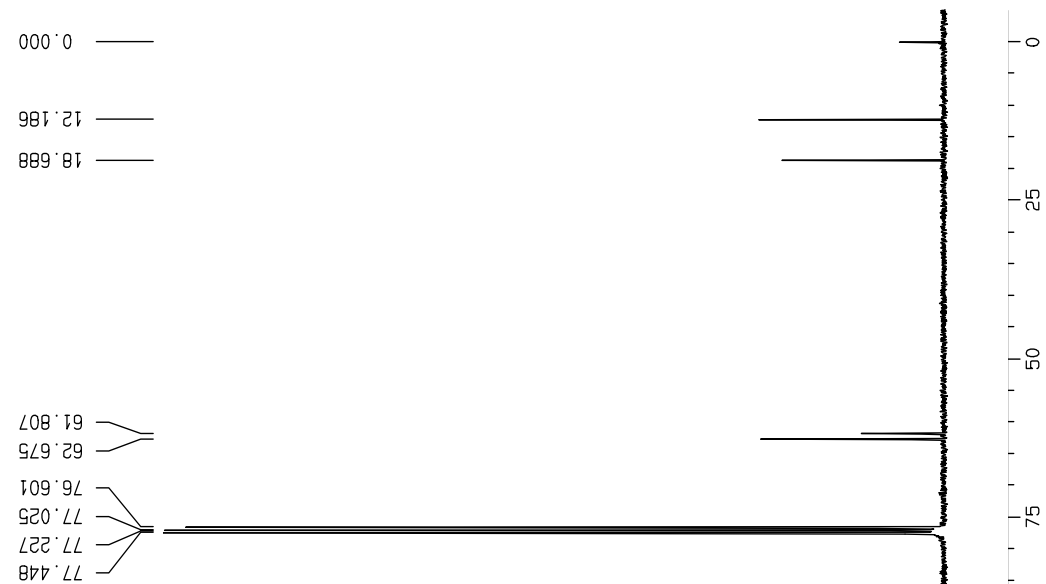

98I टा

889.81

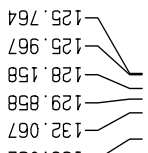

टEO $9 E I-$

$008^{\circ} E \angle I$ 

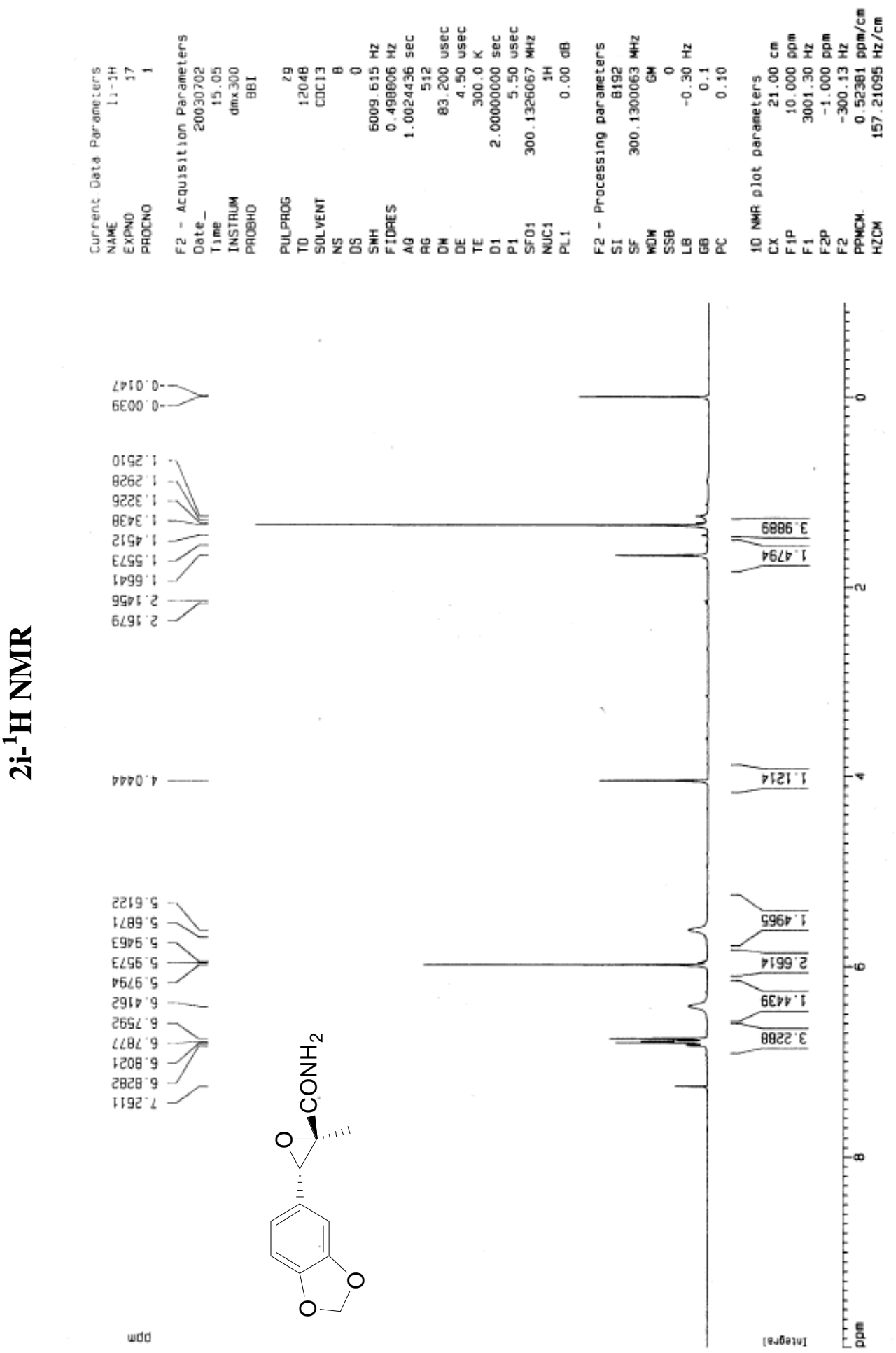


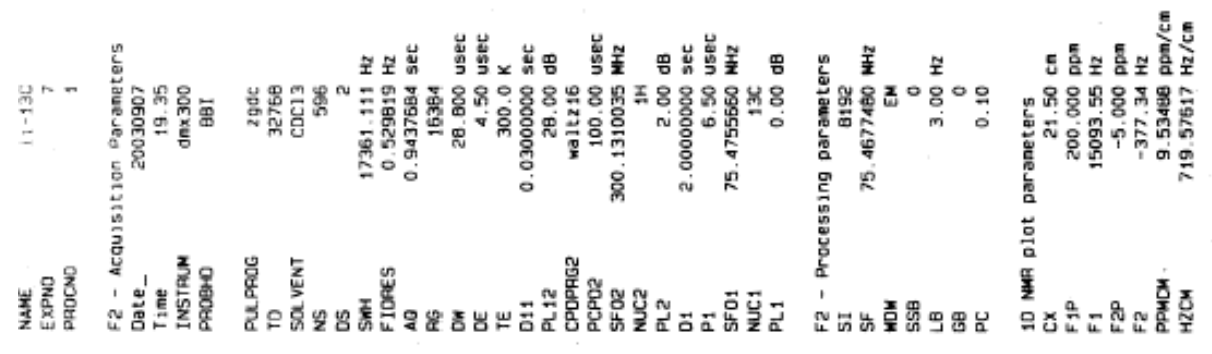

$8: 11$
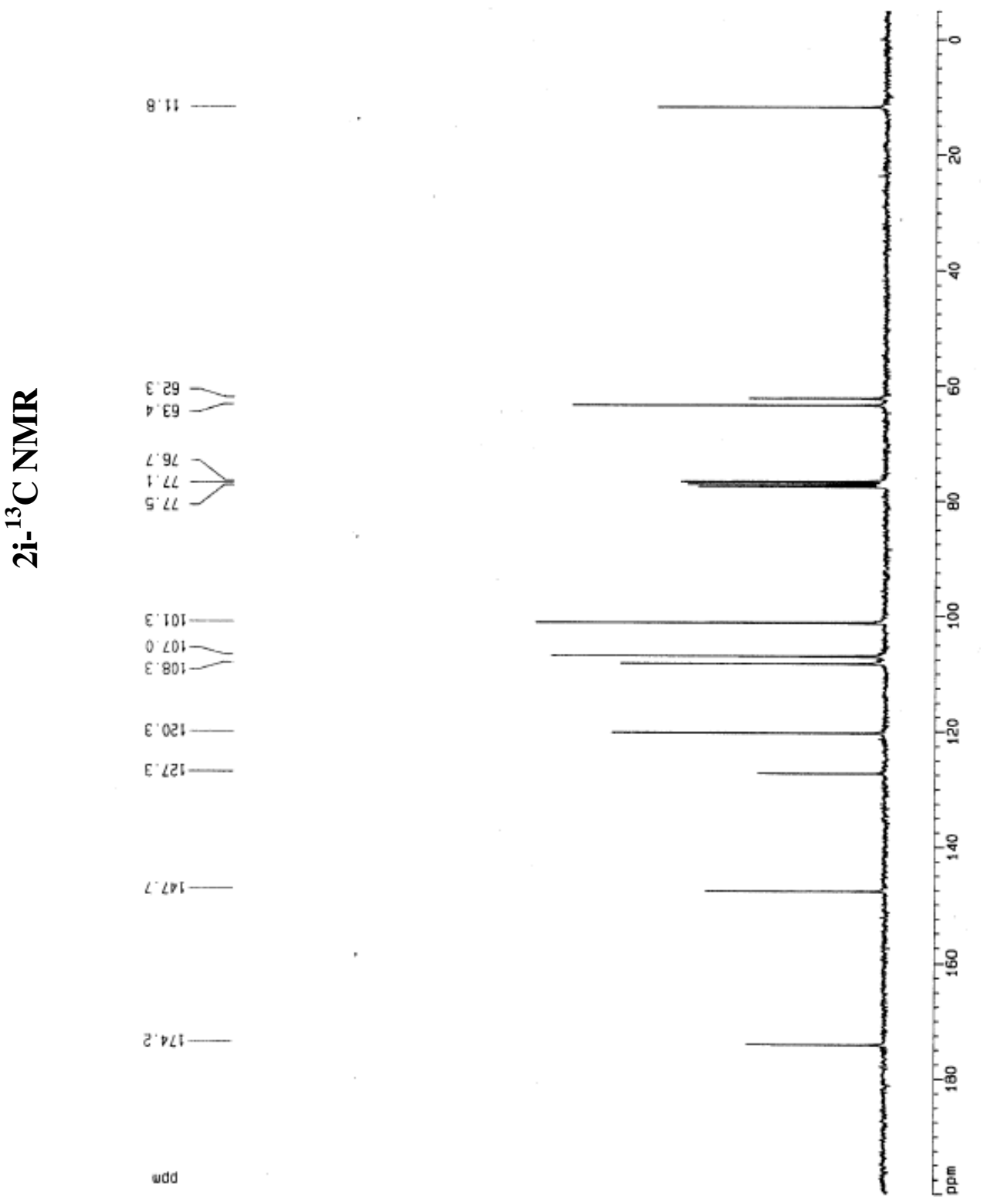

E' IDI-

$0 . \mathrm{OOH}=$

E'021-

E' $\angle 25-$

$\angle \angle O L-$

$2 \cdot D L 1-$ 

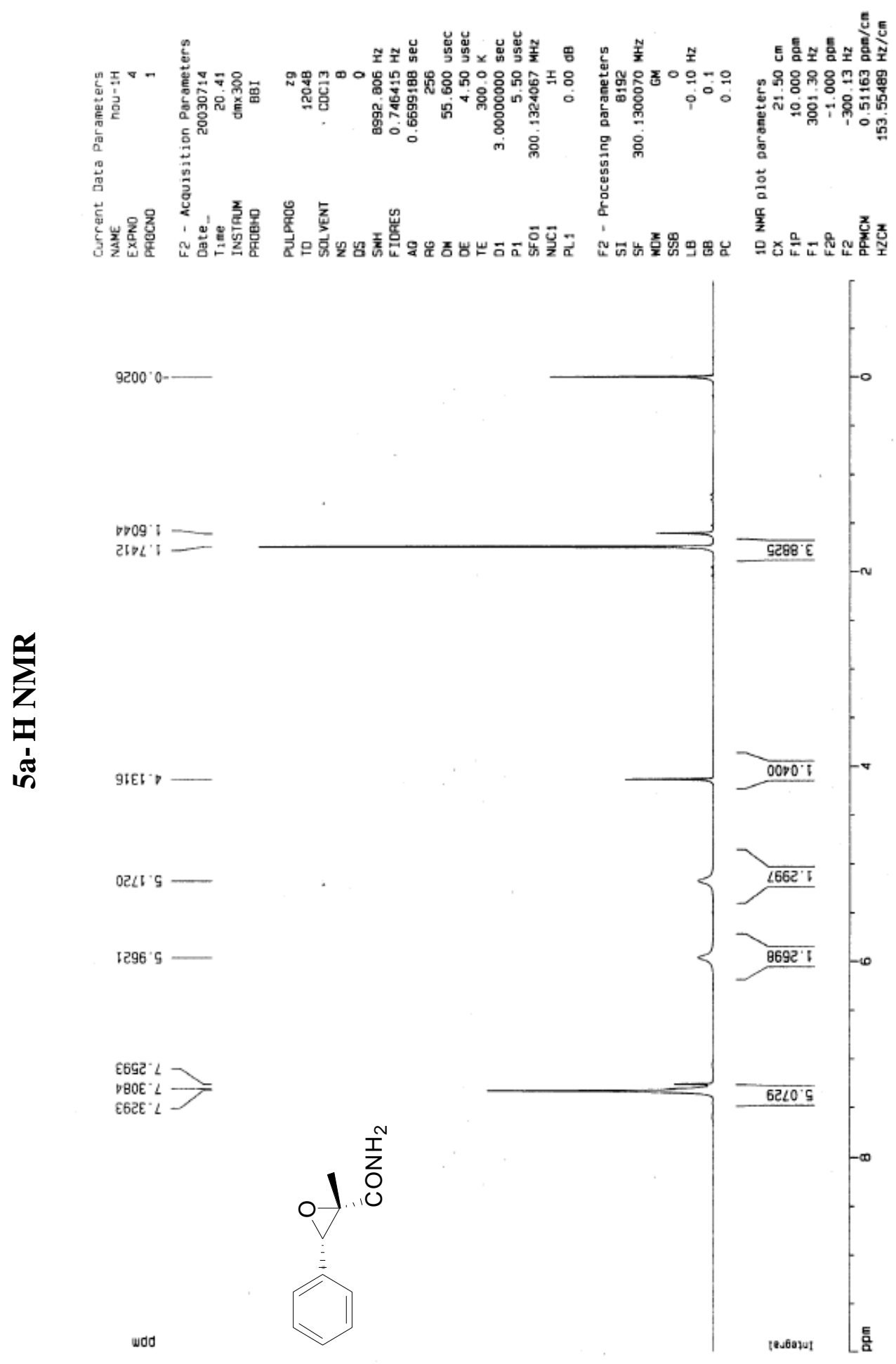

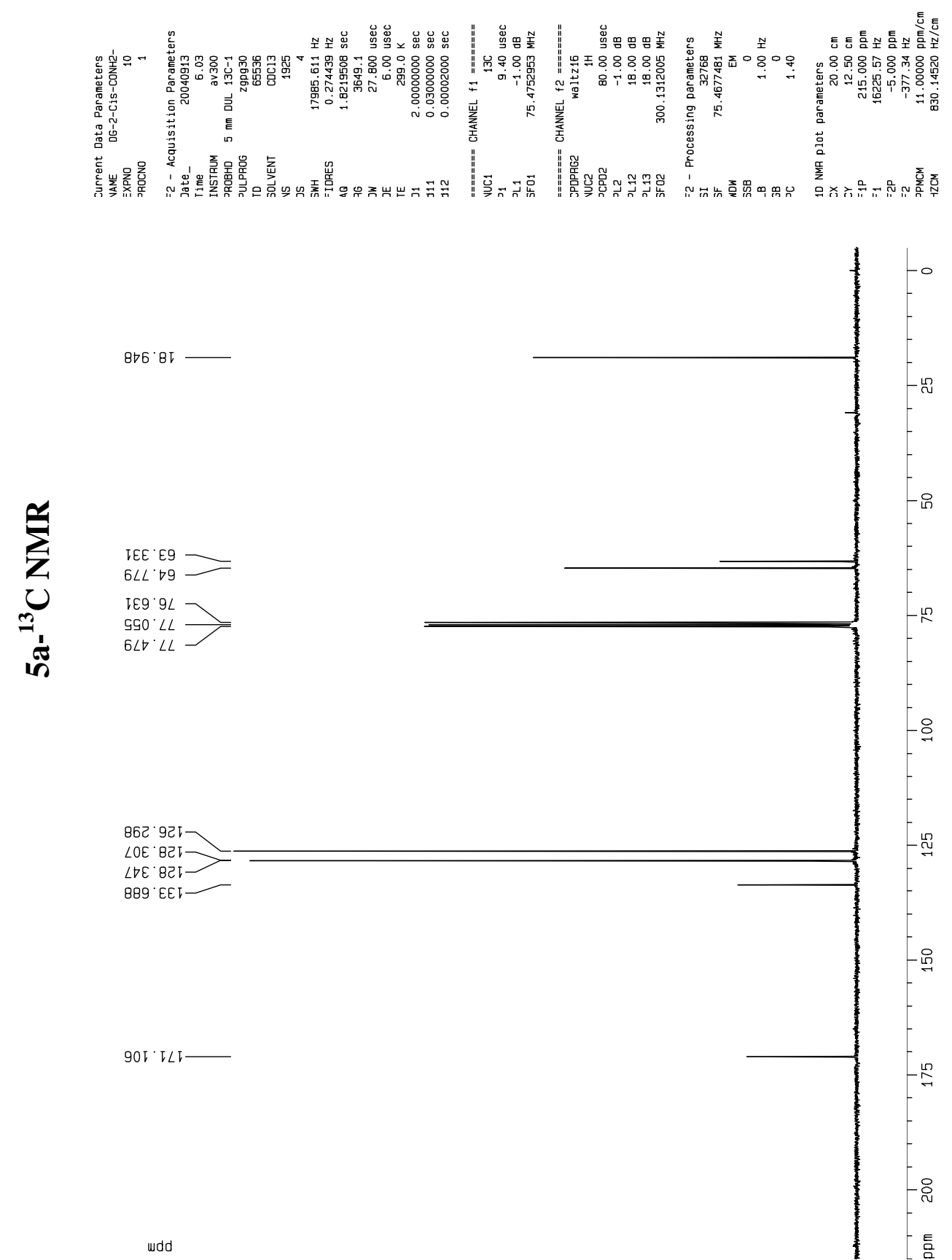

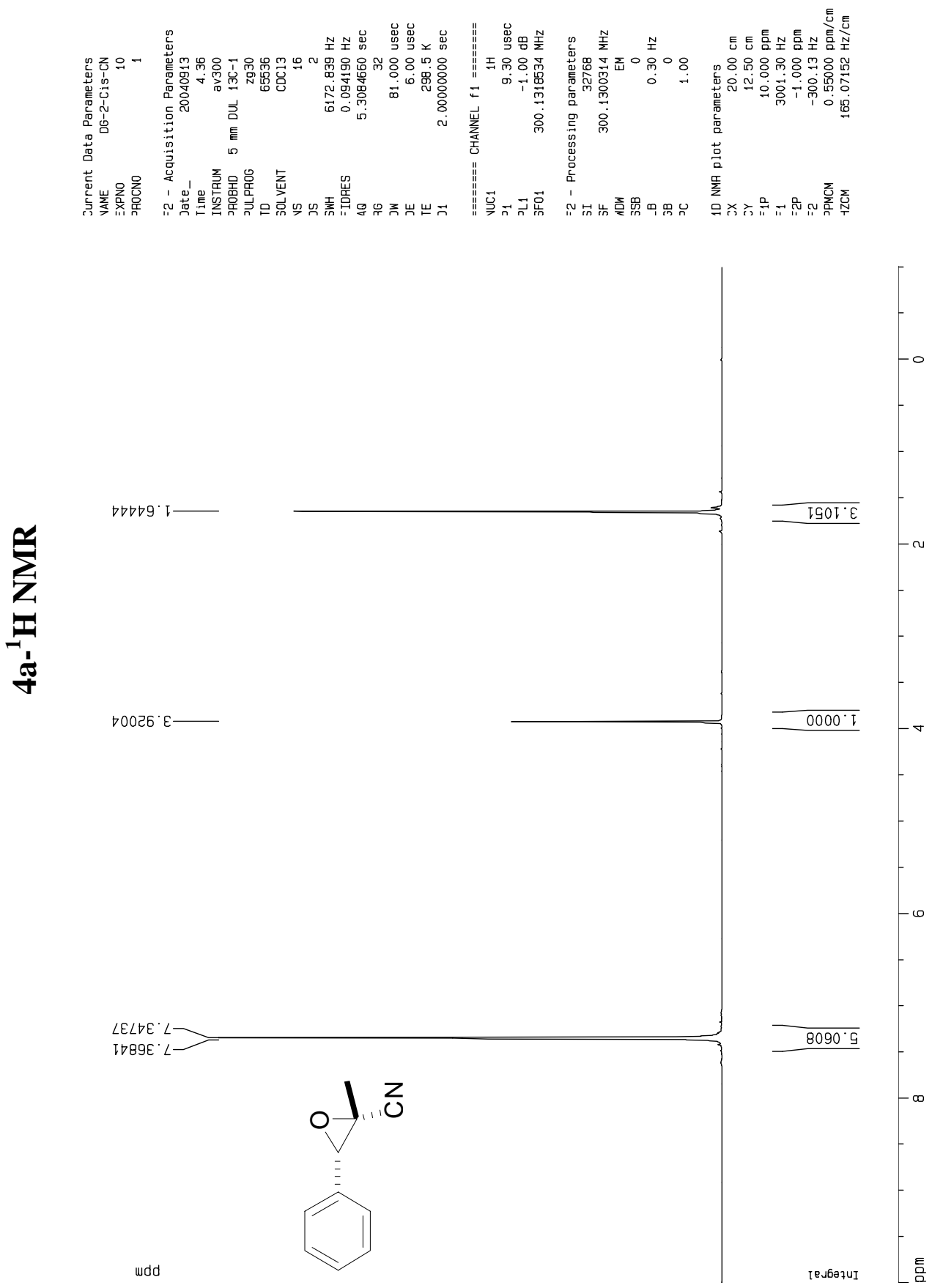


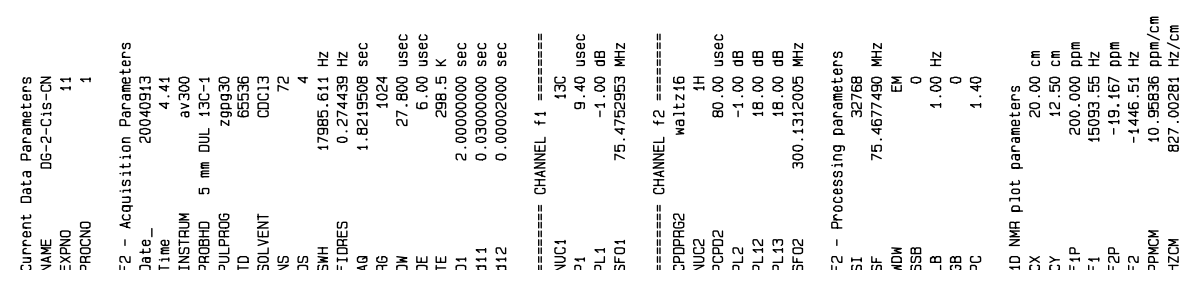

9E8.02

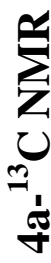

EI9'ES

EDT'D9 -

$2 \angle 6^{\circ} 9 L>$
$860^{\circ} \angle L$
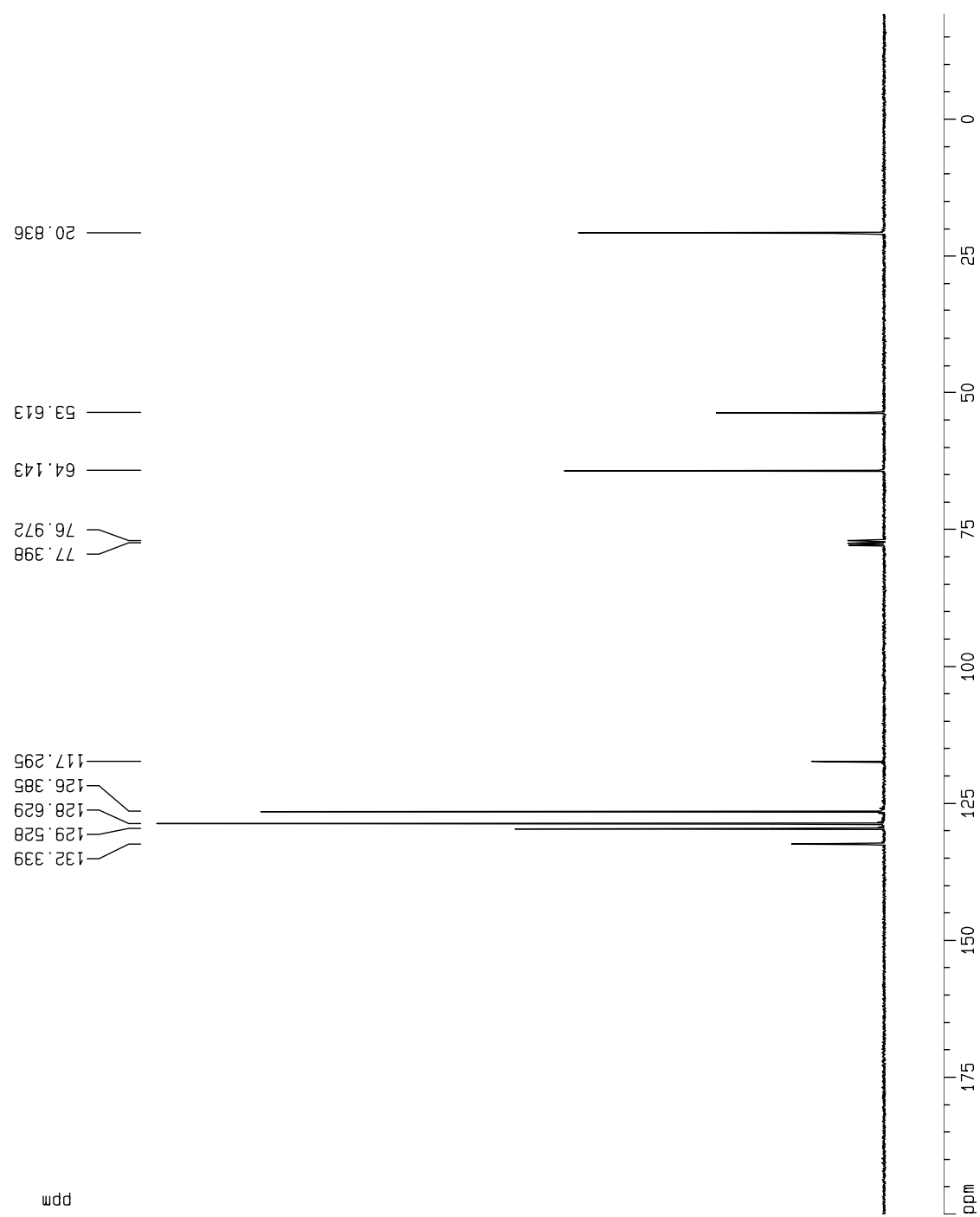

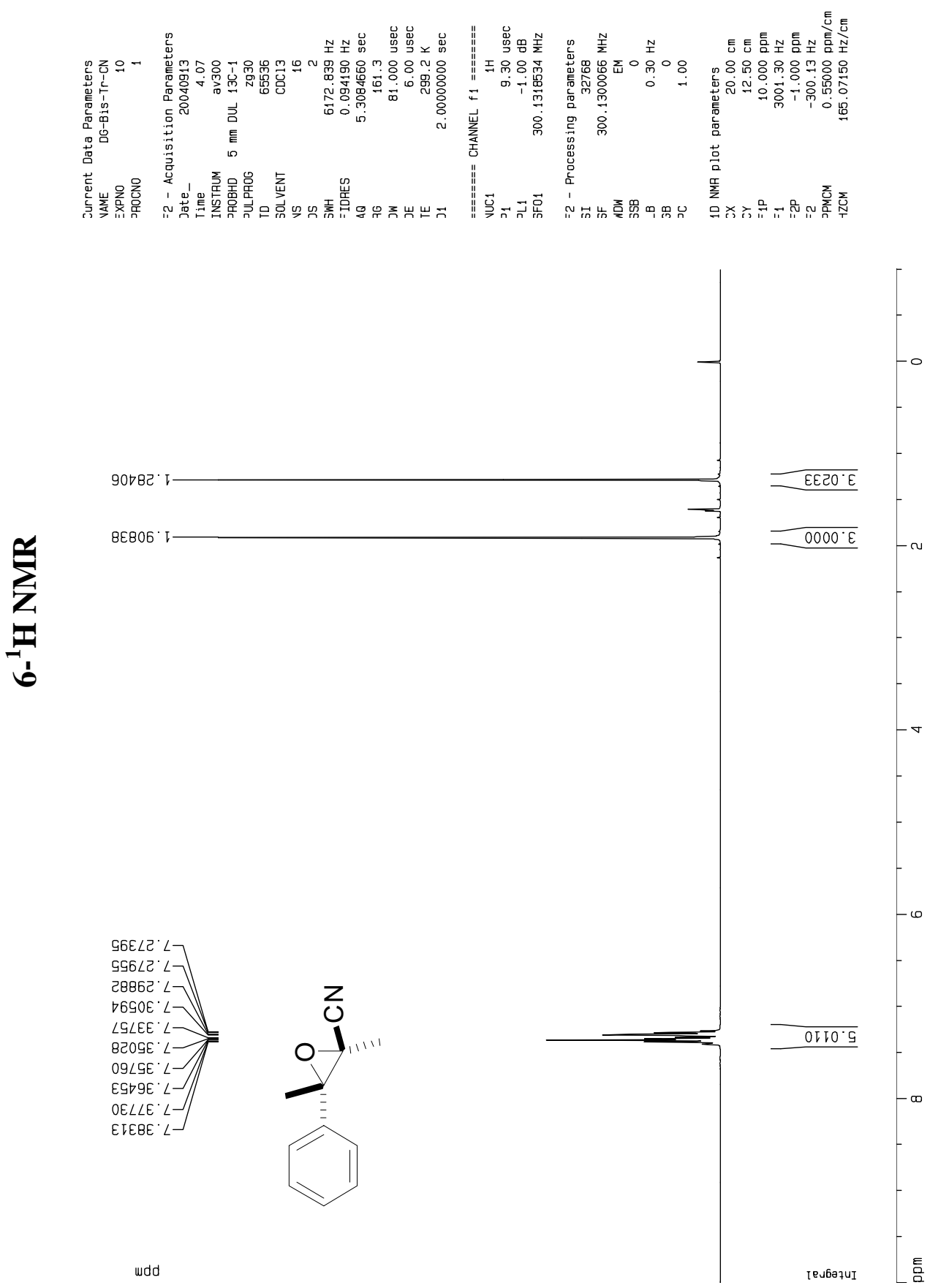

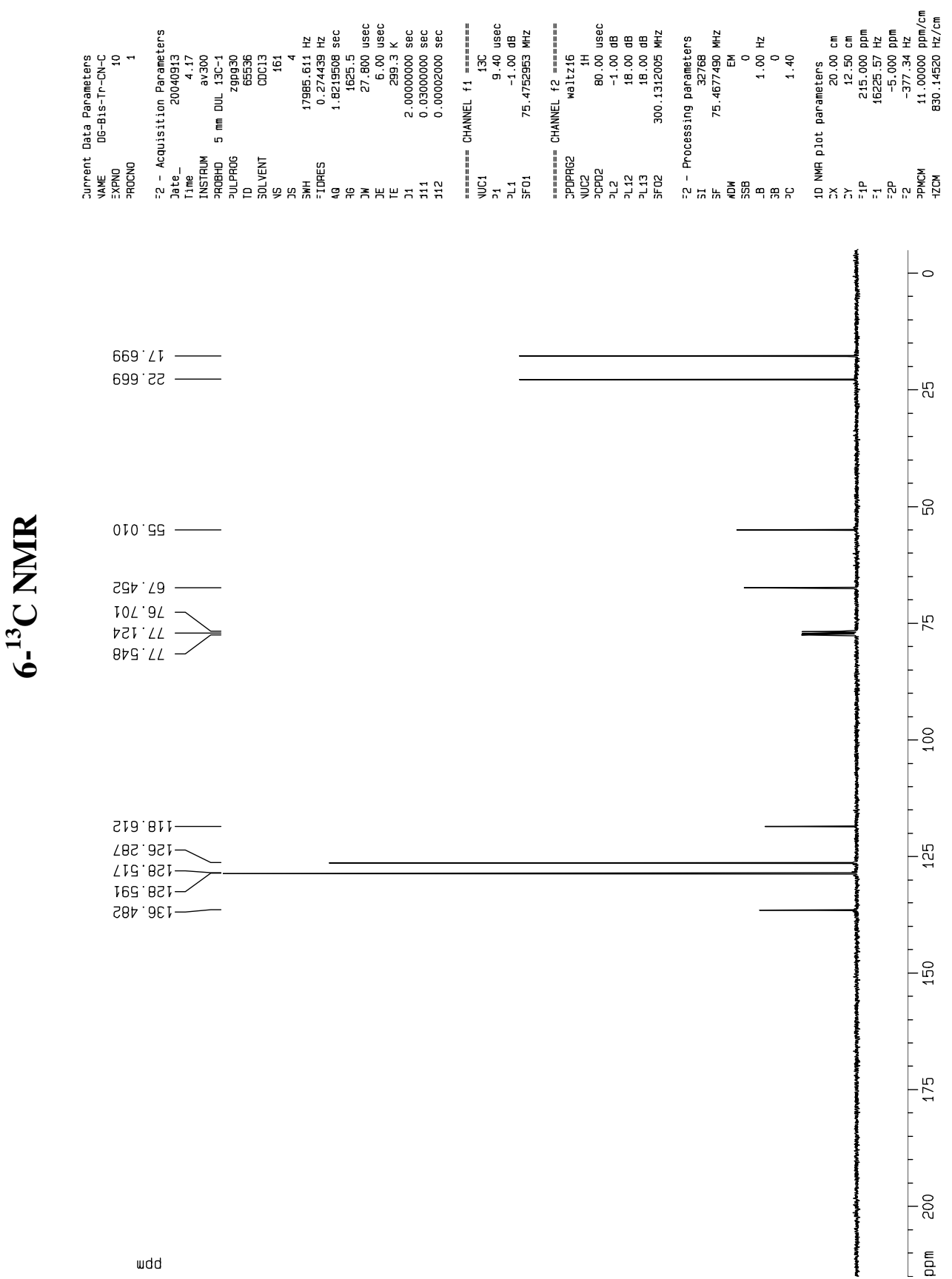

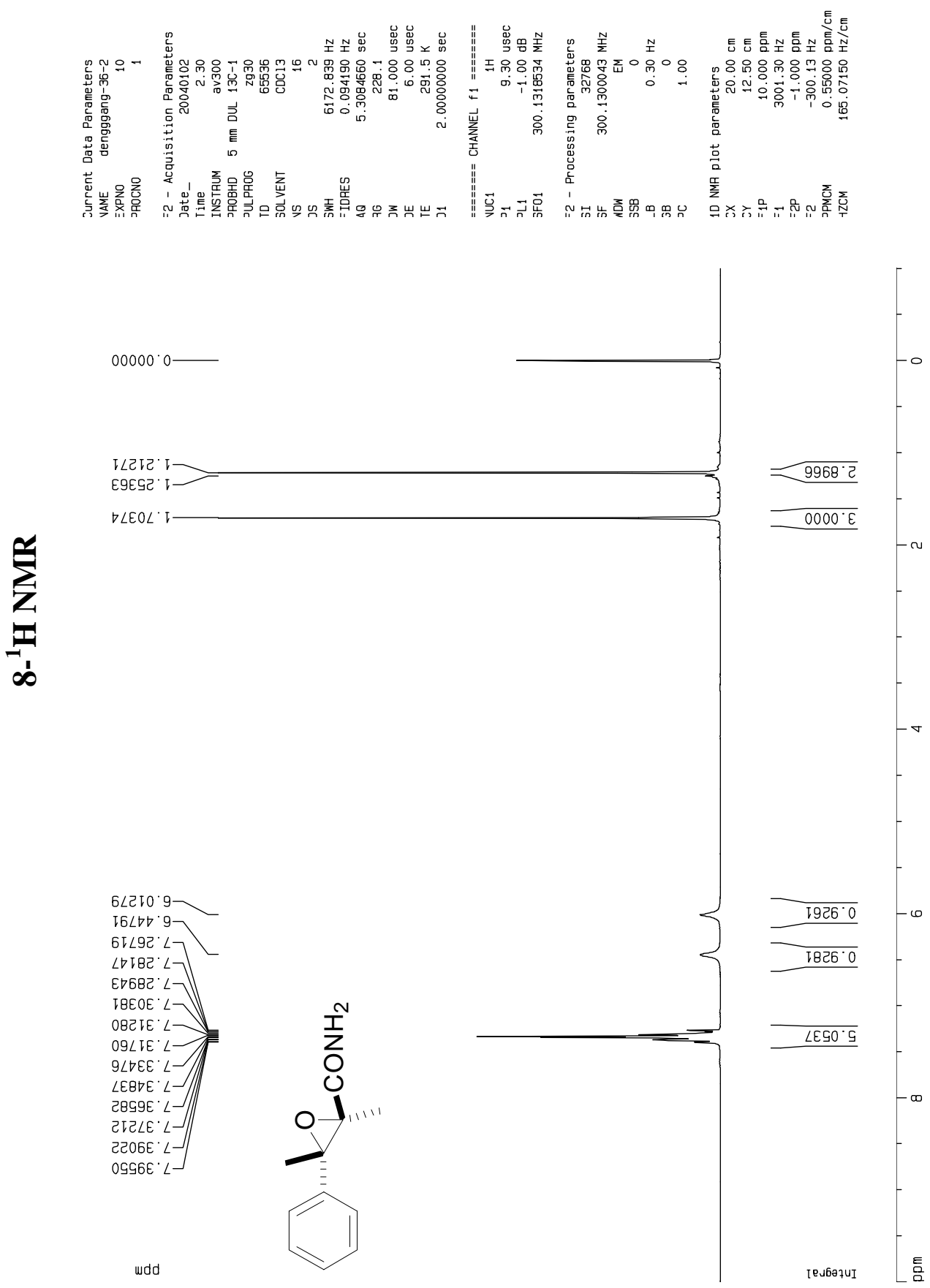

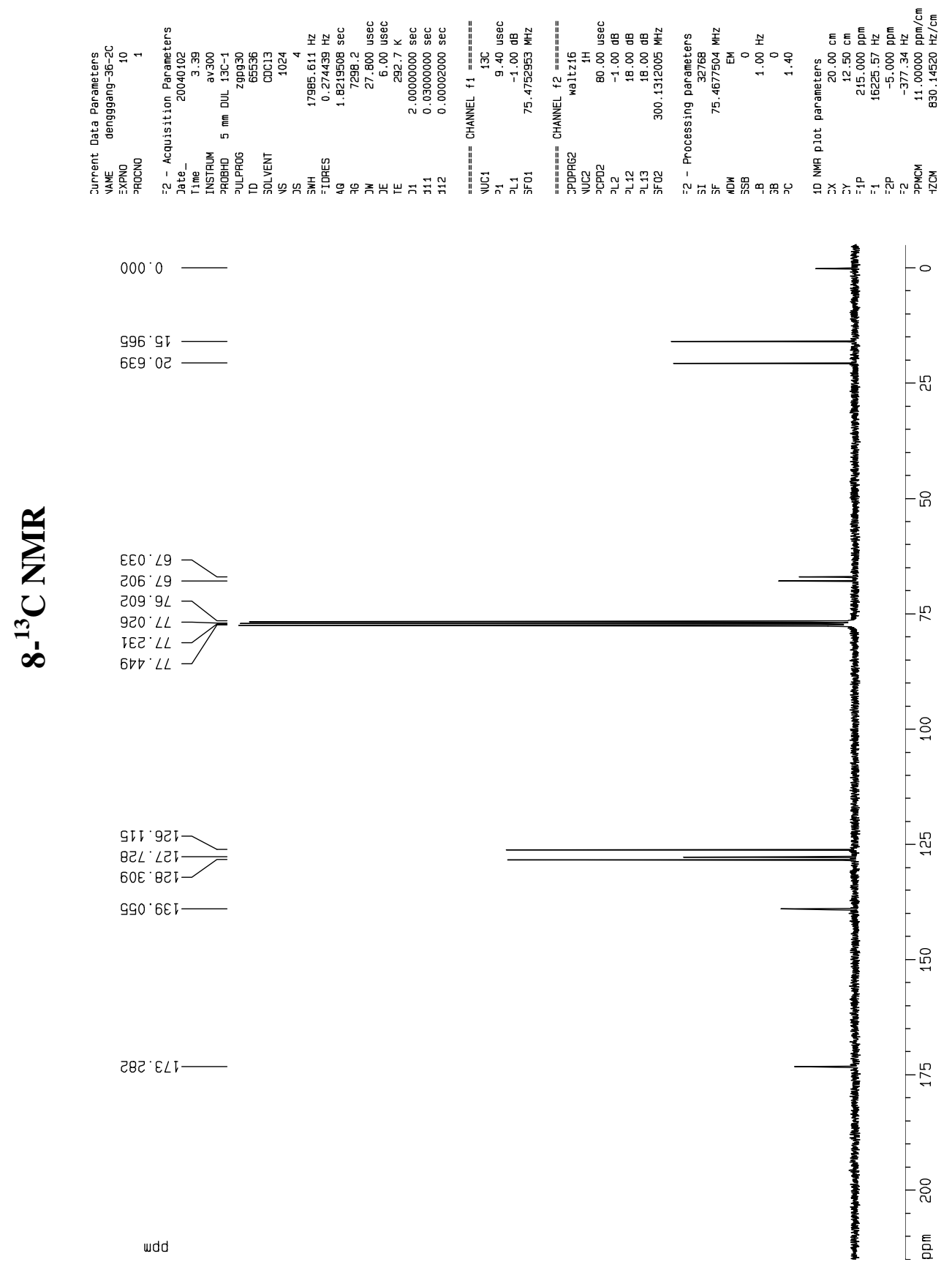

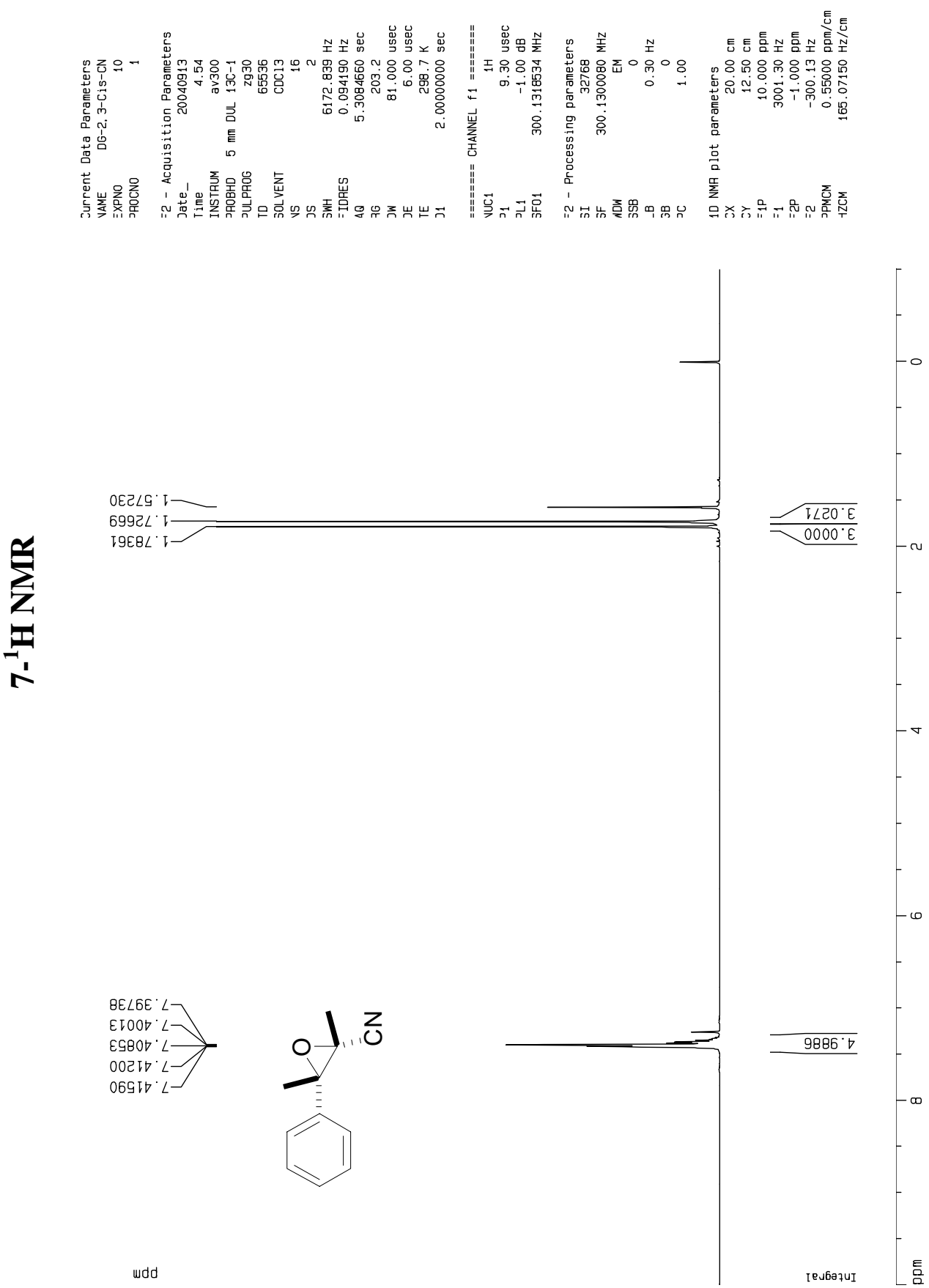


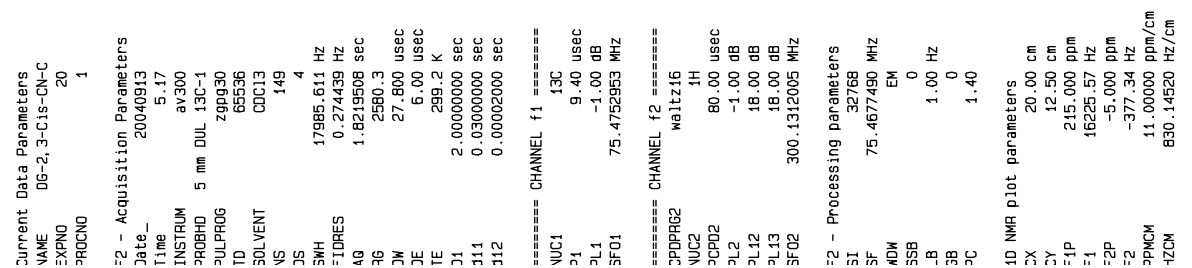

$\angle D \sigma^{\circ} \angle \tau$
$9 D \sigma^{\circ} \mathrm{BL}$
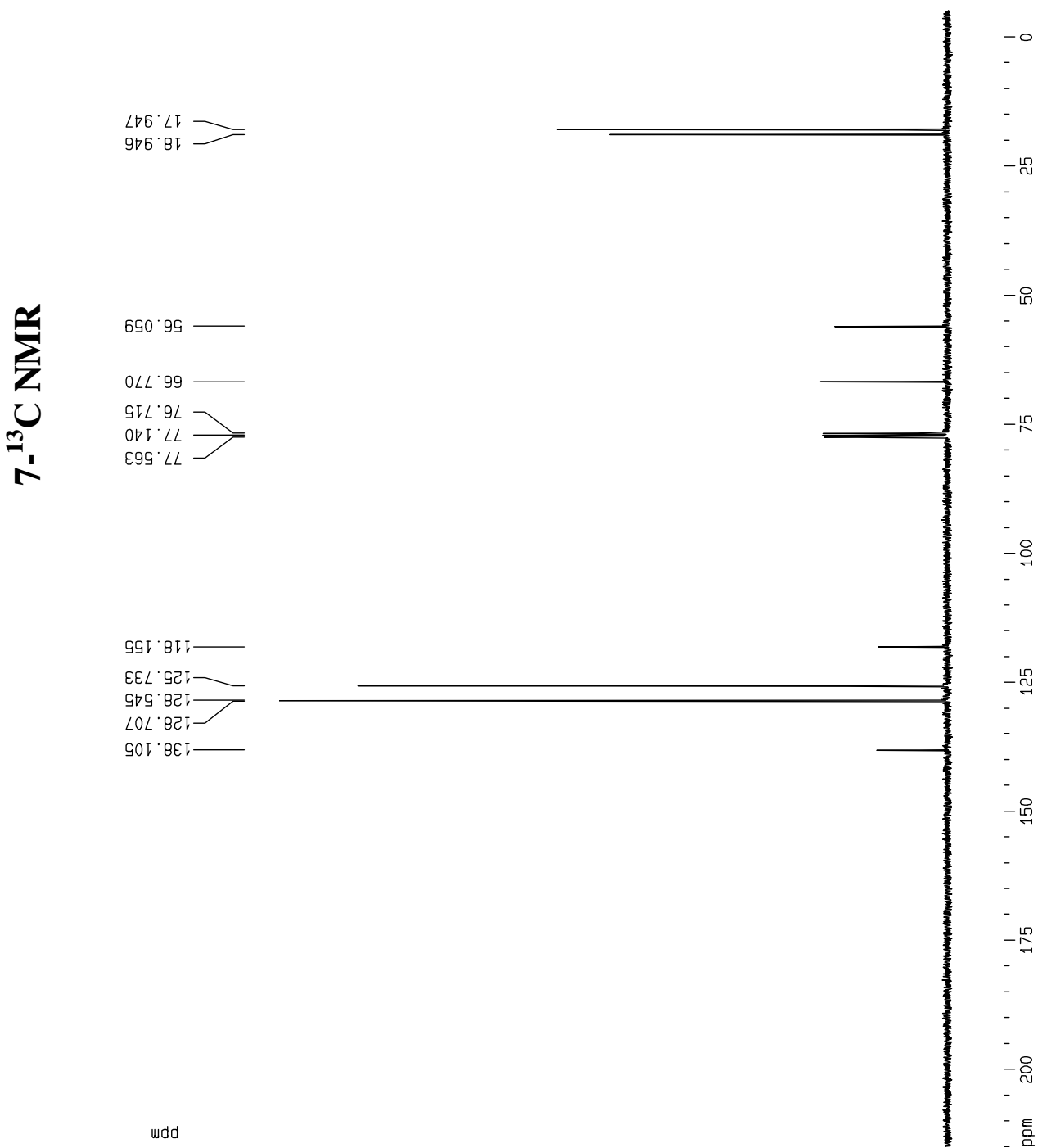

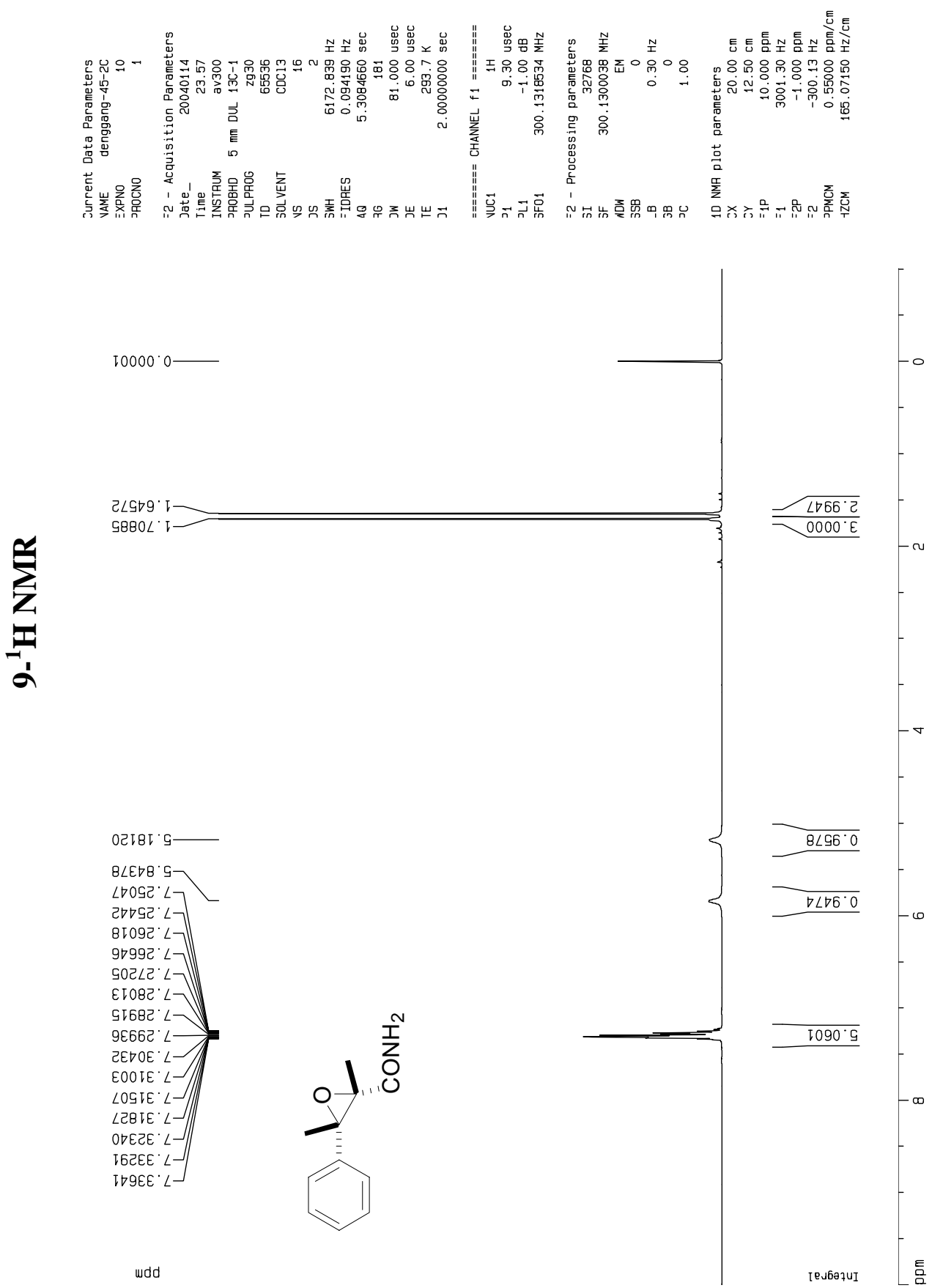

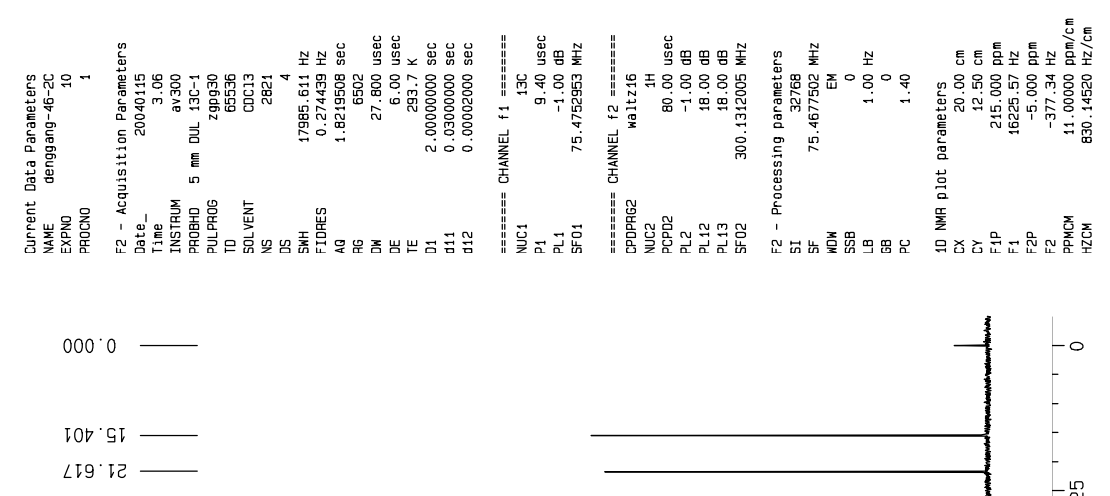

$\sum_{0}^{\infty}$

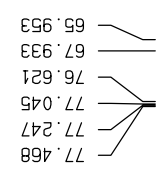

$\overline{ }$

$89 t^{\circ}<1$

S98.9टा一

$B 0 \angle \cdot \angle C I-$

E88. $\angle 21 工 \longrightarrow$

$680.851-$

$662 \cdot 821-$

LOG' $6 E \downarrow$

$909^{\circ} \mathrm{LI}$ 

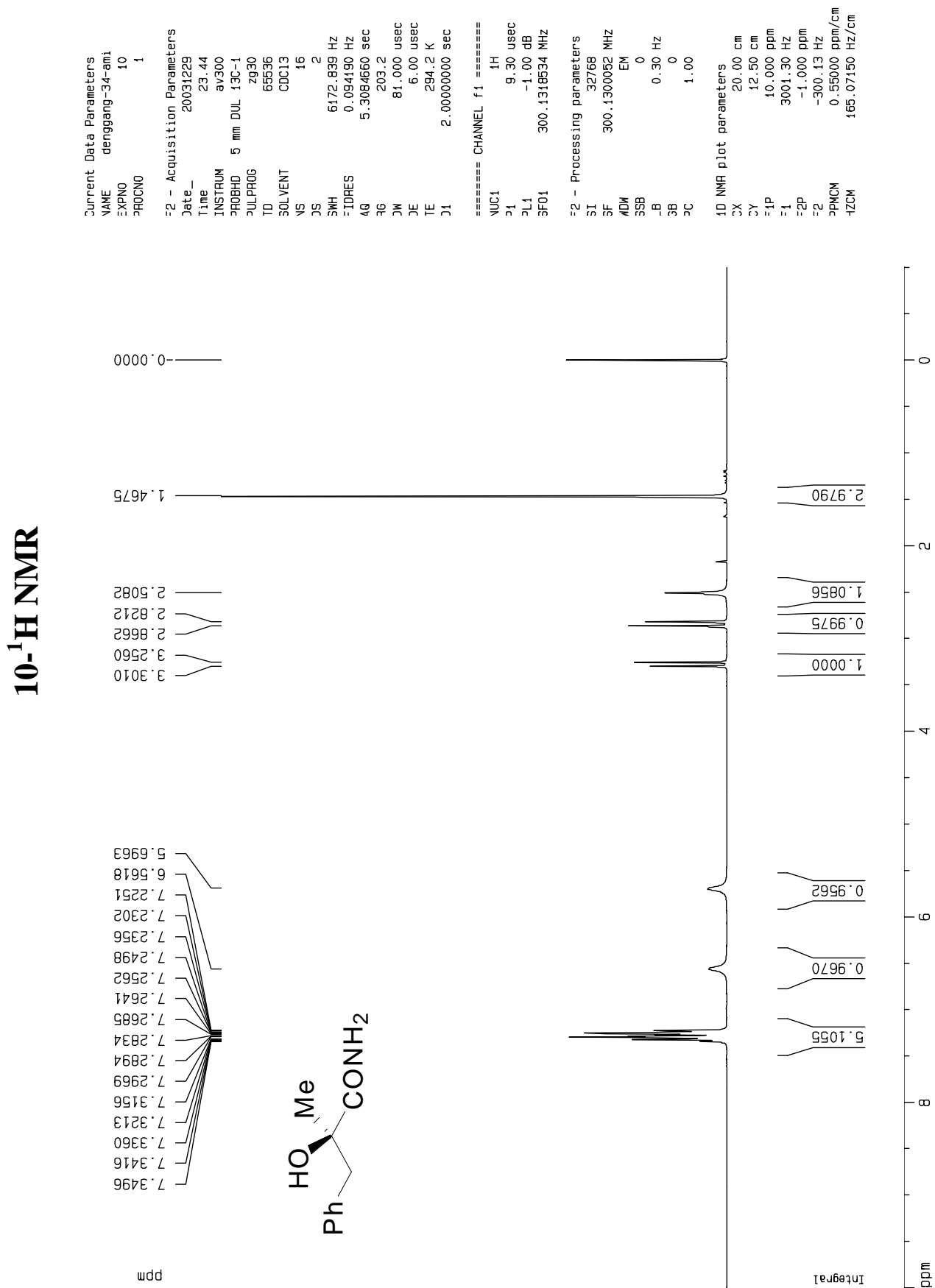

TevortuI 

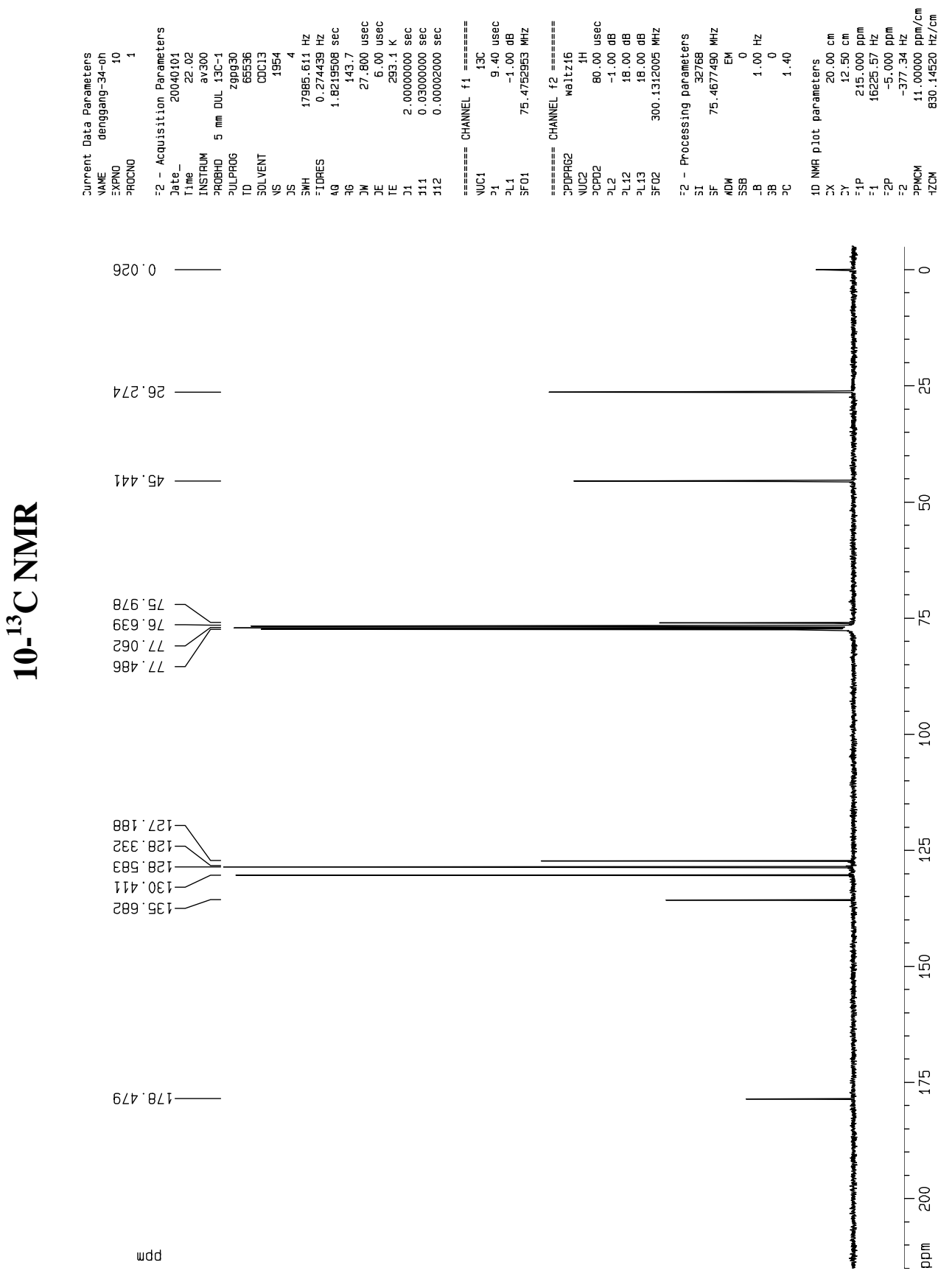

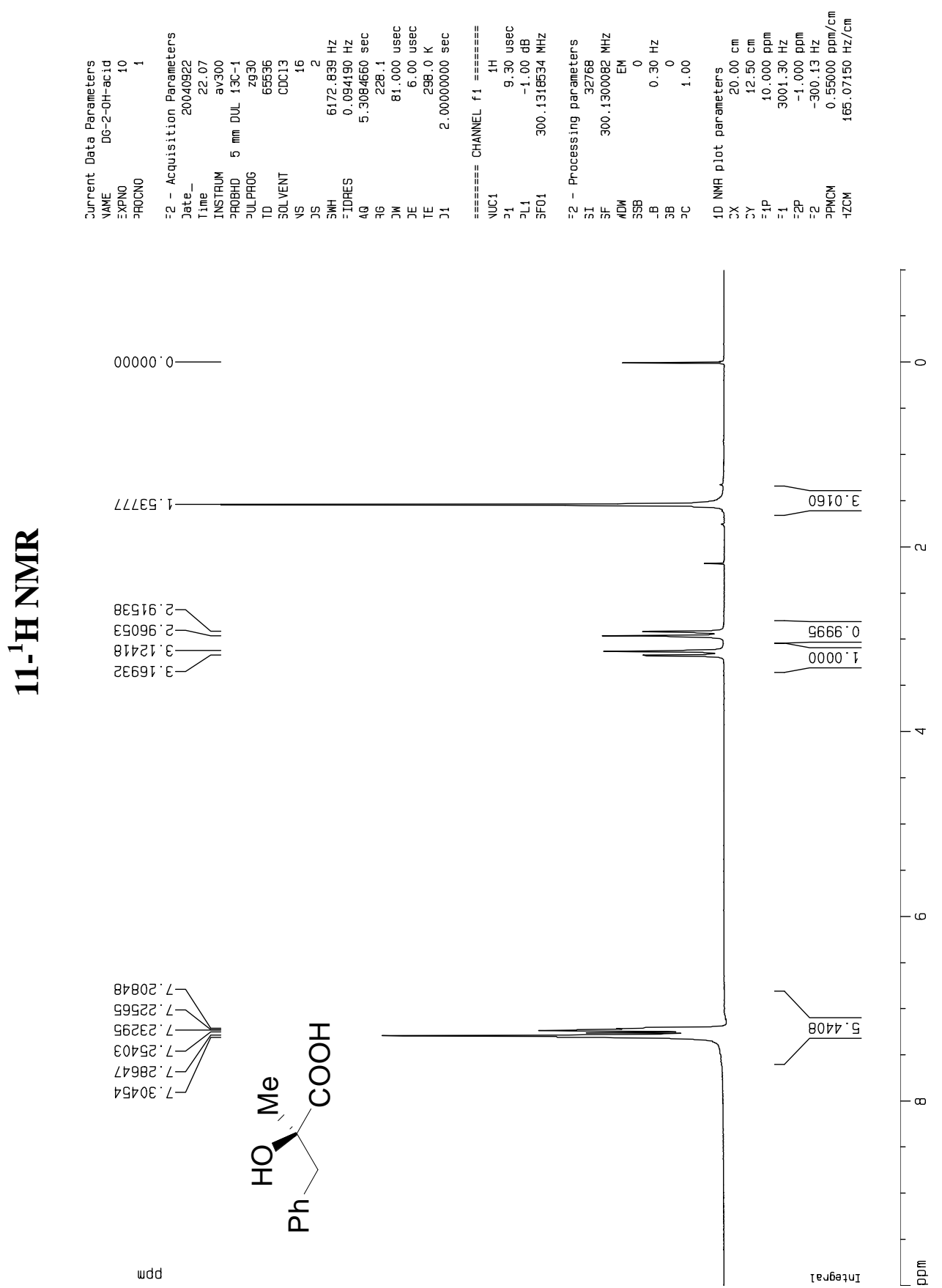

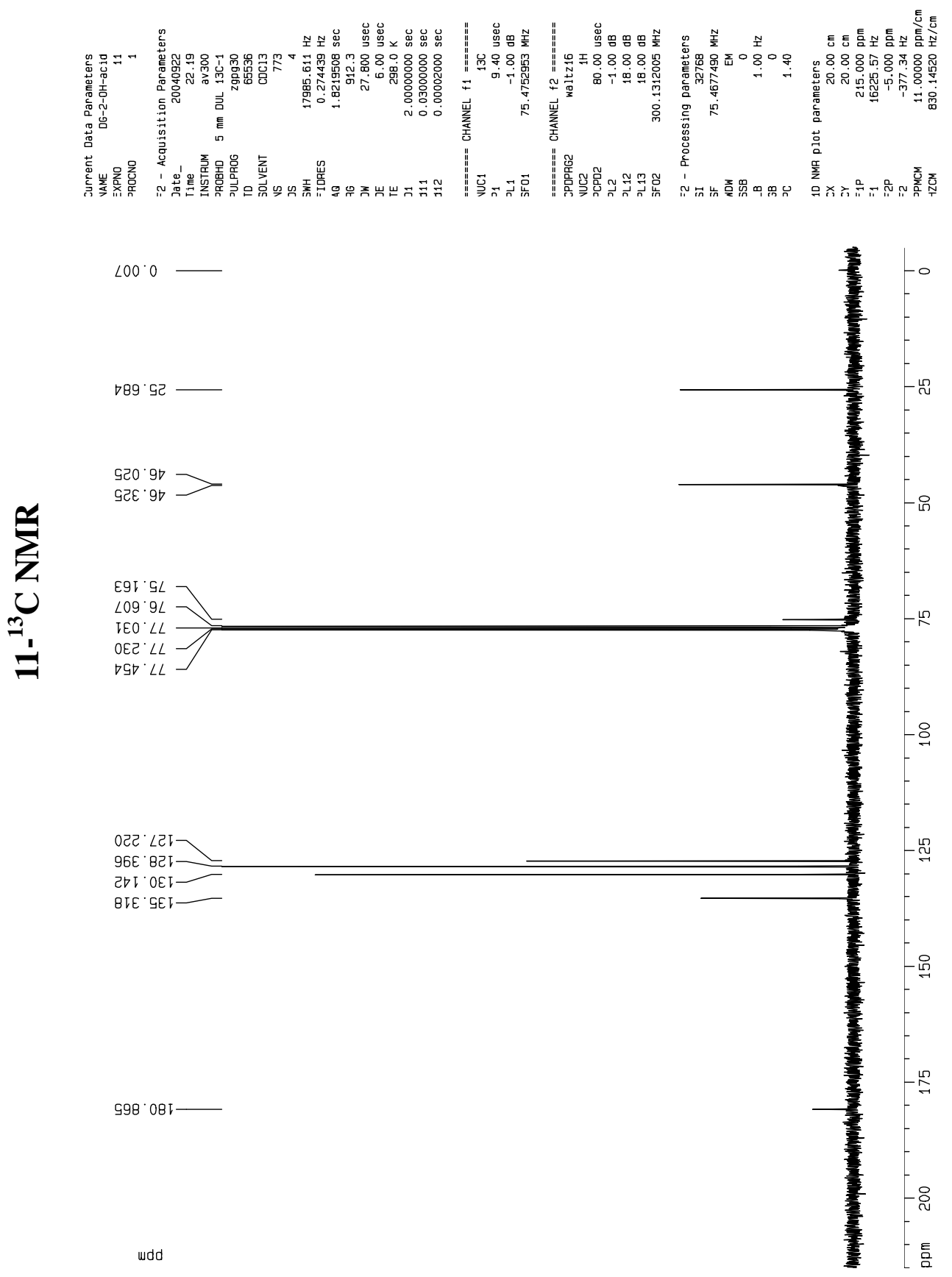

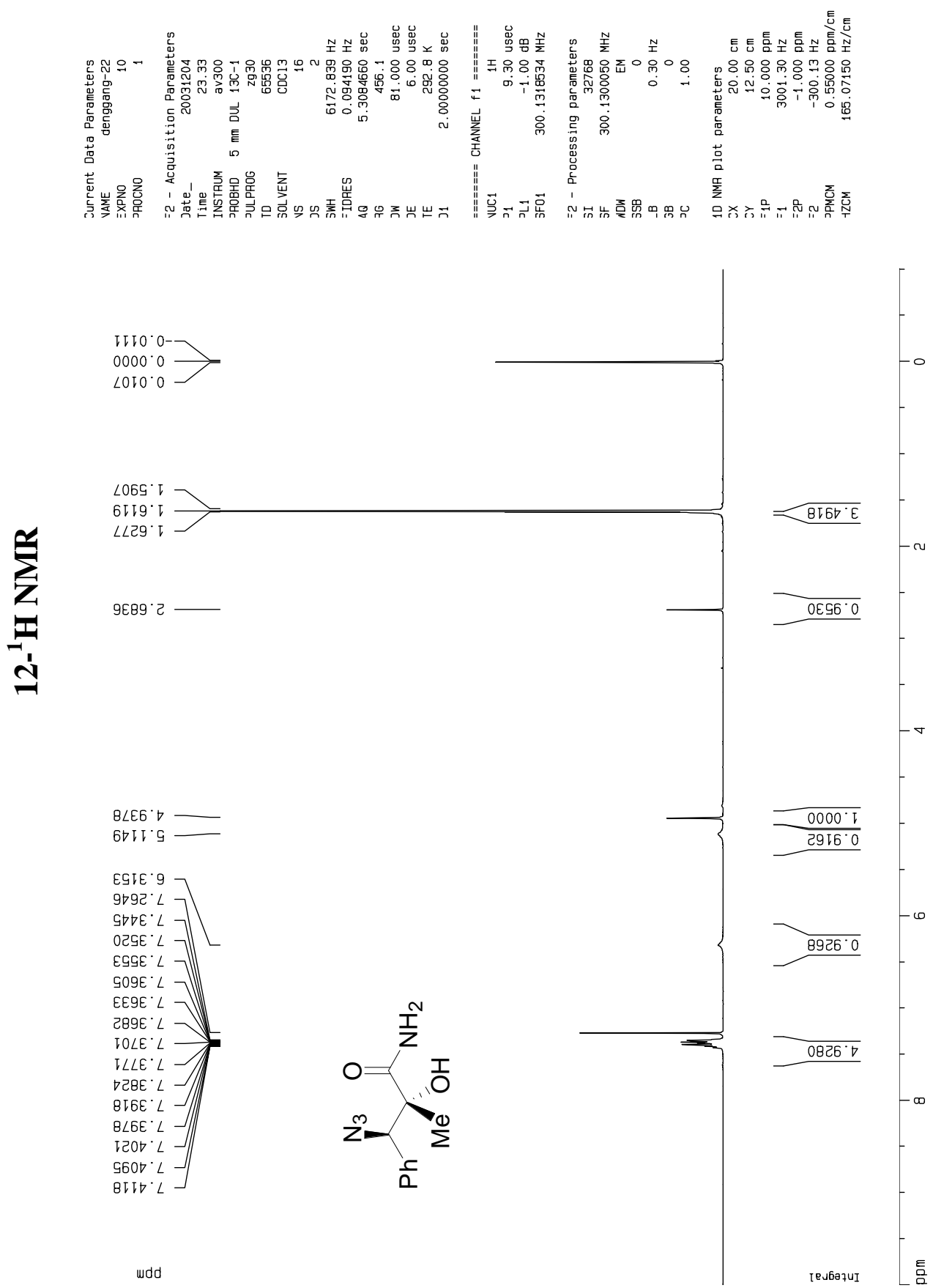

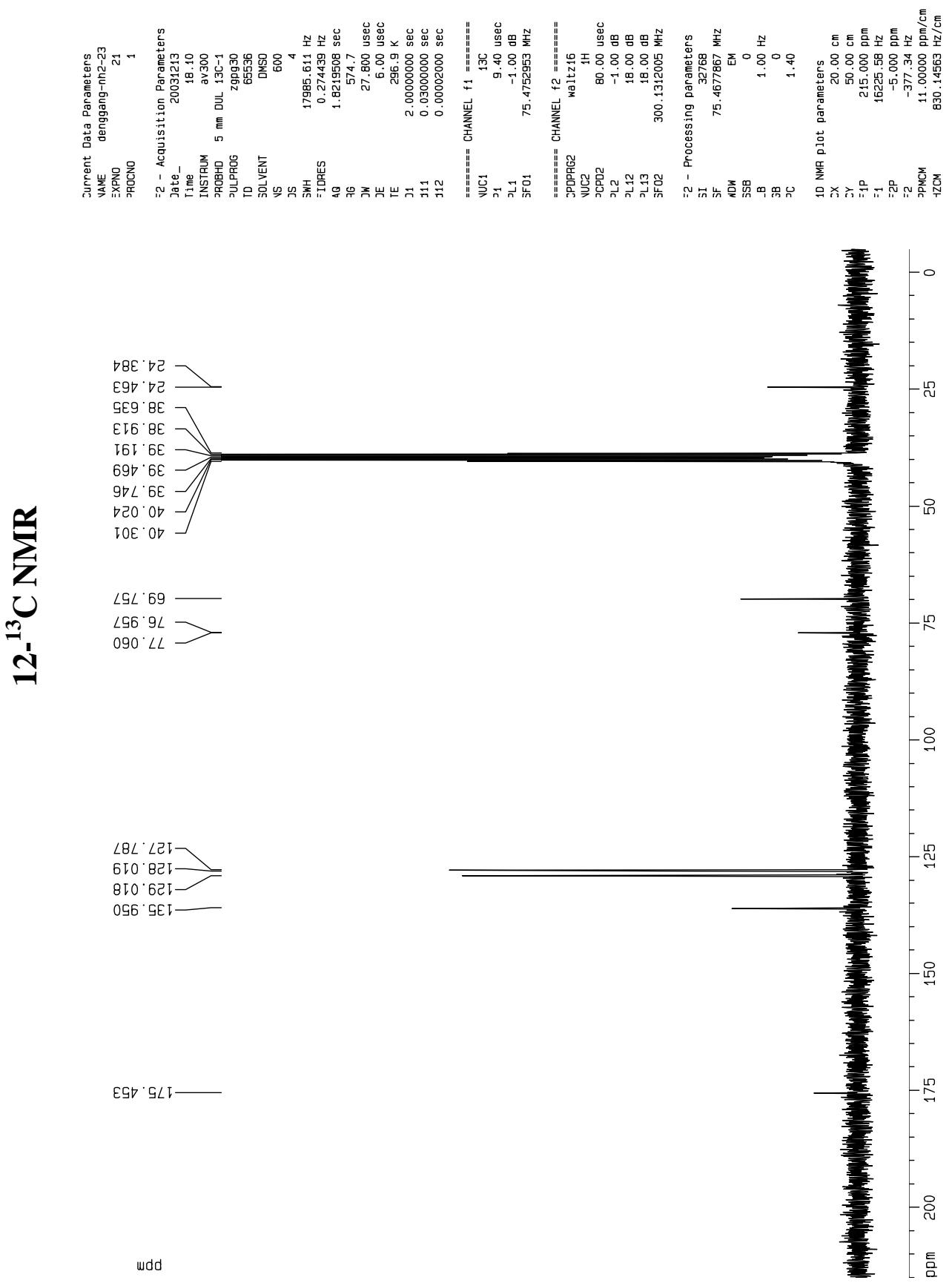

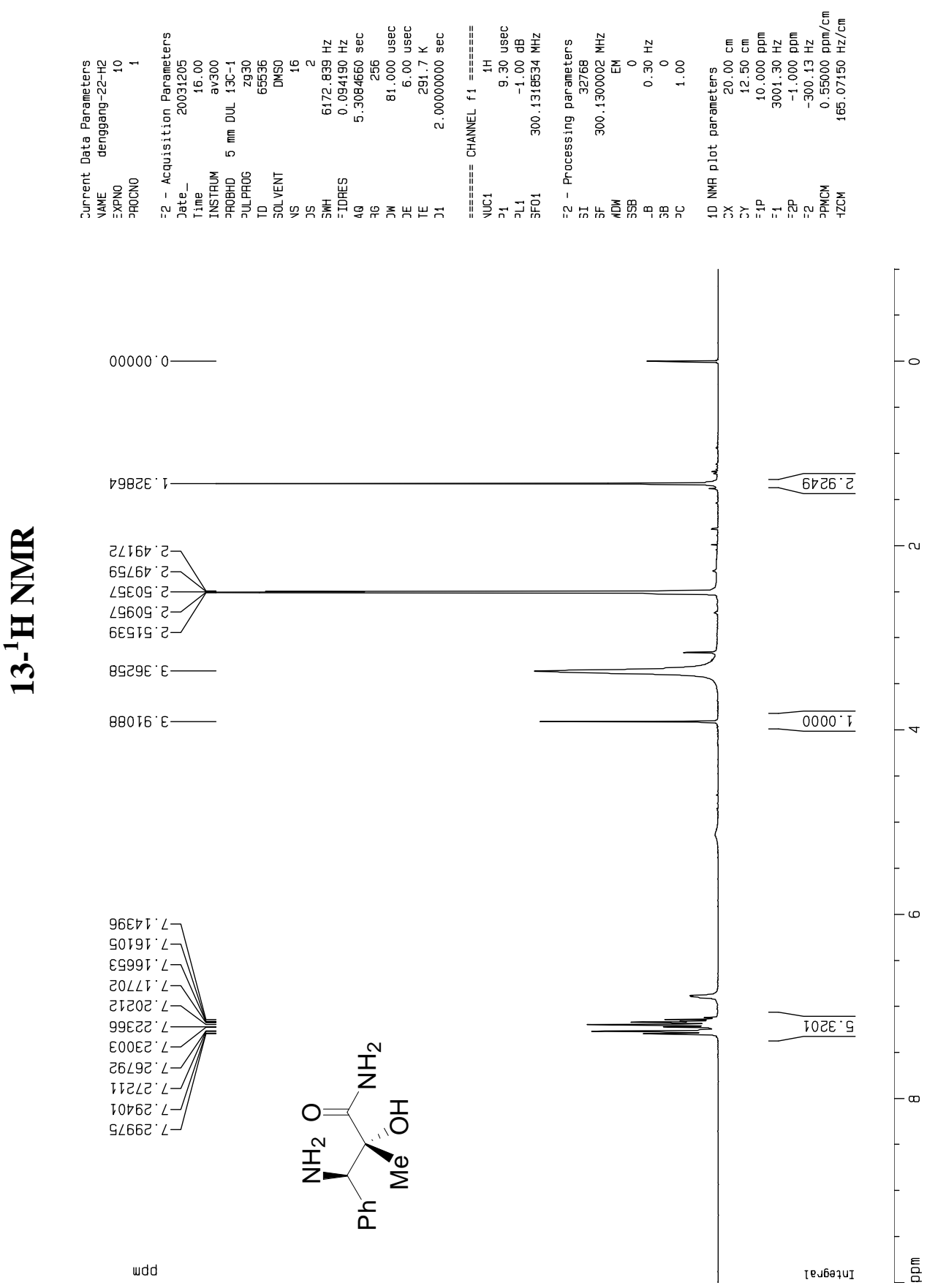

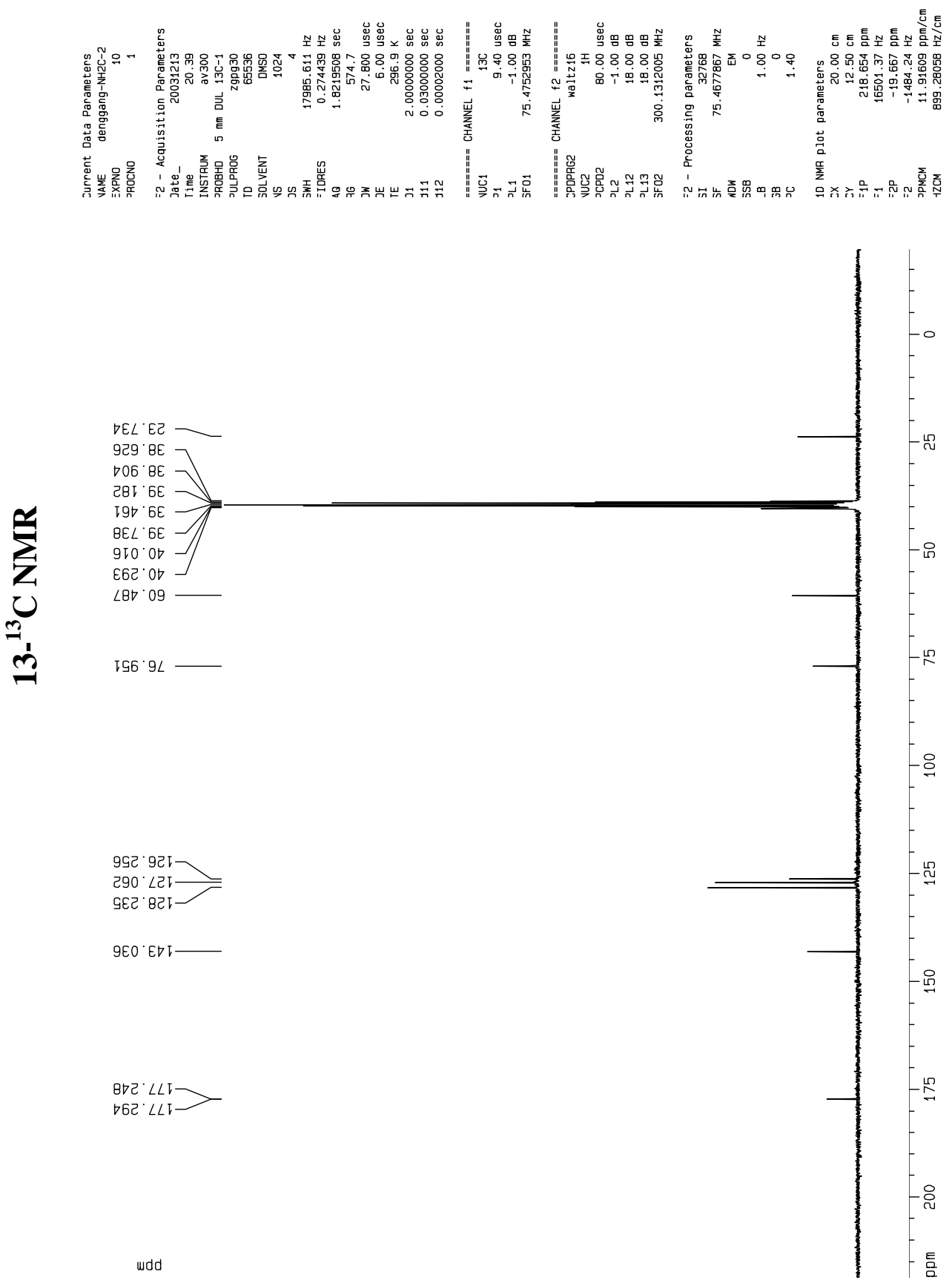

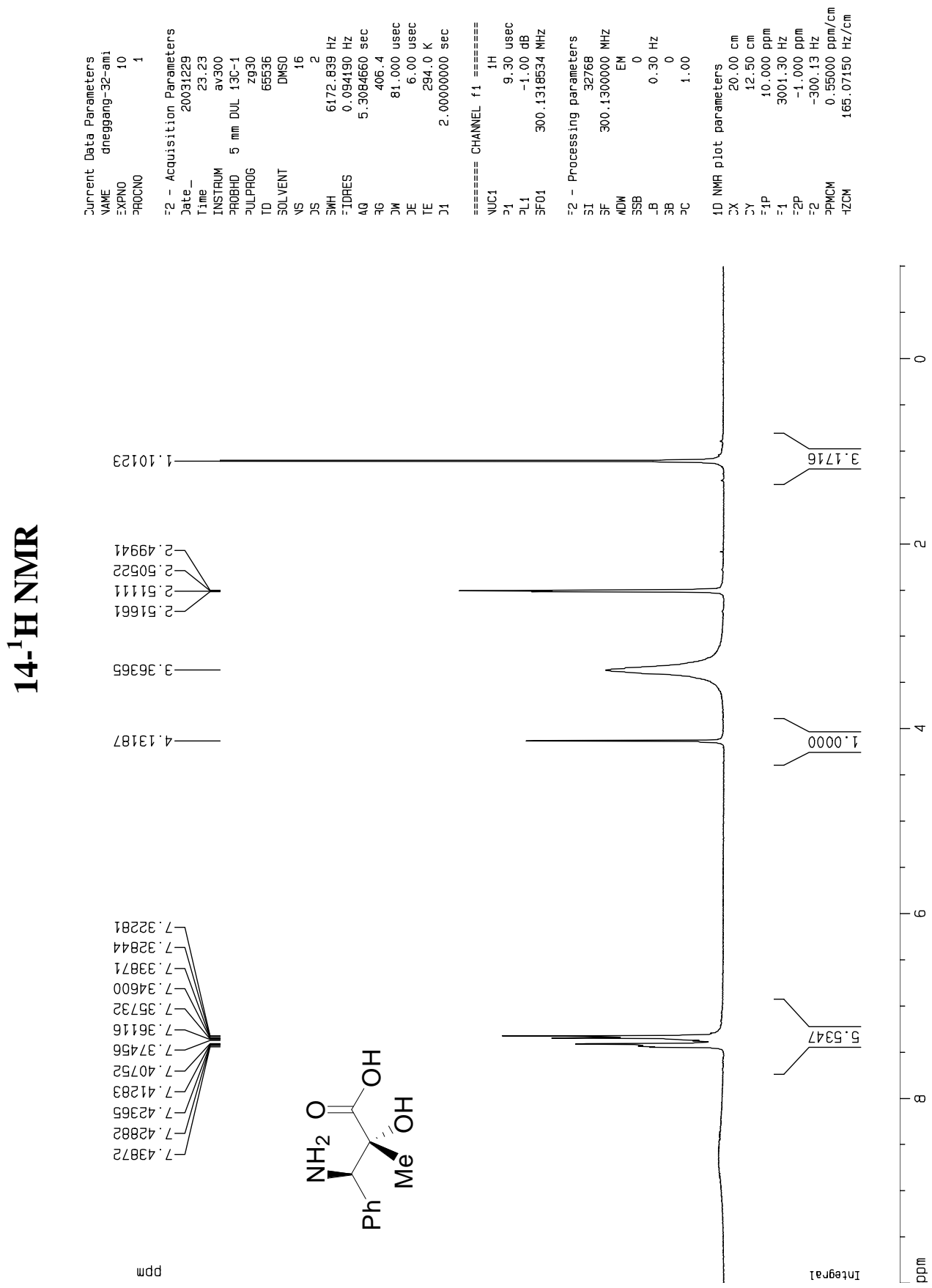

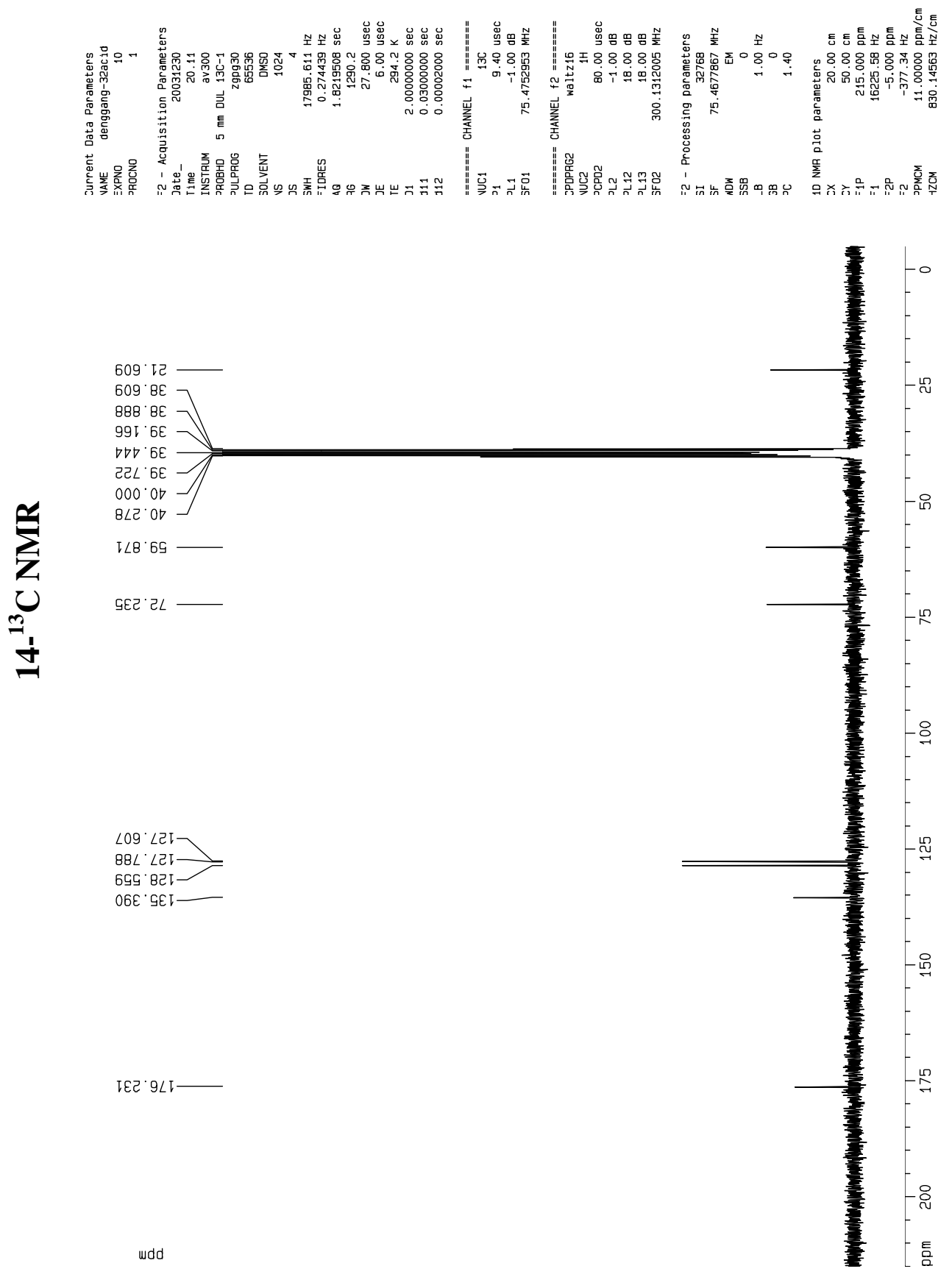

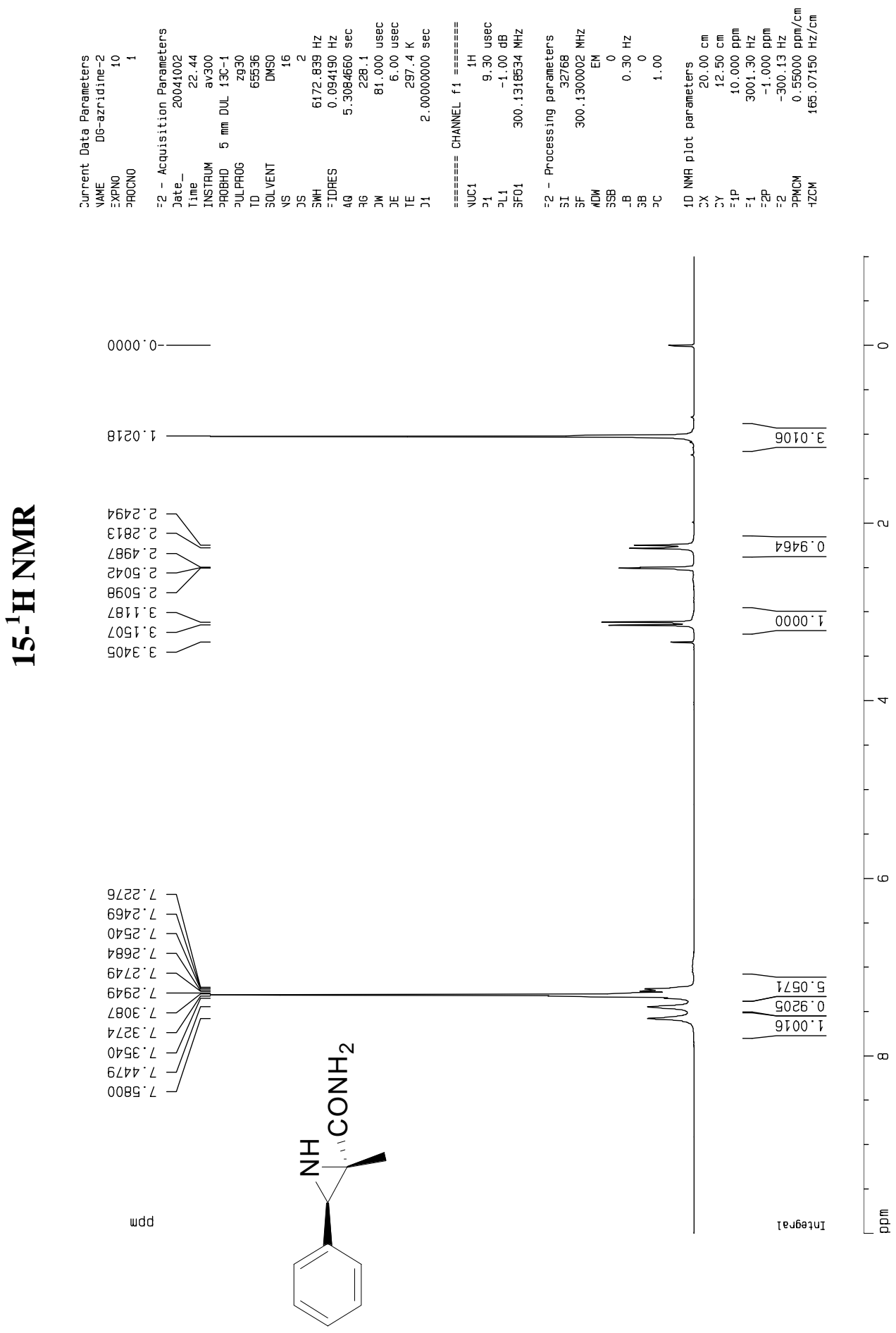

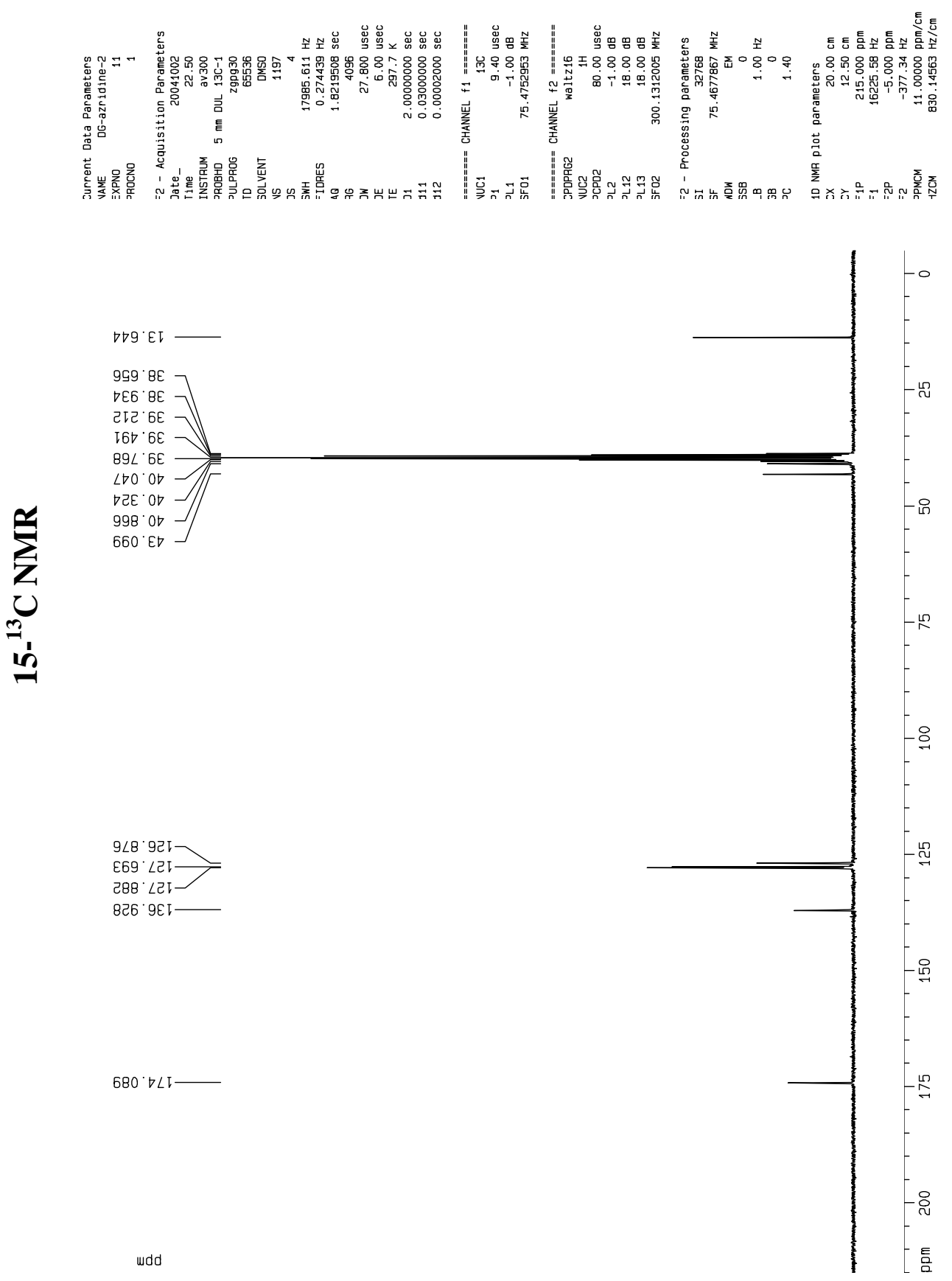


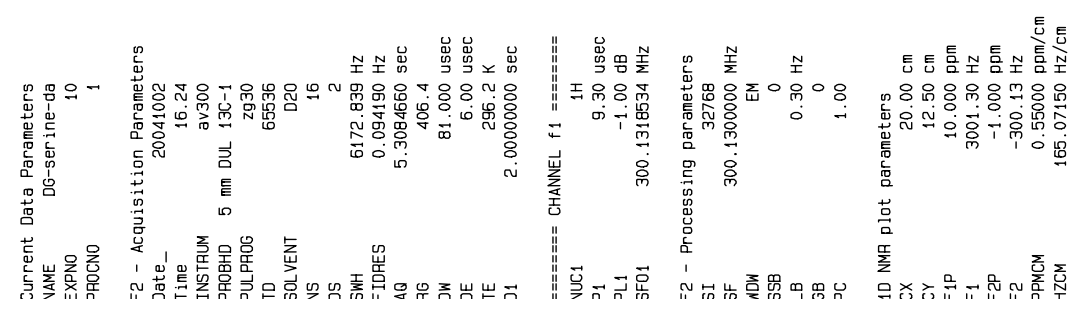

98029 ‘

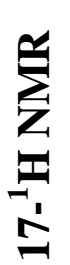

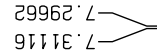

9ITE $L>$

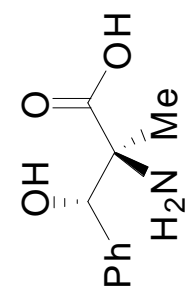

udd

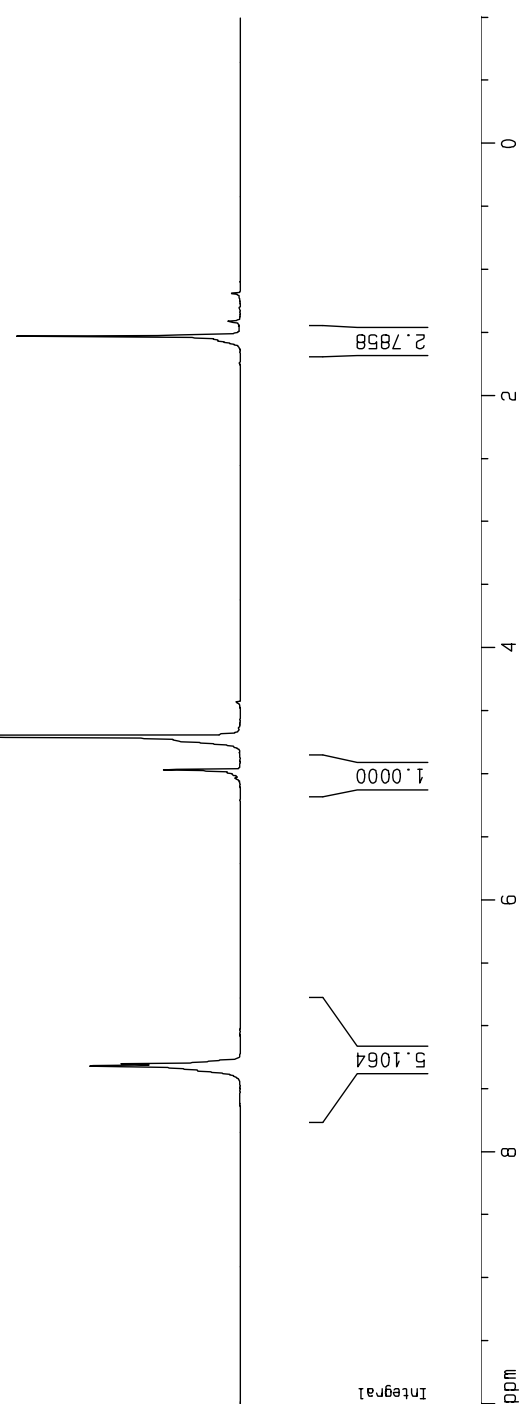



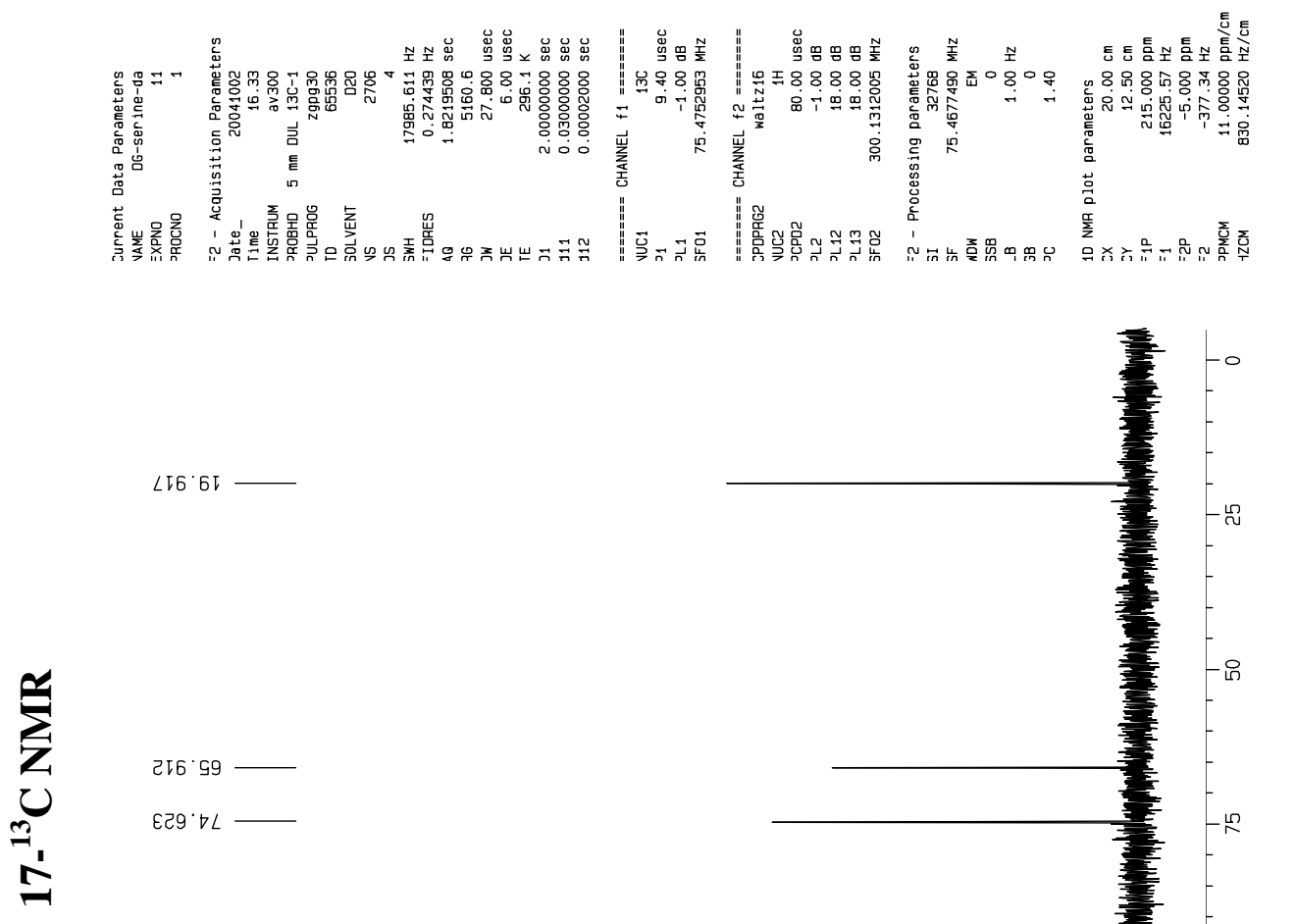

E $\angle 9.921 \longrightarrow$

टट9 $B ट 1-$

E9L $821-$

$68 D^{\circ} \angle E T-$

$\angle 9 C^{\circ} \nabla L I$

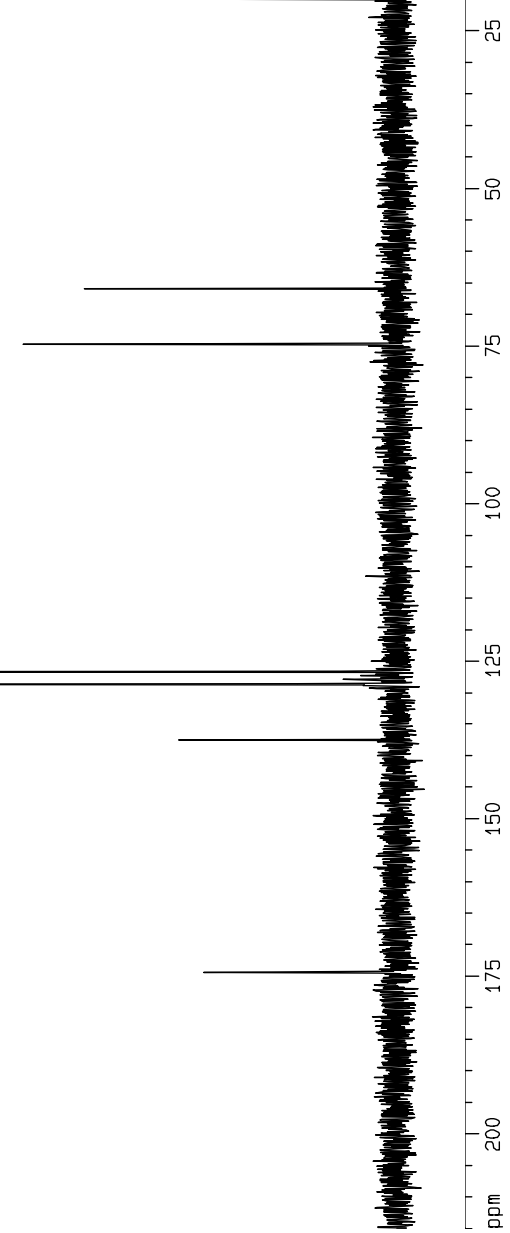




\section{HPLC analysis of products.}

A Shimadzu LC-10AVP HPLC system was used to analyze enantiomeric excess values of all products. A mixture of hexane:2-propanol [9:1], unless stated otherwise, as the mobile phase at a flow rate of $0.8 \mathrm{ml} / \mathrm{min}$ was employed.

Table S1. HPLC analysis of amides.

\begin{tabular}{|c|c|c|c|}
\hline Amide & $\boldsymbol{T}_{2 \boldsymbol{S}, \mathbf{B} \boldsymbol{R}}(\mathbf{m i n})$ & $\boldsymbol{T}_{\mathbf{2}, \mathbf{3 S}}(\mathbf{m i n})$ & Column \\
\hline $\mathbf{2 a}$ & 12.189 & 16.196 & Chiralcel OJ \\
\hline $\mathbf{2 b}$ & 12.720 & 17.904 & Chiralcel OD \\
\hline $\mathbf{2 c}$ & 11.698 & 17.957 & Chiralcel OD \\
\hline $\mathbf{2 d}$ & 12.659 & 17.406 & Chiralcel OD \\
\hline $\mathbf{2 e}$ & 10.558 & 12.304 & Chiralcel OD \\
\hline $\mathbf{2 f}$ & 13.817 & 21.028 & Chiralcel OD \\
\hline $\mathbf{2 g}$ & 11.401 & 14.881 & Chiralcel OD \\
\hline $\mathbf{2 h}$ & 9.985 & 11.267 & Chiralcel OJ \\
\hline $\mathbf{2} \mathbf{5}$ & 18.824 & 26.253 & Chiralcel OD \\
\hline $\mathbf{5 a}$ & 11.286 & 13.039 & Chiralcel OD \\
\hline $\mathbf{8}$ & 7.804 & 9.539 & Chiralcel OJ \\
\hline $\mathbf{9}$ & 7.585 & 8.916 & Chiralcel OJ \\
\hline
\end{tabular}

Mobile phase: hexane : isopropanol $=9: 1$; flow rate $0.8 \mathrm{ml} / \mathrm{min} ; 25\llcorner$

Table S2. HPLC analysis of nitriles

\begin{tabular}{|c|c|c|c|}
\hline Nitrile & $\boldsymbol{T}_{\boldsymbol{I}}(\mathbf{m i n})$ & $\boldsymbol{T}_{\mathbf{2}}(\mathbf{m i n})$ & Column \\
\hline $\mathbf{1 a}$ & 8.605 & 9.666 & Chiralcel OJ \\
\hline $\mathbf{4 a}$ & 14.427 & 16.957 & Chiralcel OJ \\
\hline $\mathbf{4 e}$ & 7.575 & 9.323 & Chiralcel OB \\
\hline $\mathbf{6}$ & 7.110 & 7.986 & Chiralcel OJ \\
\hline $\mathbf{7}$ & 11.355 & 16.396 & Chiralcel OJ \\
\hline
\end{tabular}

Mobile phase: hexane:iso-propanol=9:1; flow rate $0.8 \mathrm{ml} / \mathrm{min} ; 25\llcorner$

Table S3. HPLC analysis of the ring opening products

\begin{tabular}{|c|c|c|c|}
\hline Compound & $\boldsymbol{T}_{\boldsymbol{1}}(\mathbf{m i n})$ & $\boldsymbol{T}_{\mathbf{2}}(\mathbf{m i n})$ & Column \\
\hline $\mathbf{1 0}$ & $7.15(\mathrm{R})$ & $8.63(\mathrm{~S})$ & AD \\
\hline $\mathbf{1 1}$ & $12.03(\mathrm{R})$ & $17.86(\mathrm{~S})$ & AD \\
\hline $\mathbf{1 2}$ & $16.72(2 \mathrm{R}, 3 \mathrm{R})$ & $21.74(2 \mathrm{~S}, 3 \mathrm{~S})$ & OJ \\
\hline $\mathbf{1 3}^{\mathrm{a}}$ & $17.43(2 \mathrm{~S}, 3 \mathrm{~S})$ & $22.94(2 \mathrm{R}, 3 \mathrm{R})$ & OD \\
\hline $\mathbf{1 4}^{\mathrm{b}}$ & $13.42(2 \mathrm{~S}, 3 \mathrm{~S})$ & $17.22(2 \mathrm{R}, 3 \mathrm{R})$ & ${\text { Chirobiotic } \mathrm{TAG}^{\mathrm{TM}}}^{\mathrm{T}}$ \\
\hline $\mathbf{1 7}^{\mathrm{c}}$ & $24.76(2 \mathrm{R}, 3 \mathrm{R})$ & $26.80(2 \mathrm{~S}, 3 \mathrm{~S})$ & Chirobiotic $^{\mathrm{c}} \mathrm{TAG}^{\mathrm{TM}}$ \\
\hline
\end{tabular}


Mobile phase: hexane $:$ isopropanol $=9: 1$; flow rate $0.8 \mathrm{ml} / \mathrm{min} ; 25\llcorner$

a': Mobile phase: hexane : isopropanol $=9: 1\left(0.1 \% \mathrm{Et}_{2} \mathrm{NH}\right)$.

${ }^{\mathrm{b}}$ : Mobile phase: methanol ( $1 \%$ triethylamine and $1 \%$ acetic acid), $30\llcorner$.

${ }^{C}$ : Mobile phase: Methanol : $\mathrm{H}_{2} \mathrm{O}=6: 4$, flow rate $0.2 \mathrm{ml} / \mathrm{min}, 20$ L 
